\title{
Mineração de Dados aplicada à tuberculose nos municípios do estado de São Paulo
}

\author{
Erick Pereira Santos
}

\author{
DisSERTAÇÃO APRESENTADA \\ $\mathrm{AO}$ \\ Instituto De Matemática e Estatística \\ DA \\ Universidade DE SÃo Paulo \\ PARA \\ OBTENÇÃO DO TÍTULO \\ DE \\ Mestre em CiÊnCIAS \\ Programa: Matemática Aplicada \\ Orientador: Prof ${ }^{\mathrm{a}}$. Dr ${ }^{\mathrm{a}}$. Joyce da Silva Bevilacqua
}

Durante o desenvolvimento deste trabalho o autor recebeu auxílio financeiro da CAPES.

São Paulo, Fevereiro de 2020 


\section{Mineração de Dados aplicada à tuberculose nos municípios do estado de São Paulo}

Esta versão da dissertação contém as correções e alterações sugeridas pela Comissão Julgadora durante a defesa da versão original do trabalho, realizada em 11/02/2020. Uma cópia da versão original está disponível no

Instituto de Matemática e Estatística da Universidade de São Paulo.

Comissão Julgadora:

- $\operatorname{Prof}^{\mathrm{a}}$. Dr ${ }^{\mathrm{a}}$. Joyce da Silva Bevilacqua (orientadora) - IME-USP

- Prof. Dr. Elias Roma Neto - FSEA-SENAC

- Prof. Dr. Francisco de Assis Zampirolli - UFABC 


\section{Agradecimentos}

Agradeço imensamente aos meus familiares, principalmente aos meus pais, Regina e Heraldo, e minhas irmãs Regiane e Cristiane por ter ficado ao meu lado durante todos os momentos de dificuldade e glória. Também agradeço à minha esposa Carliana por me mostrar que em momentos difíceis devemos ter fé e confiança.

Agradeço a minha orientadora Joyce, pelos seus ensinamentos, paciência, incentivo e por me aceitar como orientando desde a primeira vez que fui à sua sala, me acolhendo de braços abertos em todos os momentos.

Aos professores doutores do departamento que me motivaram e ensinaram durante e depois das disciplinas realizadas, principalmente aos professores: Antoine Laurain, Claudia Monteiro Peixoto, David Pires Dias, Jorge M. Sotomayor Tello e Sarajane M. Peres pelos conhecimentos transmitidos que foram válidos para a construção desse trabalho, meu desenvolvimento acadêmico e profissional.

Agradeço, também, aos meus amigos que estiveram ao meu lado durante todo esse período de estudo no instituto.

Agradeço também à empresa CAPES pelo apoio financeiro provido. 


\section{Resumo}

\section{SANTOS, Erick P. Mineração de Dados aplicada à tuberculose nos municípios do estado}

de São Paulo. 2020. 119 f. Dissertação (Mestrado) - Instituto de Matemática e Estatística, Universidade de São Paulo, São Paulo, 2020.

A tuberculose é uma doença que atinge cerca de 2 bilhões de pessoas em todo o mundo, com cerca de 10,4 milhões de novos casos a cada ano e 1,7 milhões de mortes. Apesar de ser uma doença que pode ser prevenida por vacina e curada através de tratamentos clínicos, sua taxa de incidência ainda é alta em alguns municípios do Brasil. Por ser uma doença de transmissão direta, é conhecida a influência de fatores socioeconômicos sobre a taxa de incidência. Motivados por esse panorama, o objetivo deste trabalho é propor uma metodologia para identificar e analisar relações entre dados socioeconômicos e de notificações da doença, utilizando técnicas de mineração de dados. Foram usados os bancos de dados da Fundação SEADE, IBGE e DATASUS relativos aos municípios do estado de São Paulo. Aos dados originais foram aplicados pré-processados com o uso de técnicas de imputação múltipla através do algoritmo Expectation Maximization com reamostragem; técnicas espaciais por meio do índice e o diagrama de Moran; discretização de valores contínuos e obtenção de padrões espaciais. Os municípios foram agrupados utilizando o algoritmo de agrupamento hierárquico exclusivo AGNES e os resultados validados com o uso do índice de Silhoutte e do coeficiente de correlação cophenético. Na etapa seguinte, o algoritmo APRIORI foi aplicado e extraídas regras de associações entre as variáveis. Os resultados obtidos por essa metodologia corroboram conhecimentos prévios sobre a doença e fatores socioeconômicos, tais como, IDHM, distância ao presídio, densidade de moradores por dormitório e despesas com saúde. No entanto, a análise espacial possibilitou a identificação de municípios que estão fora da lista de municípios de controle prioritário pelos órgãos de saúde, cujas taxas de incidência são crescentes assim como a de seus vizinhos. Esta pode ser uma indicação de um alerta para controles preventivos nessas localidades. A metodologia pode ser adaptada para inclusão ou substituição dos dados para tuberculose ou outra doença que tenha notificação no banco do DATASUS.

Palavras-chave: Mineração de Dados, Tuberculose, Regras de associação. 


\section{Abstract}

\section{SANTOS, Erick. P. Data Mining Applied to tuberculosis in São Paulo state municipali-}

ties. 2020. 119 f. Dissertation (Master's degree) - Institute of Mathematics and Statistics, University of Sao Paulo, São Paulo, 2020.

Tuberculosis is a disease that affects about 2 billion people worldwide, with about 10.4 million new cases each year and 1.7 million deaths. Despite being a disease that can be prevented by the vaccine and cured through clinical treatments, its incidence rate is still high in some municipalities in Brazil. As it is a disease of direct transmission, the influence of socioeconomic factors on the incidence rate is very well known. Motivated by this panorama, the objective of this work is to propose a methodology to identify and analyze relationships between socioeconomic data and disease reports, using data mining techniques. The SEADE, IBGE and DATASUS databases of the municipalities of the state of São Paulo were used. To the original data were applied pre-processing algorithms using multiple imputation techniques as the Expectation Maximization algorithm with boostrap; spatial techniques using the index and the Moran diagram; discretization of continuous values and identifications of spatial patterns. The municipalities were grouped using the exclusive hierarchical clustering algorithm AGNES and the results were validated using the Silhoutte index and the cophenetic correlation coefficient. In the next step, the APRIORI algorithm was applied and rules for associations between variables were extracted. The results obtained by this methodology corroborate previous knowledge about the disease and some socioeconomic factors, such as MHDI, distance to penitentiaries, density of residents per bedroom and health expenses. However, through spatial analysis, it has enabled the identification of municipalities that are outside the list of municipalities with priority control by health agencies, whose incidence rates are increasing as well as the incidences of their neighbors. This identification could anticipate preventive actions over these locations. The methodology can be adapted to include or replace data for tuberculosis or another disease that has been notified in the DATASUS database.

Keywords: Data mining, Tuberculosis, Association rules. 


\section{Sumário}

$\begin{array}{ll}\text { Lista de Abreviaturas } & \text { ix }\end{array}$

Lista de Símbolos $\quad$ xi

Lista de Figuras $\quad$ xiii

$\begin{array}{lc}\text { Lista de Tabelas } & \text { xvii }\end{array}$

1 Introdução $\quad 1$

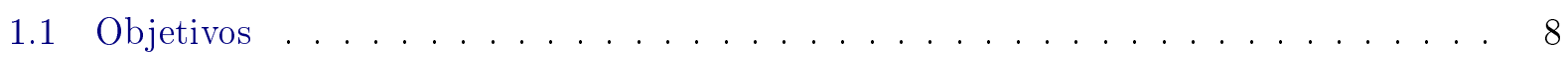

1.2 Organização do Trabalho . . . . . . . . . . . . . . . . . 8

2 Descoberta de Conhecimento em Base de Dados e Mineração de Dados 9

2.1 Os Dados em MD . . . . . . . . . . . . . . . . . . . . . . 10

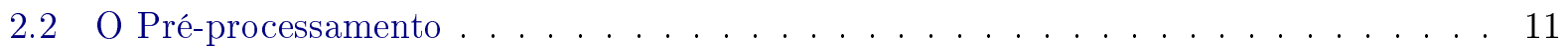

2.2.1 Tratamento dos Dados Faltantes . . . . . . . . . . . . . . . . 12

2.3 Sumarização . . . . . . . . . . . . . . . . . . . . . . . . 14

2.4 Análise de Predição (Classificação e Regressão) . . . . . . . . . . . . . . . . 15

2.5 Análise de Agrupamento . . . . . . . . . . . . . . . . . . . . . . . 15

2.5 .1 Validação dos Agrupamentos . . . . . . . . . . . . . . . 22

2.6 Associação . . . . . . . . . . . . . . . . . . . . . . 24

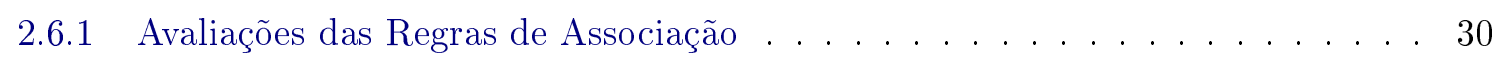

2.7 Análise de Dados Espaciais . . . . . . . . . . . . . . . . . . . . 32

3 Materiais e Metodologia $\quad 39$

3.1 Materiais . . . . . . . . . . . . . . . . . . . 39

3.1.1 Tratamento dos dados socioeconômicos . . . . . . . . . . . . . . . . 45

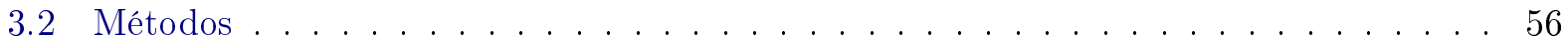

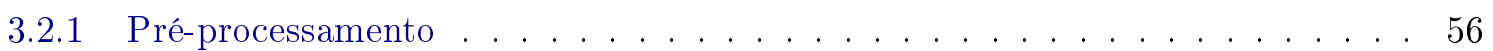

3.2.2 Análise Descritiva dos Dados . . . . . . . . . . . . . . . . 59

3.2.3 Agrupamento das Séries Temporais da incidência de TB . . . . . . . . . . . 62

3.2 .4 Aplicação da Análise de Associação . . . . . . . . . . . . . . . . . . . . 65

4 Resultados e Discussão $\quad 67$

4.1 Análise Descritiva dos Dados . . . . . . . . . . . . . . . . . . . 67

4.2 Análise de Agrupamento nos dados TS . . . . . . . . . . . . . . . . 70 
viii SUMÁRIO

4.3 Análise Espacial dos Dados . . . . . . . . . . . . . . . . . . . 73

4.4 Extração dos Padrões . . . . . . . . . . . . . . . . . . . . . 79

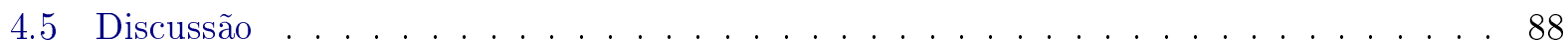

5 Conclusões $\quad 91$

5.1 Considerações Finais . . . . . . . . . . . . . . . . . . . . . . . . . . . 91

5.2 Sugestões para Pesquisas Futuras . . . . . . . . . . . . . . . . . . . . . 91

$\begin{array}{ll}\text { Referências Bibliográficas } & 93\end{array}$ 


\title{
Lista de Abreviaturas
}

\author{
AGNES AGglomerative NESting algoritmo \\ AIDS Acquired Immunodeficiency Syndrome \\ BCG Bacilo Calmette-Guérin \\ CNCT Campanha Nacional Contra a Tuberculose \\ COPPE Instituto Alberto Luiz Coimbra de Pós Graduação e Pesquisa em Engenharia \\ COREN Conselho Regional de Enfermagem \\ CPCC Coeficiente de Correlação cophenético \\ CRM Conselho Regional de Medicina \\ CRPHF Centro de Referência Professor Hélio Fraga \\ CSD Coeficiente de variação \\ DATASUS Departamento de Informática do SUS \\ EM Expactation Maximization algoritmo \\ FX Dados das Faixas Etárias \\ HIV Vírus da Imunodeficiência Adquirida (Human Immunodeficiency Virus) \\ IBGE Instituto Brasileiro de Geografia e Estatística \\ IC Intervalo de Confiança \\ IDHM Índice de Desenvolvimento Humano Municipal \\ IGM Índice Global de Moran \\ ILM Indice Local de Moran \\ KDD Descoberta de Conhecimento em Base de Dados (Knowledge Discovery in Database) \\ MD Mineração de Dados (Data Mining) \\ MDR-TB Multidrug resistant tuberculosis \\ MI Dados com as Médias das Incidências de TB nos últimos 3 anos \\ OMS Organização Mundial da Saúde \\ PNCT Plano Nacional de Controle da Tuberculose \\ SEADE Sistema Estadual de Análise de Dados \\ SINAN Sistema de Informação de Agravos de Notificação \\ SNT Serviço Nacional de Tuberculose \\ SUS Sistema Único de Saúde \\ SVS Secretaria de Vigilância em Saúde \\ TB Tuberculose (Tuberculosis) \\ TS Dados das Séries Temporais
}




\section{Lista de Símbolos}

$\begin{array}{ll}\mathbb{N} & \text { Conjunto dos números naturais } \\ \mathbb{R} & \text { Conjunto dos números reais } \\ \mathbb{R}_{+} & \text {Conjunto dos números reais positivos } \\ \mathbb{R}^{d} & \text { Conjunto de vetores d-dimensional sobre os reais } \\ \mathbb{R}^{N, d} & \text { Conjunto das matrizes N por d, cujo o corpo é } \mathbb{R} \\ \mathbf{x}, \mathbf{y}, \mathbf{z} & \text { Vetores de } \mathbb{R}^{d} \text { ou } \mathbb{R}^{N} \\ \mathbf{x}^{T} & \text { Vetor transposto } \\ N & \text { Número de objetos } \\ \mathcal{X} & \text { Conjunto de dados, ou } \mathcal{X}=\left\{\mathbf{x}_{i}\right\}_{i=1}^{N} \\ \mathcal{Y} & \text { Conjunto dos rótulos } \\ \mathbf{A} \in \mathbb{R}^{N, d} & \text { Matriz N por d com entrada real } \\ \mathbf{A} & \\ A_{i, j} \in \mathbb{R}^{d, N} & \text { Matriz inversa d por } \mathbf{N} \\ d i a g(\mathbf{A}) & \text { Elemento de uma matriz } \mathbf{A} \in \mathbb{R}^{N, d} . \text { Também escrito na forma } a_{i, j} \\ \sigma^{2} & \text { Vetor formado pela diagonal da matriz } \mathbf{A} \\ \sigma & \text { Variância. Também escrita como } V[\mathbf{x}] \\ \Sigma & \text { Desvio padrão. Também escrita como sd }[\mathbf{x}] \\ E[\mathbf{x}] & \text { Matriz de covariância } \\ E[\mathbf{x} \mid \mathbf{y}] & \text { Média amostral da variável } \mathbf{x} \in \mathbb{R}^{d} \text {. Também escrita como } \mu \text { ou } \overline{\mathbf{x}} \\ \mathcal{N}\left(\mu, \sigma^{2}\right) & \text { Média condicional de } \mathbf{x} \text { dado } \mathbf{y} \\ \mathcal{N}(\mu, \Sigma) & \text { Distribuição normal com média } \mu \text { e variância } \sigma^{2} . \\ \|\mathbf{x}\| & \text { Distribuição multivariada normal com média } \mu \text { e matriz de covariância } \Sigma \\ |A| & \text { Norma euclideana de um vetor } \mathbf{x} \in \mathbb{R}^{d} \\ \mathcal{O}(f) & \text { Número de elementos de um conjunto } A \text { discreto } \\ & \text { Ordem de complexidade } f \text { de um algoritmo de mineração }\end{array}$




\section{Lista de Figuras}

1.1 A Múmia de Nespaheran $([\operatorname{Mad} 04]$, p. 6) . . . . . . . . . . . . . . . . . 1

1.2 Retrato de Robert Koch $(1843$ - 1910) ([Wou10]) . . . . . . . . . . . . . . . . . . 2

1.3 A bactéria Mycobacterium tuberculosis, obtida pela microbiologista americana Janice Haney Car de um microscópio eletrônico ampliado 15549 vezes ([Car06]). . . . . 2

1.4 Compartimentos possíveis para uma pessoa contaminada pelo bacilo de Koch. . . . . 3

1.5 Internação de pacientes infectados por TB ([Wou10], p. 45) . . . . . . . . . . . . . 4

1.6 Linha do tempo dos principais fatos ocorridos sobre a tuberculose em conjunto com os controles no Brasil. . . . . . . . . . . . . . . . . . . . 6

1.7 Estimativa da taxa de incidência por 100 mil hab. feita pela OMS em 2017 ([Org18a],

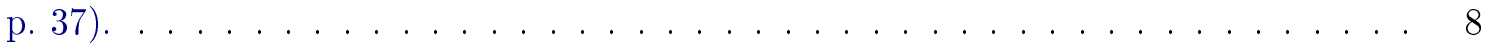

2.1 O processo do KDD adaptado de Fayyad et al. ([FPSS96], p. 41) . . . . . . . . . 9

2.2 Organização geral das tarefas de MD . . . . . . . . . . . . . . . . . . . . 11

2.3 Esquema da abordagem de imputação múltipla utilizando o algoritmo Expectation Maximization with Bootstrap (EMB) ([HKB11], p. 6) . . . . . . . . . . . . . . . 14

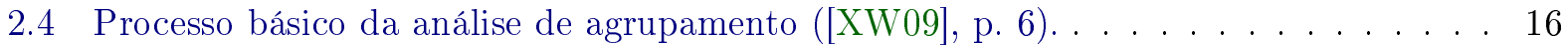

2.5 Tipos de abordagens dos algoritmos para análise de agrupamento. . . . . . . . . . . 20

2.6 Métricas de uniões de grupos utilizados na análise de agrupamento hierárquico. . . . 21

2.7 Ilustração de duas bases de dados utilizadas no algoritmo de busca por regras de associação. . . . . . . . . . . . . . . . . . . . . 25

2.8 Ilustração da complexidade na busca por regras de associação. . . . . . . . . . . . . 27

2.9 Poda dos itemsets infrequentes com base no Teorema 2.6.3 (Adaptado de Pang-Ning

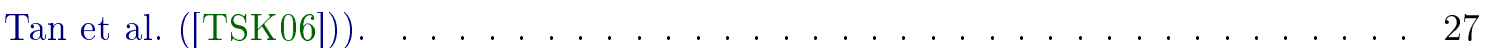

2.10 Ilustração da geração do itemset frequente utilizando o algoritmo APRIORI para um

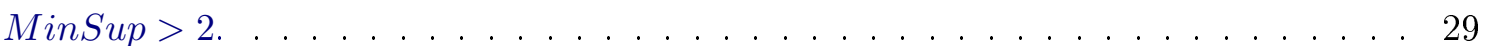

2.11 Conexões espaciais existentes nos municípios do estado de São Paulo. . . . . . . . . . 33

2.12 Distribuição dos municípios em relação ao número de links (ou vizinhos próximos) no estado de São Paulo. . . . . . . . . . . . . . . . . . . . . . 33

2.13 Gráfico da densidade gerada pelo índice de Moran obtidos pelas diferentes permutações ocorridas nos municípios, referente ao logaritmo da incidência de tuberculose

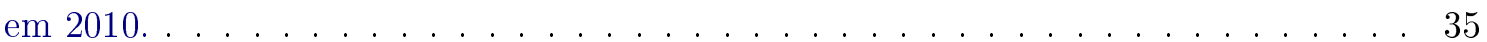

2.14 Ilustração dos quadrantes utilizados pelo Diagrama de Moran. . . . . . . . . . . . . 37

3.1 Fluxograma para a obtenção dos materiais que serão utilizados neste trabalho. . . . 40 
3.2 Metodologia para a redistribuição das taxas em cada faixa etária do IBGE e do DATASUS, com base nas categorias definidas pelo Estatuto da Criança e Adolescente e no Estatuto do Idoso.

3.3 Proporção de dados faltantes (lado esquerdo) e padrões dos dado faltantes em amarelo e não faltantes em azul (lado direito).

3.4 Parte 1: Distribuições dos dados observados (preto) e das médias dados faltantes para $\mathrm{m}=100$ (vermelho) das variáveis selecionadas.

3.5 Parte 2: Distribuições dos dados observados (preto) e das médias dados faltantes para $\mathrm{m}=100$ (vermelho) das variáveis selecionadas. . . . . . . . . . . . 51

3.6 Parte 1: Distribuição dos dados observados (preto) e dos dados completos (vermelho). 52

3.7 Parte 2: Distribuição dos dados observados (preto) e dos dados completos (vermelho). 53

3.8 Dendograma do agrupamento realizado através do algoritmo hierárquico AGNES para investigar as variáveis mais correlacionadas. . . . . . . . . . . . . . 54

3.9 Metodologia adotada para a extração dos padrões nos dados de tuberculose. . . . . . 56

3.10 Dendograma dos dados TS gerado pelo algoritmo AGNES com o parâmetro Average e métrica de dissimilaridade dada por $3.10 \ldots \ldots \ldots \ldots$. . . . . . . . 63

3.11 Variação do índice de Silhouette em relação ao número de grupos, ou cortes no dendograma gerado pelo algoritmo AGNES. . . . . . . . . . . . . . . 63

3.12 Resultado do índice de Silhouette para um agrupamento da série temporal na base TS, através do algoritmo hierárquico AGNES, com o parâmetro Average e a métrica definida pela equação $(3.10) \ldots \ldots \ldots \ldots \ldots \ldots$. . . . . . . . . . . . . . . .

3.13 Variação do número de regras e tempo de execução para os suportes: $3 \%, 5 \%, 10 \%$, $15 \%$ e $20 \%$.

4.1 Variação dos dados brutos sobre Casos Novos, Não se Sabe e Pós-óbito registrados no período de 2001 até 2017.

4.2 Box-plot da taxa de incidência dos municípios do estado de São Paulo com a taxa de incidência média no Brasil (linha tracejada em azul), taxa média de incidência no estado de São Paulo (linha sólida verde) e taxa média de incidência dos municípios (linha sólida vermelha), excluso o município de Borá e sem os outliers. . . . . . . . .

4.3 Os 20 municípios com as maiores taxas de incidência média registrada no período de 2001 até $2017 \ldots \ldots$. . . . . . . . . . . . . . . . . . . . . . . . . 69

4.4 Taxa de incidência anual do município de Borá, no período de 2001 até 2017 . . . . 70

4.5 Proporção da taxas de incidência em quatro municípios que apresentaram comportamentos diferentes ao longo do tempo. . . . . . . . . . . . . . . . . . . . 70

4.6 Box-plot das taxas de incidência normalizada e agrupadas segundo a metodologia proposta. A linha em vermelho é a média em cada ano. . . . . . . . . . . . . . . . 71

4.7 Agrupamento dos municípios de São Paulo gerado pelo algoritmo AGNES. . . . . . . 72

4.8 Gráfico de barras do índice global de Moran por ano no período analisado e seu valor descritivo obtido pelo método de Monte Carlo com 999 permutações dos municípios.

4.9 Mapa dos municípios de São Paulo com base nas classes do Diagrama de Moran obtidos pelo índice local que apresentaram correlações espaciais significativas (p-valor

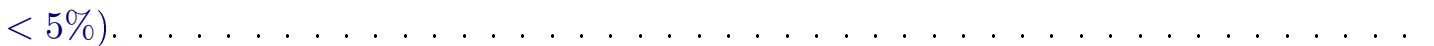


4.10 Diagrama de Moran da média das taxas de incidência no período de 2001 até 2017. A linha tracejada é a regressão linear feita pela média da taxa de incidência normalizada dos municípios e dos vizinhos. . . . . . . . . . . . . . . . . 75

4.11 Mapa dos municípios que estão localizados nos quadrantes do diagrama de Moran. 76

4.12 Parte 1: Mapa dos municípios de São Paulo após a utilização do método do diagrama de Moran. . . . . . . . . . . . . . . . . . . . 77

4.13 Parte 2: Mapa dos municípios de São Paulo após a utilização do método do diagrama de Moran. . . . . . . . . . . . . . . . . . . . 78

4.14 Categorias dos dados FX nos municípios de São Paulo. . . . . . . . . . . . . 78

4.15 Os municípios em destaque pertencem a regra R1. . . . . . . . . . . . . . . 79

4.16 Os municípios em destaque pertencem a regra $\mathrm{R} 2 \ldots \ldots \ldots$. . . . . . . 80

4.17 Os municípios em destaque pertencem a regra R3. . . . . . . . . . . . . 81

4.18 Os municípios em destaque pertencem a regra $\mathrm{R} 4 . \ldots \ldots \ldots$. . . . . . 81

4.19 Os municípios em destaque pertencem a regra R5. . . . . . . . . . . . . . 82

4.20 Os municípios em destaque pertencem a regra R6. . . . . . . . . . . . . 82

4.21 Os municípios em destaque pertencem a regra R7. . . . . . . . . . . . . 83

4.22 Os municípios em destaque pertencem a regra $\mathrm{R} 8 . \ldots \ldots \ldots$. . . . . . . 84

4.23 Os municípios em destaque pertencem a regra $\mathrm{R} 9 . \ldots \ldots \ldots \ldots$. . . . . . 85

4.24 Os municípios em destaque pertencem a regra R10 . . . . . . . . . . . . . 85

4.25 Os municípios em destaque pertencem a regra R11 . . . . . . . . . . . . 86 


\section{Lista de Tabelas}

2.1 Tabela com os intervalos para SC e suas interpretações (adaptado de [Ize08]). . . . . 23

3.1 Rótulos dos atributos da doença que foram utilizados para gerar a incidência de tuberculose, conforme regulamento no DATASUS. . . . . . . . . . . . . . . . 41

3.2 Tabela das bases de dados socioeconômicos. . . . . . . . . . . . . . . . . . . . 42

3.3 Rótulos e significados dos atributos socioeconômicas (Parte 1). . . . . . . . . . . . 44

3.4 Rótulos e significados dos atributos socioeconômicas (Parte 2). . . . . . . . . . . 45

3.5 Sumarizações de todas as variáveis socioeconômicas utilizadas neste trabalho, referente aos municípios do estado de São Paulo antes do tratamento dos dados faltantes. 46

3.6 Teste qui-quadrado para a identificação das variáveis com os dados faltantes relacionados formado através de uma tabela de contingência $2 \times 2$. Na diagonal superior, as letras $\mathrm{R}$ e $\mathrm{N}$ indicam relacionado e não relacionado, respectivamente. A diagonal inferior possui os p-valores do teste. . . . . . . . . . . . . . 48

3.7 Caracterização da base de dados Socio com os atributos socioeconômicos após o tratamento das variáveis. . . . . . . . . . . . . . . . . 55

3.8 Discretizações obtidas por diferentes métodos aplicados à variável da média da incidência nos últimos 3 anos $(2015,2016$ e 2017) . . . . . . . . . . . . . . . 57

3.9 Pré-processamento realizado em cada base de dados. . . . . . . . . . . . . . . 58

3.10 Sumarizações das variáveis temporal da incidência de tuberculose nos municípios do estado de São Paulo. Como não tem dados faltantes, a coluna Notif. indica o total de municípios que notificaram tuberculose no respectivo ano. . . . . . . . . . 59

3.11 Sumarizações de todas as variáveis socioeconômicas que serão analisadas neste trabalho, referente aos municípios do estado de São Paulo após o tratamento dos dados. 60

3.12 Variáveis com o número de municípios em cada categoria obtida pela metodologia do diagrama de Moran. . . . . . . . . . . . . . . . . . . 61

4.1 Número de municípios e as médias em cada grupo obtido pela execução do algoritmo de agrupamento hierárquico. . . . . . . . . . . . . . . . 71

4.2 Índice Global de Moran para as variáveis socioeconômicas. . . . . . . . . . . . . 76

4.3 Proporções de municípios segundo as faixas etárias obtidas dos dados FX. . . . . . . 77

4.440 municípios considerados de risco de tuberculose, após aplicar um limiar de 40 casos por 100 mil hab. para a variável MI e considerar o grupo 2 de tendência. A coluna Prioritários indica S para municípios que foram considerados como prioritários, conforme o PNCT e N caso contrário. Os locais de riscos foram assinalados com X. A coluna Regra indica em quais regras esses municípios se enquadram. . . . . . . . 87 


\section{Capítulo 1}

\section{Introdução}

A tuberculose é uma doença que acompanha a humanidade há muito tempo. O caso mais antigo registrado data de cerca de 1000 a.C é da múmia de Nespaheran, encontrada em 1910 por Sir Marc Armand Ruffer próximo à cidade de Tebas, localizada a $800 \mathrm{~km}$ do delta do Nilo ao sul de Alexandria. A Figura 1.1 mostra essa múmia, identificada como um sarcedote egípcio de Amun, de aproximadamente 30 anos e portador de TB espinhal, cujo o desenho foi feito por Mrs. Cecil M. Firth ([Mad04]).

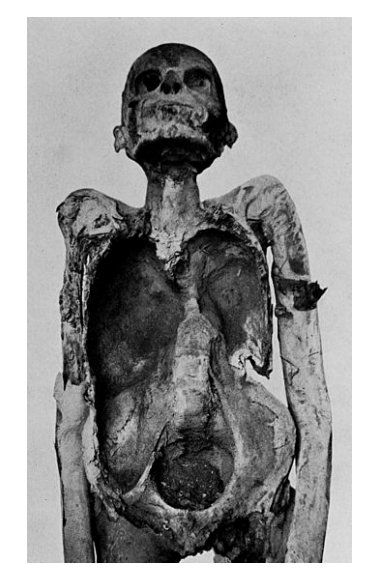

Figura 1.1: A Múmia de Nespaheran ([Mad04], p. 6).

A doença foi responsável por mais de $25 \%$ das mortes na cidade de Nova York, entre 1810 e 1815, devido aos fatores socioeconômicos, tais como, superlotação, má nutrição, falta de higiene, saneamento e falta de cuidados médicos. Após a revolução industrial as taxas de mortalidade começaram a cair, principalmente pela melhoria nas condições socioeconômicas, percepção de que a doença é infecciosa e início das internações em sanatórios ([Mur04]).

Jean Antoine Villemin (1827 - 1892), em 1865, estudou a inoculação do material retirado de tubérculos obtidos de seres humanos infectados. Em 1882, Robert Koch (1843 - 1910) mostrado na Figura 1.2, descobriu o agente causador da doença e por essa descoberta recebeu em 1905 o Prêmio Nobel de Medicina ([Wou10] e [Org18b]). Por esse motivo a bactéria é também conhecida como Bacilo de Koch. 


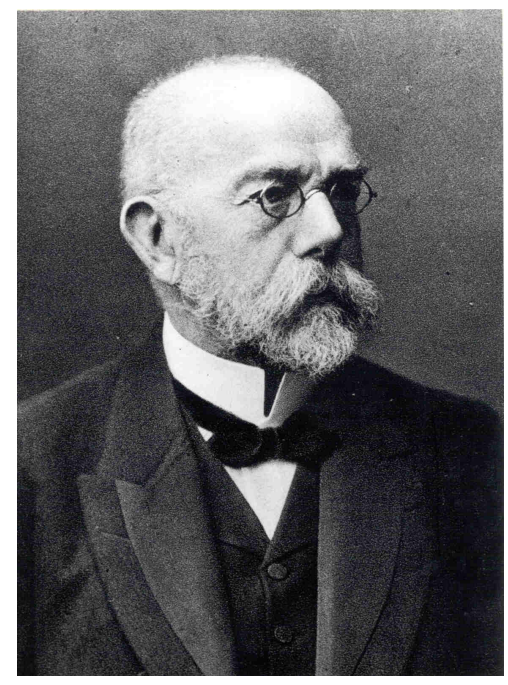

Figura 1.2: Retrato de Robert Koch (1843 - 1910) ([Wou10]).

A tuberculose é, portanto, uma doença bacteriana infectocontagiosa causada pela bactéria $M y$ cobacterium tuberculosis (Figura 1.3), propagando-se por transmissão direta entre humanos por via aérea em praticamente a totalidade dos casos ([Leo00] e [Mad04]). Afeta principalmente os pulmões, mas pode afetar outros órgãos ou sistemas, como a tuberculose cutânea, pleural, ganglionar, óssea, geniturinária, intestinal, meninges, ocular e hepática ([dPeTR1 ] e [Mac99]).

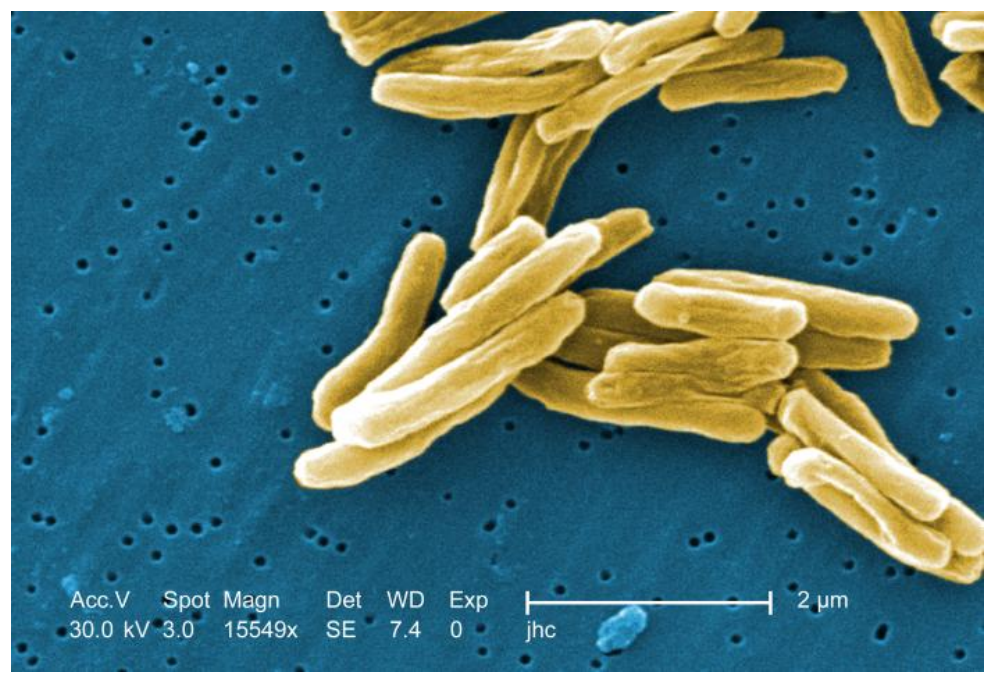

Figura 1.3: A bactéria Mycobacterium tuberculosis, obtida pela microbiologista americana Janice Haney Car de um microscópio eletrônico ampliado 15549 vezes ([Car06]).

Independente do órgão afetado, aproximadamente 1,7 bilhões de pessoas ( $23 \%$ da população mundial) possuem tuberculose na sua forma latente, ou seja, a bactéria está presente, mas o indivíduo não apresenta sintomas nem transmite a doença ([Org18a]). Entretanto, isto não significa que a doença permanecerá latente. Se ocorrer uma queda no sistema imunológico, os tubérculos latentes podem se tornar ativos e a doença se manifestar ([Wou10], [Wik18b] e [Org18b]).

Estima-se que ao tossir, uma pessoa com tuberculose ativa pode expelir até 3,5 milhões de bacilos num período de 24 horas. Cada partícula pode flutuar por um período de até 8 horas, depositandose sobre roupas, lenços, livros, móveis e na poeira. Ao instalar-se no organismo humano sadio, o 
bacilo permanece inativo por cerca de 3 dias e, após esse período, inicia-se o ciclo de reprodução que se renova a cada 18 horas. Simultaneamente, o corpo ativa o processo de defesa orgânica na tentativa de conter a infecção ([Fil01]).

Os sintomas da doença dependem do tipo de tuberculose adquirida. Para o caso mais comum, de TB pulmonar, os sintomas são dores no peito, ao respirar, tosse persistente por 3 semanas com ou sem sangue, fadiga, febre vespertina que costuma não ultrapassar $38,5{ }^{\circ} \mathrm{C}$, sudorese noturna e emagrecimento. Na TB extrapulmonar, os sintomas variam, sendo comum a todas elas febre vespertina, sudorese noturna, perda de apetite e cansaço ([Boa16]). A Figura 1.4 ilustra como a doença se apresenta na população infectada.

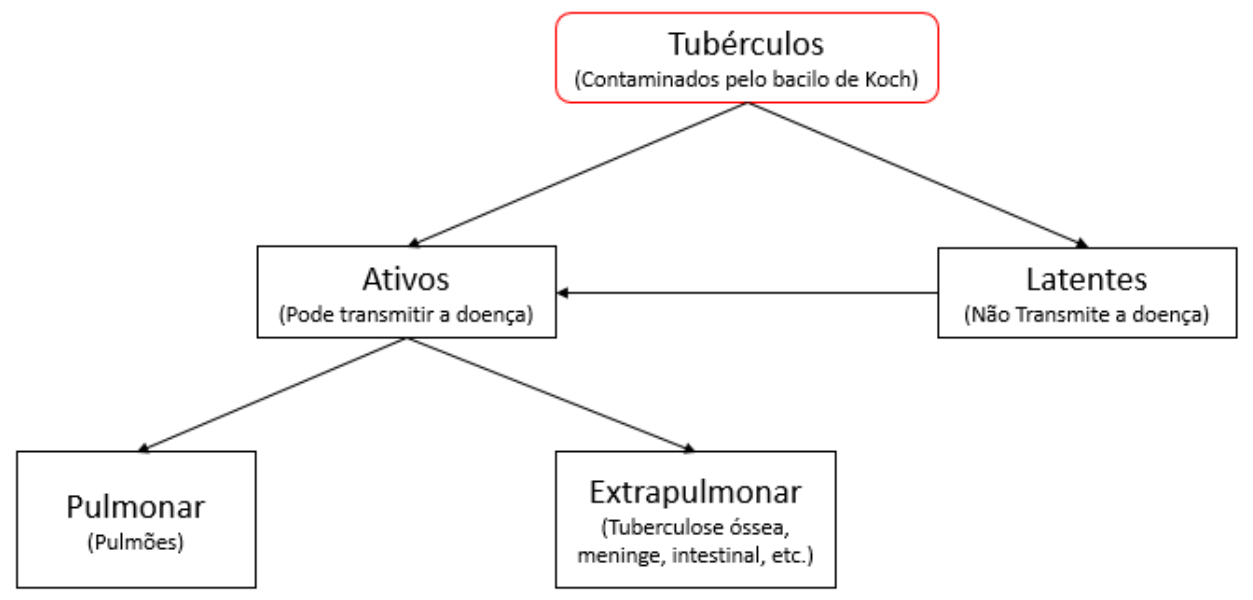

Figura 1.4: Compartimentos possiveis para uma pessoa contaminada pelo bacilo de Koch.

O diagnóstico pode ser feito por meio da baciloscopia do escarro, o radiológico e a prova tuberculina ([SdSdEdP1 ], [dSdEdSaP1 ] e [dSJ04]).

Antes da descoberta dos antibióticos por volta de 1940, o isolamento dos doentes em sanatório e hospitais promoveu significativa diminuição do número de infectados ([Dan06] e [Wou10]). A Figura 1.5 ilustra como as pessoas infectadas ficavam acomodadas. Nesta época, o tratamento consistia em repouso e boa alimentação e, em alguns casos, tentativas de tratamentos cirúrgicos ([CdSK02]).

A partir da descoberta dos antibióticos isoniazidas (1912 por H. Meyer e J. Mally [Smi05]) e estreptomicina (1944 por Selman Abraham Waksman et al. [SBW44]), o tratamento combinado dessas drogas proporcionou a cura da doença a partir de 1952. Atualmente é também utilizada a rifampicina reduzindo o tratamento de 12 para 6 meses ([Mad04] e [MSH15]).

Em 1921, no Instituto Pasteur em Lille na França, Jean-Marie Camille Guérin (1872 - 1961) e Léon Charles Albert Calmette (1863 - 1933) isolaram um bacilo bovino atenuado, permitindo o desenvolvimento da vacina BCG - Bacillus Calmette-Guérin que não impede a infecção, mas evita as formas mais graves da doença, como a tuberculose miliar e meningite ([Mad04] e [Sau18]). 


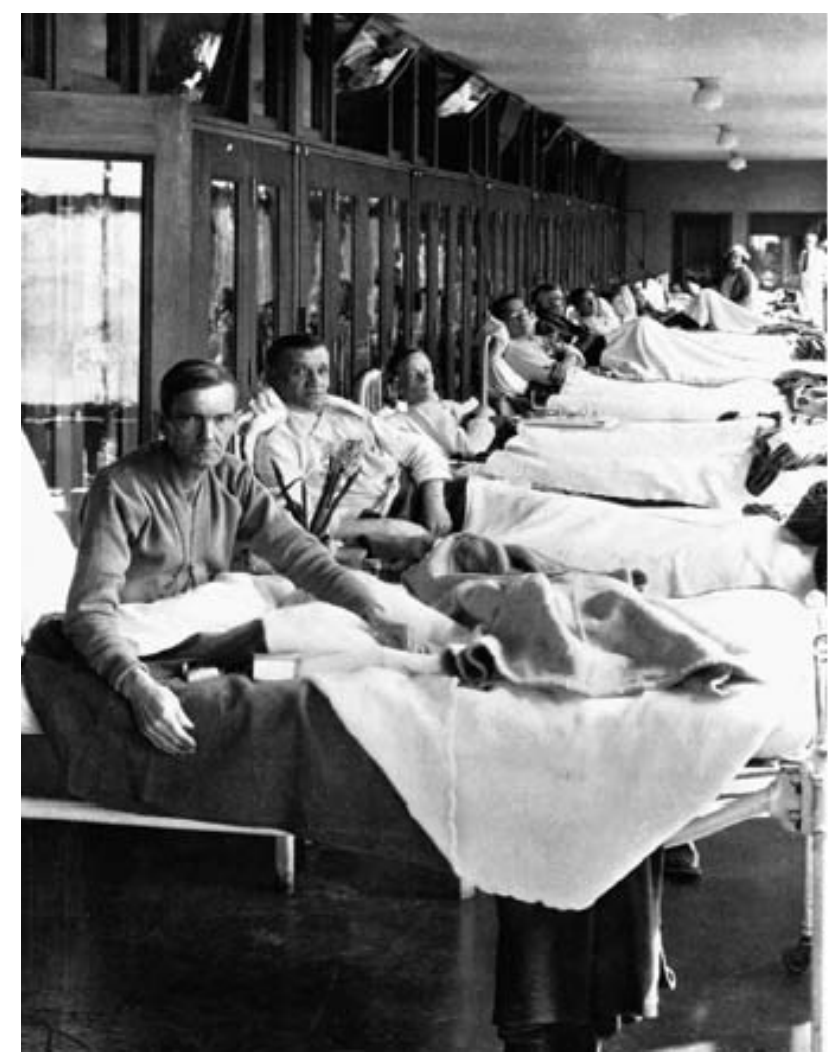

Figura 1.5: Internação de pacientes infectados por TB ([Wou10], p. 45).

\section{A Tuberculose no Brasil}

A tuberculose está presente desde o Brasil colonial. Muitos padres e missionários eram portadores ou morreram da doença, dentre eles os padres Manoel da Nóbrega e José de Anchieta ([CdSK02]).

A luta contra a TB começa a ser articulada em 1899 pela Liga Brasileira Contra a Tuberculose e pela Liga Paulista Contra a Tuberculose, por meio do estímulo à criação de sanatórios e hospitais, iniciando a parceria com as instituições privadas ([HGTP07]). A Liga Paulista Contra a Tuberculose inaugurou em 1932, nas proximidades da capital paulistana (Jaçanã), o primeiro hospital sanatório com capacidade inicial de 100 leitos ([GC39] e [dMdSaP17]).

Em 1936, Manoel D. de Abreu (1891 - 1962), médico especializado em radiologia, em pesquisas realizadas no Hospital Laennec em Paris, desenvolveu um novo método de diagnóstico radiográfico miniaturizado do tórax, popularmente conhecido como abreugrafia ([dS63] e [HGTP07]).

Em 1940, é lançado o Serviço Nacional de Tuberculose (SNT) e em 1946 a Campanha Nacional Contra a Tuberculose (CNCT) com o objetivo de atuar em 66 municípios onde ocorriam 80\% dos óbitos e isolar 22 mil focos, criar 14186 leitos hospitalares, criar um modelo de sanatório popular, instalar um dispensário por 100 a 120 mil habitantes, promover a preparação de pessoal, incrementar a vacinação BCG e promover o desenvolvimento científico e tecnológico ([HGTP07]).

Em 1993, a OMS declarou a tuberculose uma emergência mundial e em resposta o Brasil criou em 1994 o Plano Emergencial para Controle da Tuberculose, elegendo 230 municípios prioritários, 
segundo critérios epidemiológicos (incidência, mortalidade e associação com HIV). A meta era diagnosticar, no mínimo, 90\% dos casos e curar, pelo menos, $85 \%$ dos casos diagnosticados ([HGTP07]).

Atualmente o tratamento é realizado diretamente e exclusivamente pelo Sistema Único de Saúde (SUS), no qual o paciente é acompanhado durante todo o tratamento (Tratamento Diretamente Observado), que dura de 6 meses a um ano ([SdSdEdP1 ] e [dSdEdSaP1 ]).

Em 1998, o Centro de Referência Prof. Hélio Fraga (CRPHF) em conjunto com o Núcleo de Centros de Excelência da COPPE da Universidade Federal do Rio de Janeiro, elaboraram um planejamento estratégico para o controle da TB no Brasil. No ano seguinte, o Ministério da Saúde lançou o Plano Nacional de Controle da Tuberculose (PNCT) que deu continuidade ao plano de 1994, ampliando para todo o território nacional. O PNCT permitiu um monitoramento mais fidedigno das dificuldades operacionais das ações de controle municipais, contribuindo para definição de novas estratégias de combate à TB. Dados de coeficientes de incidência de HIV, municípios com altas densidades populacionais, limiares superiores a média nacional, taxa de resistência adquirida ou multirresistência, entre outros, indicam os municípios que merecem atenção especial ([dVeS01]).

Em 2000, o Ministério da Saúde lançou o Plano Nacional de Mobilização para eliminação da Hanseníase e Controle da Tuberculose em Municípios Prioritários por meio da Atenção Básica e em 2001, deu-se início ao Plano Estratégico para Implementação do Plano de Controle da Tuberculose no Brasil ([HGTP07], p. 56).

Em 2003 houve a criação da Secretaria de Vigilância em Saúde (SVS) que passou a coordenar várias atividades e estabelecer um plano estratégico comum à saúde pública trazendo vantagens para a integração de áreas técnicas, esferas dos governos municipais, estaduais e federais, envolvidas na vigilância e controle de agravos à saúde ([HGTP07]).

Em 2014, foi implantado um sistema TBWEB que permite o acompanhamento online do paciente desde sua entrada até a finalização do tratamento e contorna, quase que completamente, as questões de duplicidade ([Gua11]). Em 2017, foi aprovado o Plano Nacional pelo Fim da Tuberculose como Problema de Saúde Pública ([dVeS06]), cujas metas até 2035 são:

- Reduzir os coeficientes de incidência da doença para menos de 10 casos a cada 100 mil habitantes;

- Reduzir a mortalidade para menos de um óbito a cada 100 mil habitantes.

O objetivo é cumprir essas metas em paralelo com os planos indicados pela OMS. Essa iniciativa começou em 2018 com a Implementação do Plano Nacional pelo Fim da Tuberculose como Problema de Saúde Pública no Brasil: primeiros passos rumo ao alcance das metas ([dVeS18]).

A Figura 1.6 mostra uma síntese da linha do tempo com as tentativas de controle da tuberculose no Brasil, demonstrando a relevância do estudo da doença e de indicadores de focos de forma precoce e eficiente, o que inspirou a abordagem deste trabalho. 


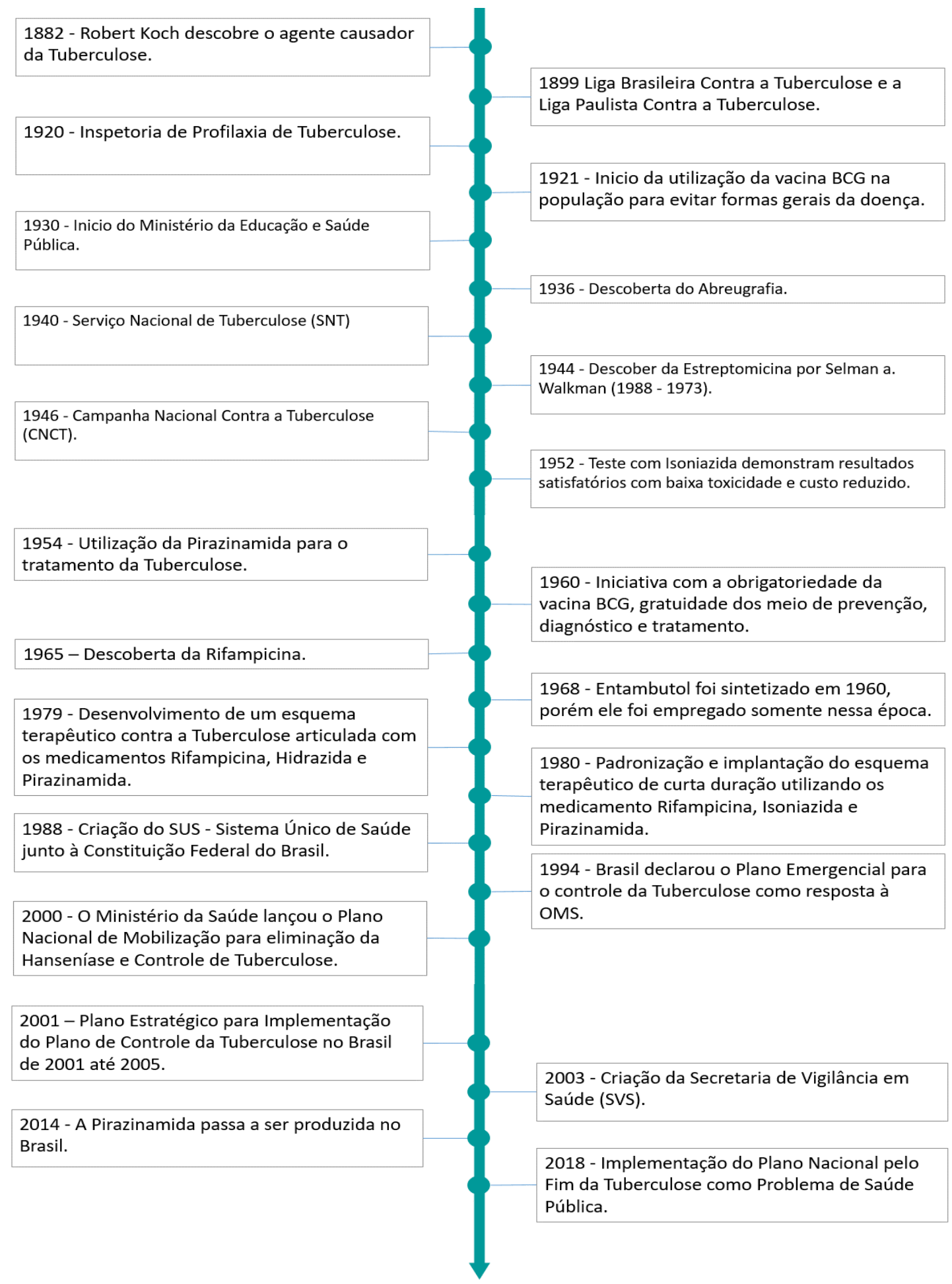

Figura 1.6: Linha do tempo dos principais fatos ocorridos sobre a tuberculose em conjunto com os controles no Brasil. 
Para concluir, a tuberculose tem um histórico de morte muito agressivo e muitas pessoas do meio acadêmico tiveram suas vidas precocemente interrompidas por causa da doença.

- Srivinasa Ramanujan (1887 - 1920) um matemático indiano que contribuiu imensamente para a teoria dos números e motivou muitos matemáticos contemporâneos a investigarem fórmulas que descobrira sozinho e outras com a ajuda de seu professor e amigo matemático Hardy.

- Niels Henrik Abel (1802 - 1829) um matemático norueguês que morreu jovem aos 26 anos devido à desnutrição e tuberculose. Seu trabalho proporcionou o surgimento de várias linhas de pesquisa.

- Ferdinand Gotthold Max Eisenstein (1823 - 1852), matemático alemão, especialista em teoria dos número e análise, morreu de tuberculose com 29 anos de idade, pois foi contaminado na prisão da Prússia. Sua detenção ocorreu devido suas atividades revolucionárias em Berlim.

- Erwin Rudolf Josef Alexander Schrödinger (1887 - 1961) físico austríaco, conhecido principalmente pelas suas contribuições em mecânica quântica, recebeu o prêmio Nobel de Física em 1933. Morto em Viena aos 73 anos por tuberculose.

- Lev Semyonovich Vygotsky (1896 - 1934) psicólogo soviético responsável por diversas teorias sobre educação e aprendizado, faleceu de tuberculose aos 37 anos.

- Dom Pedro ou Dom Pedro I (1798 - 1834) foi o imperador do Brasil de 1822 até 1831. Morreu de tuberculose aos 36 anos alguns meses depois dele e os liberais terem se saído vitoriosos após uma guerra contra seu irmão Miguel.

Acreditava-se que a tuberculose estaria erradicada, ou pelo menos, confinada aos países pobres até o final do século XX ([CdSK02]). Entretanto, tal fato está longe de acontecer. Movimentos migratórios populacionais, deficiência no sistema público de saúde, uso inadequado de medicamentos, crises sociais e financeiras, doenças como AIDS, HIV, diabetes, alcoolismo, tabagismo, medicamentos imunossupressores e drogas ilícitas colaboram para o surgimento de novos casos de tuberculose.

Segundo o relatório da OMS - Organização Mundial da Saúde ([Org18a]) em 2017, 10 milhões de pessoas contraíram tuberculose em todo o mundo e, aproximadamente 1,6 milhões vieram a óbito, sendo que 300 mil também eram portadoras de HIV. Cerca de 558 mil desenvolveram resistência a pelo menos um dos antibióticos.

A Figura 1.7 mostra a distribuição das taxas de incidência de tuberculose no mundo. Oito países respondem por $66 \%$ do total de casos, com taxas superiores a 200 casos novos por 100 mil habitantes: Índia (27\%), China (9\%), Indonésia (8\%), Filipinas (6\%), Paquistão (5\%), Nigéria (4\%), Bangladesh (4\%) e África do Sul (3\%). No Brasil, a taxa de incidência é de 133 casos por 100 mil habitantes e é considerado um dos 20 países prioritários para coinfecções TB-HIV. 


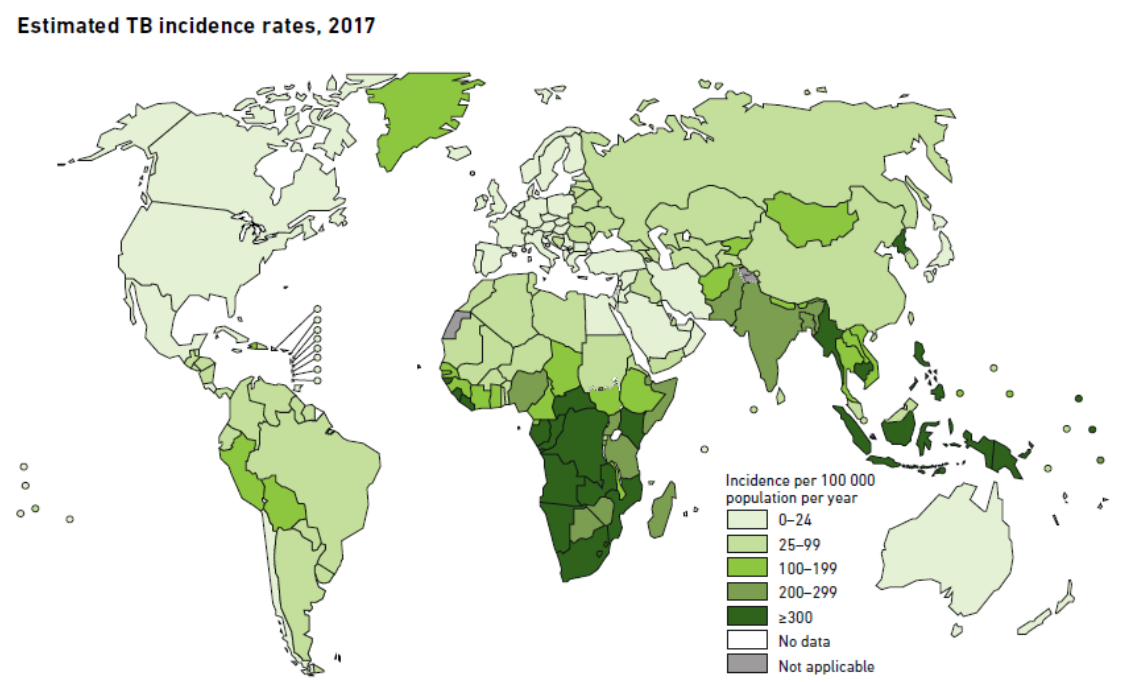

Figura 1.7: Estimativa da taxa de incidência por 100 mil hab. feita pela OMS em 2017 ([Org18a], p. 37).

\section{$1.1 \quad$ Objetivos}

O objetivo principal deste trabalho é propor uma metodologia baseada em técnicas de mineração de dados, que possibilite identificar fatores socioeconômicos que influenciam as taxas de incidência de tuberculose municipais.

Para essa análise, objetivos secundários deverão ser atingidos:

- Consolidação de uma base ampla de dados, agregando dados socioeconômicos aos dados municipais de incidência de tuberculose;

- Implementação de técnicas de ajustes de parâmetros para viabilizar a integração das informações da base de dados;

- Implementação de regras de associação;

- Análise de padrões e indicação de risco.

O ambiente computacional utilizado é o R-Studio para processar, organizar e analisar os dados.

\subsection{Organização do Trabalho}

O texto está organizado da seguinte forma: no capítulo 1, uma revisão sobre mineração de dados e a apresentação dos algoritmos de utilizados neste trabalho. O capítulo 2, apresenta os filtros aplicados às bases de dados e a metodologia proposta. No capítulo 3 , resultados e discussões. Finalizando, as conclusões e referências bibliográficas. 


\section{Capítulo 2}

\section{Descoberta de Conhecimento em Base de Dados e Mineração de Dados}

A Descoberta de Conhecimento em Base de Dados ou Knowledge Discovery in Database (KDD) foi abordado inicialmente por Gregory Piatetsky-Shapiro em 1989. Seu surgimento foi para tentar tratar e analisar uma quantidade massiva de dados com o objetivo de buscar padrões e conhecimentos contidos nele. Usama Fayyad et al. (1996), define KDD como um processo não trivial para a identificação de padrões válidos, novos, compreensíveis e potencialmente úteis em dados ([FPSS96]).

O KDD envolve várias etapas e para cada etapa existem decisões e métodos que são aplicados para extrair diferentes padrões no conjunto de dados. Esse processo é interativo, pois a ocorrência de uma etapa depende da anterior (Figura 2.1). Ele inicia por entender o objetivo, do ponto de vista do cliente, e estudar os dados que serão utilizados. Aos dados selecionados são aplicados métodos e técnicas de pré-processamento e transformação, para sua consolidação e preparação para a etapa da busca por padrões. Em seguida é realizado o pós-processamento, o qual avalia e analisa os padrões obtidos, gerando conhecimento sobre os dados. Atualmente, o cientista de dados é o profissional que se aproxima mais dessa perspectiva de trabalho.

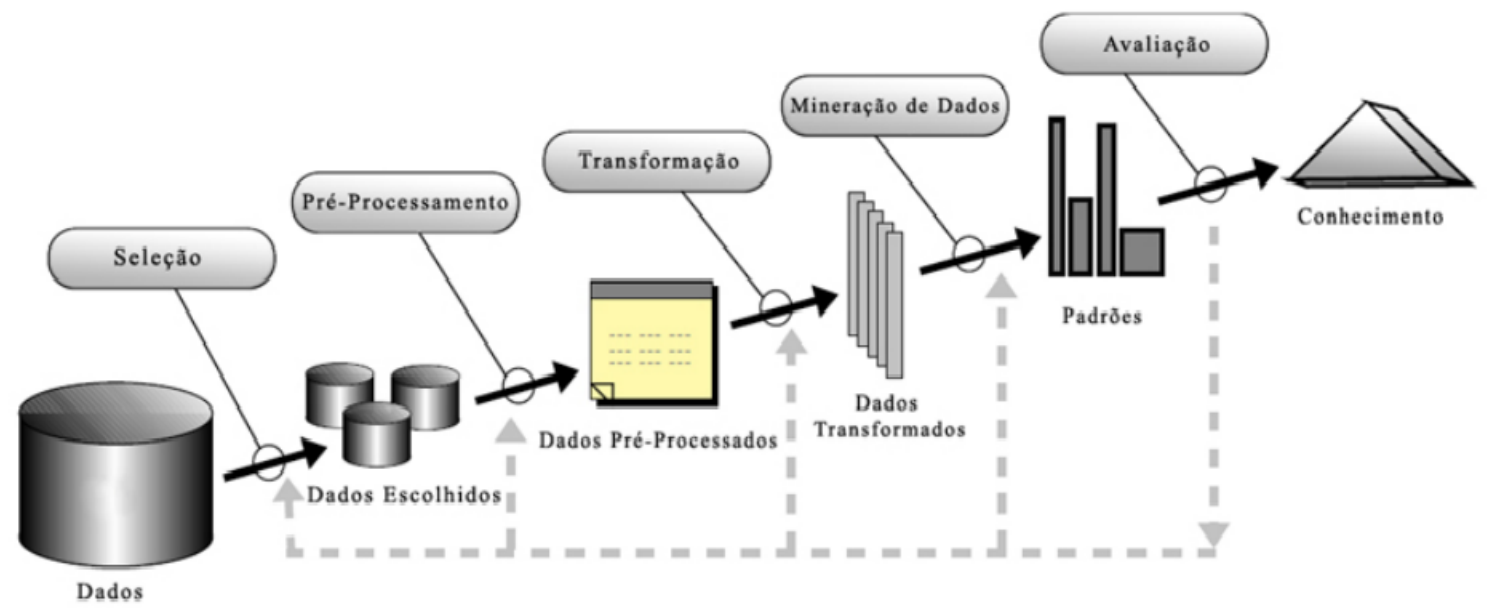

Figura 2.1: O processo do KDD adaptado de Fayyad et al. ([FPSS96], p. 41). 
A Mineração de Dados ou Data Mining é uma etapa do processo do KDD, na qual métodos inteligentes são aplicados para extrair padrões dos dados. Entende-se por métodos inteligentes formas automáticas ou semiautomáticas de encontrar padrões em dados, os quais devem ser submetidos a análises de especialistas para tomadas de decisões ([CdS09]).

Como a MD é a etapa que se destaca na descoberta de conhecimento, ela é muitas vezes confundida como sinônimo de KDD, conforme discutido por Jiawei Han e Micheline Kamber ([HK06]). A prática de examinar os dados já coletados e tratados a fim de extrair um padrão consistente é comum em outras áreas do conhecimento como Estatística, Machine Learning, Reconhecimento de Padrões, etc.

Os métodos automáticos são divididos em tarefas e a escolha depende do que procuramos realmente nos dados. Ora queremos descrevê-los ou entendê-los, ora inferi-los. Cada tarefa possui um conjunto de algoritmos que permite extrair padrões consistentes. A escolha do algoritmo está relacionada com a capacidade de manipular certos tipos de dados, o formato dos dados de entrada do algoritmo, compreensibilidade do modelo de saída, nível de familiaridade do usuário, escalabilidade, requisitos de software e hardware ([CVZH97]).

As tarefas de MD podem ser classificadas em duas categorias: Descritivas e Preditivas (Figura 2.2). A primeira procura entender ou descrever os dados, descobrindo propriedades e relações entre variáveis ou certo subconjunto de dados. Já a parte preditiva, permite inferir valores futuros ou desconhecidos de outras variáveis ([HK06]).

As tarefas preditivas podem ser divididas em duas categorias, sendo: Classificação, como uma predição categórica e Regressão como uma numérica. Enquanto que as tarefas descritivas são: Agrupamento, Sumarização, Modelagem de dependências (regras de associação) e Detecção de Desvios ([TSK06]).

Abaixo serão descritos os dados e algumas das tarefas utilizadas em MD, detalhando, principalmente, as tarefas descritivas e os algoritmos que serão utilizados neste trabalho.

\subsection{Os Dados em MD}

Os dados utilizados em MD podem ser Estruturados e Não-Estruturados. O primeiro tratase de uma organização tabular, com linhas e colunas, tais que as linhas são chamadas de objetos, instâncias, observações ou elementos e as colunas são os atributos, características ou variáveis ([TSK06] e [dSPB16]). Neste trabalho, as observações serão tratadas ora como séries temporais, ora como municípios do estado de São Paulo. Os dados Não-Estruturados, apresentam-se na forma não tabular, por exemplo, dados textuais, vídeo, imagem, áudio, entre outros ([Zik18]). Além dessa classificação, as variáveis dos dados estruturados podem ser qualitativa nominal ou ordinal, ou quantitativa discreta ou contínua.

Em termos matemáticos, um Conjunto de Dados ou uma Base de Dados, na forma estru- 


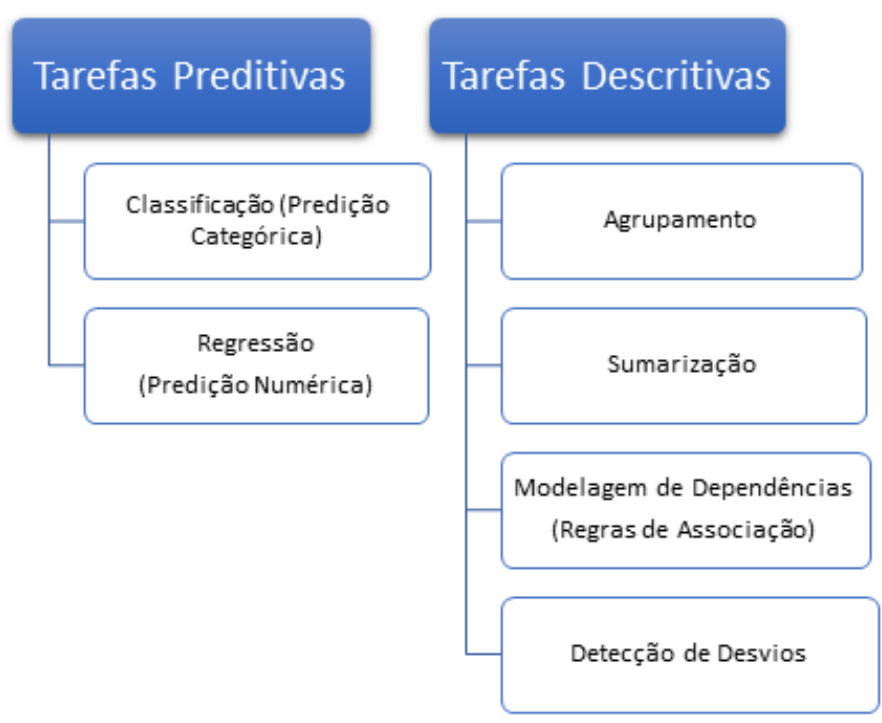

Figura 2.2: Organização geral das tarefas de $M D$.

turada, é definido como $\mathcal{X}=\left\{\mathbf{x}_{1}, \mathbf{x}_{2}, \ldots, \mathbf{x}_{N}\right\} \subset \mathbb{R}^{d}$, sendo $N \in \mathbb{N}$ é a quantidade de objetos e $d \in \mathbb{N}$ são os atributos.

Os atributos podem ser quantitativos, quando são valores numéricos ou qualitativos, não possuem valores numéricos. Há também subdivisões dessas classes, tais como, as variáveis quantitativas discretas que, de modo geral, podem ser vistas como resultantes de contagens, assumindo valores inteiros e as variáveis quantitativas contínuas que assumem valores em intervalos de números reais. As variáveis qualitativas ordinais, que possuem uma ordenação natural, indicam intensidade crescente de realização, as variáveis qualitativas nominais, são aquelas que não estabelecem uma ordem natural. Há também as variáveis qualitativas binárias que assumem apenas dois valores, em geral 0 ou 1.

\subsection{O Pré-processamento}

O pré-processamento é parte do processo de KDD e tem por objetivo melhorar a qualidade dos dados como um todo, favorecendo as análises dos resultados e também as aplicações dos algoritmos de mineração. O processo de KDD funciona conforme a regra 80-20, onde $80 \%$ está alocado na preparação dos dados e $20 \%$ nas análises ([McC07]). A preparação é constituída por:

- Limpeza dos dados: corrige inconsistência nos dados, tais como, valores faltantes, outliers e suavização de dados ruidosos;

- Integração: inclui dados de várias fontes na base de dados de estudo;

- Transformação: transforma os dados para que fiquem na mesma escala através de normalização e categorização dos dados numéricos;

- Redução: aplica-se técnicas de seleção e redução de características, amostragem e outras técnicas afim de reduzir a dimensionalidade dos dados. 
Existem inúmeras técnicas de pré-processamento capazes de melhorar a qualidade dos dados. Neste trabalho, diferentes técnicas foram aplicadas para tratar os dados de TB. Algumas dessas técnicas serão descritas no próximo capítulo durante a manipulação dos dados.

A redução das variáveis foram feitas através da análise de multicolinearidade. Ela permite identificar variáveis que estão correlacionadas, reduzindo a dimensionalidade do problema e o espaço de busca dos algoritmos. Neste trabalho, foi aplicado essa metodologia para detectar variáveis relacionadas com o uso do algoritmo de agrupamento. A métrica e os parâmetros do algoritmo foi guiado pelas medidas de avaliação, conforme será descrito posteriormente ([JBBA09]).

\subsubsection{Tratamento dos Dados Faltantes}

Em relação aos dados faltantes, eles são comuns em base de dados reais, podendo ocorrer devido a falta de registros, perda dos dados ou a má organização na coleta ou manipulação dos mesmos ([HK06]). Conforme Donald B. Rubin ([Rub76]), existem três mecanismos diferentes que podem gerar os dados faltantes, eles são definidos como:

- MCAR - Missing Completely at Random: os dados faltantes surgem de modo completamente aleatório e, neste caso, o conjunto de dados observados não tem nenhuma influência sobre os dados faltantes;

- MAR - Missing at Random: os dados observados tem influência nos dados faltantes;

- NMAR - Not Missing at Random: os dados faltantes não são aleatórios e eles dependem de eventos que não são observados ou controlados ([Ass12]).

Diferentes metodologias são aplicadas para cada mecanismo e testes estatísticos podem ajudar a identificar se os dados são MAR ([Ass12]). Já técnicas mais sofisticadas auxiliam na identificação se os dados são NMAR. O teste mais comum para identificar se os dados são MAR é o Qui-quadrado. Nela, utilizamos uma tabela de contingência para cada par de variáveis indicando o número de dados faltantes e aplica-se a equação (2.1), de modo que, $o_{i, j}$ e $e_{i, j}$ são os dados observados e esperados, respectivamente.

$$
q_{c}=\sum_{i=1}^{2} \sum_{j=1}^{2} \frac{\left(\left|o_{i, j}-e_{i, j}\right|-0.5\right)^{2}}{e_{i, j}} .
$$

A equação (2.1) calcula o teste qui-quadrado com correção de Yates, aplicado quando a amostra é pequena, usualmente menor do que 40, ou quando a frequência esperada de uma classe for menor que 5. Caso $q_{c} \in R C$, então há uma relação de dependência significativa, demonstrando que os dados são MAR. A região crítica, definida pela equação (2.2), é a região formada pela distribuição qui-quadrada com 1 grau de liberdade, visto que a tabela de contingência é sempre $2 \times 2$.

$$
R C=\{w: w \geq 3,841\} .
$$

Esse teste verifica se os dados faltantes em uma variável estão relacionados com os dados faltantes de outras variáveis, identificando relações de dependência, sendo possível também verificar 
a qualidade dos dados.

As estratégias comumente utilizadas para tratar os dados faltantes ([HK06], p. 61-62) são:

(i) Ignorar o dado: elimina-se o objeto ou atributo com os valores faltantes. Esse processo não é eficiente caso o número de objetos seja pequeno e, além disso, eliminar um objeto pode retirar informações valiosas dos dados durante o processo de mineração;

(ii) Preencher o valor ausente manualmente: inclui um valor específico no objeto que possui dados faltantes, utilizado apenas em tabelas pequenas. A técnica pode introduzir um grande viés por ser altamente dependente da pessoa que completa a tabela;

(iii) Uso de uma constante global no dado faltante: substitui todos os dados faltantes por um caractere, por exemplo, "NA";

(iv) Uso da média do atributo no dado faltante: substitui todos os dados faltantes pela média dos dados observados da mesma variável;

(v) Uso da média do atributo para todas as amostras pertencentes à mesma classe que o objeto especificado: substitui os dados faltantes pela média dos vizinhos mais próximos daquele objeto;

(vi) Uso do valor mais provável: caso os dados sejam categóricos o valor da moda pode ser aplicado em substituição dos dados faltantes. A utilização de técnicas como regressão, inferência Bayesiana e árvores de decisão, podem ajudar a estimar esse valor.

Há outras técnicas de tratamento de dados faltantes utilizados em MD, como mostram os trabalhos de Donald B. Rubin ([Rub87]), Heijden et al. ([HGDASTMK06]) e Luciana Nunes ([Nun07]) que estudam as diversidades no tratamento de dados faltantes com o foco em imputação múltipla.

A metodologia adotada neste trabalho para tratar os dados faltantes consiste na utilização do algoritmo EM - Expectation Maximization, conforme Dempster et al. ([DLR77]), com o uso de reamostragem com substituição nos dados observados (técnica Bootstrap), a fim de estimar diferentes valores faltantes numa mesma observação (Figura 2.3). Em seguida, utiliza-se o estimador da média incluindo os valores faltantes estimados.

O algoritmo EM tem como suposição que os dados são MAR - Missing at Random, ou seja, os dados faltantes dependem apenas dos dados observados e que os dados são oriundos da distribuição normal multivariada. O algoritmo procura completar os dados faltantes utilizando as informações dos dados observados através de dois passos, sendo o primeiro Expectation (E) que calcula o valor esperado da razão da verossimilhança para os dados completos e depois o passo Maximization (M), que procura encontrar o parâmetro ótimo que maximiza essa função.

Segundo Trevor Hastie et al. (2001), no passo E os dados faltantes podem ser estimados pela equação (2.3), na qual, os valores $i, o_{i}, m_{i}, \hat{\mu}$ e $\hat{\Sigma}$ são, respectivamente, a instância, a variável dos dados observados, a variável dos dados faltantes, a média e a matriz de covariância amostral. O 


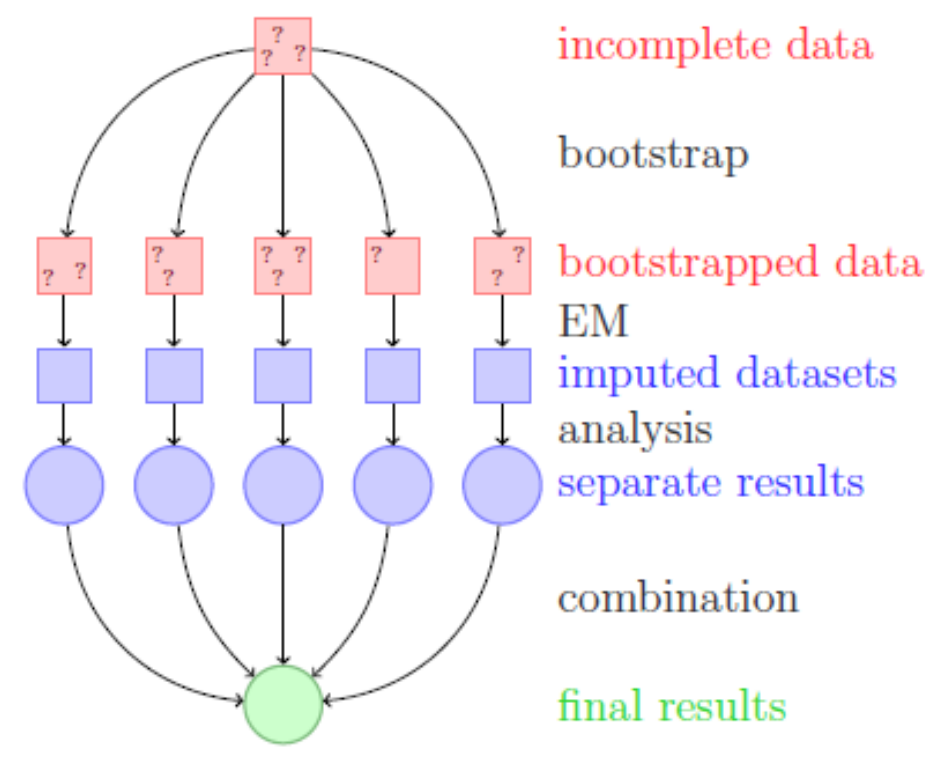

Figura 2.3: Esquema da abordagem de imputação múltipla utilizando o algoritmo Expectation Maximization with Bootstrap (EMB) ([HKB11], p. 6).

passo M atualiza a média e a covariância com os dados já imputados. O parâmetro $\theta$ define os parâmetros da distribuição considerada que, neste caso, é a distribuição normal.

$$
\hat{x}_{i, m_{i}}=E\left[x_{i, m_{i}} \mid x_{i, o_{i}}, \theta\right]=\hat{\mu}_{m_{i}}+\hat{\Sigma}_{m_{i}, o_{i}} \hat{\Sigma}_{o_{i}, o_{i}}^{-1}\left(x_{i, o_{i}}-\hat{\mu}_{o_{i}}\right)
$$

Conforme James Honaker et. al. (2011), o algoritmo funciona bem mesmo com a utilização de outras distribuições ([HKB11]). Chien-Fu, mostra que a convergência do algoritmo EM está estabelecida pelo menos para um mínimo local, dando consistência a aplicabilidade dessa estratégia ([Wu70]).

\subsection{Sumarização}

A sumarização envolve métodos para descrever estatisticamente os dados utilizando medidas de posição como a média e a mediana, medidas de variabilidade como o desvio padrão e o coeficiente de variação e medidas de assimetria e curtose para analisar a distribuição dos dados ([HK06], [TSK06], [LL14] e [dSPB16]). O interesse principal, é descobrir alguns padrões antes da etapa de mineração e, por esse motivo, alguns autores definem essa etapa como Mineração Primitiva ([HK06], p. 31), pois permite que o usuário saiba quais perguntas devem ser feitas com base no conjunto de dados.

Além dessas medidas de interessabilidade, gráficos como box-plot e Scatter-plot foram aplicados para verificar a distribuição dos dados e analisar as correlações entre as variáveis, auxiliando de maneira ilustrativa na compreensão dos atributos e também nas medidas utilizadas (posição, variabilidade, assimetria e curtose) ([LL14] e [JBBA09]). 


\subsection{Análise de Predição (Classificação e Regressão)}

Classificação é o processo de encontrar um modelo ou função, $h: \mathcal{X} \rightarrow \mathcal{Y}$, que mapeia os dados $\mathcal{X}$ para suas classes correspondentes $\mathcal{Y}$, com o objetivo de predizer a classe de um objeto cujo rótulo não é conhecido. Para o mesmo objetivo, porém considerando os rótulos no conjunto dos números reais, a tarefa de regressão é estabelecida ([SSBD14] e [dSPB16]). Essa busca por um modelo utiliza um conjunto de treinamento ou uma amostra inicial com os rótulos, gerando uma função hipótese que depende fortemente da distribuição da amostra. Um exemplo didático sobre conjunto de treinamento, função hipótese e dependência em relação a distribuição da amostra é apresentado por Shai Shalev-Shwartz et al. ([SSBD14], p. 13) e por Mello e Ponti ([dMP18]).

A tarefa de predição pode ser aplicada em diversos problemas atuais, principalmente na área da saúde. Por exemplo, podemos usar a tarefa de classificação para predizer se um paciente está contaminado ou não por uma doença, a depender dos seus sintomas. Já a tarefa de regressão, pode ser utilizada como forma de estimar a altura e peso de um bebê recém-nascido, com base nas suas características durante a ultrassonografia.

Na teoria de Machine Learning um algoritmo de aprendizagem supervisionado procura por uma função $h$ no qual utiliza um conjunto de treinamento, $S=\left\{\left(\mathbf{x}_{1}, \mathbf{y}_{1}\right),\left(\mathbf{x}_{2}, \mathbf{y}_{2}\right), \ldots,\left(\mathbf{x}_{m}, \mathbf{y}_{m}\right)\right\} \subset \mathcal{X}$, que possibilita encontrar uma regra de predição ou modelo $h: \mathcal{X} \rightarrow \mathcal{Y}$ que possui o menor erro durante o treinamento, utilizando uma amostra de tamanho $m$ e no teste da regra. Nesse caso, o termo aprendizagem supervisionada pode ser entendio como um professor/supervisor que indica, durante o treinamento, quando o mapeamento $h\left(\mathbf{x}_{i}\right)=\mathbf{y}_{i}$ está certo, utilizando uma medida de penalização ou uma função de perda. Entretanto, um excelente modelo para a amostra não implica, necessariamente, que será bom durante o seu teste. Shai Shalev-Shwartz et al. ([SSBD14], p. 15) apresentam um contra-exemplo de uma hipótese que falha no teste mas que demonstrou ser um ótimo classificador durante o treinamento. Quando um algoritmo não utiliza as classes, $\mathcal{Y}$, para aprender, dizemos que o aprendizado é não-supervisionado.

Esses paradigmas de aprendizagem não são únicos. Marcelo K. Sanches ([San03]) apresenta de maneira sucinta cada tipo de paradigma e aprendizagem, abordando o estudo sobre aprendizado semi-supervisionado, onde parte dos rótulos são conhecidos.

Atualmente, existem várias técnicas utilizadas na tarefa de predição. Alguns exemplos são: SVM - Support Vector Machine, ELM - Extreme Learning Machine, Árvores de Decisão, Naive Bayes, KNN - Nearest Neighbor, MLP - Multilayers Perceptrons (Redes Neurais Artificiais), MMQ - Método dos Mínimos Quadrados para regressão linear e não linear, entre outros ([HTF01], [Alp04], [McC07], [Ize08], [Bar12] e [SSBD14]).

\subsection{Análise de Agrupamento}

Em MD a análise de agrupamento tem como objetivo identificar grupos de objetos que possuem características semelhantes entre si (homogeneidade) e diferentes dos outros objetos dos demais grupos (heterogeneidade). Existem várias aplicações, bem como na engenharia, ciências da compu- 
tação, medicina, biologia, astronomia, economia, ciências sociais, química, entre outras e está em constante desenvolvimento nos últimos anos, devido a grande complexidade nos dados e quantidade de informação disponível.

Obter um agrupamento num conjunto de dados de tamanho $N$ é extremamente trabalhoso, principalmente devido ao grande número de combinações possíveis. Liu ([Liu68], p. 39) apresenta a equação (2.4) que fornece o número de agrupamentos possíveis utilizando $N$ objetos para $k$ grupos. Por exemplo, se tomarmos $(N, k)=(30,3)$ teremos que $P(30,3) \approx 2 \times 10^{14}$ quantidade de grupos diferentes. Portanto, para que tenha aplicação prática é necessário que o número de grupos não seja muito grande.

$$
P(N, k)=\frac{1}{k !} \sum_{i=1}^{k}(-1)^{k-i}\left(\begin{array}{c}
k \\
i
\end{array}\right) i^{N} .
$$

O processo básico para a tarefa de agrupamento está ilustrado na Figura 2.4. A seleção ou extração permite identificar as características que mais discriminam os dados, para auxiliar o algoritmo na busca dos padrões reduzindo o tempo e dimensão do problema. A seleção ou criação do algoritmo está relacionada com a escolha da métrica de similaridade entre objetos, tipos de agrupamentos que serão usados e os tipos de abordagens dos algoritmos, tornando essa tarefa subjetiva. A validação dos agrupamentos está relacionada com a escolha das medidas de avaliação nos grupos gerados pelo algoritmo, o que permite uma análise sobre os resultados e possibilita alterar os parâmetros, a abordagem dos algoritmos e as métricas de proximidade para garantir maior confiabilidade nos resultados gerados. A última etapa trata-se da interpretação e compreensão dos resultados por meio dos gráficos e tabelas gerados pelo agrupamento em comparação com os dados originais.

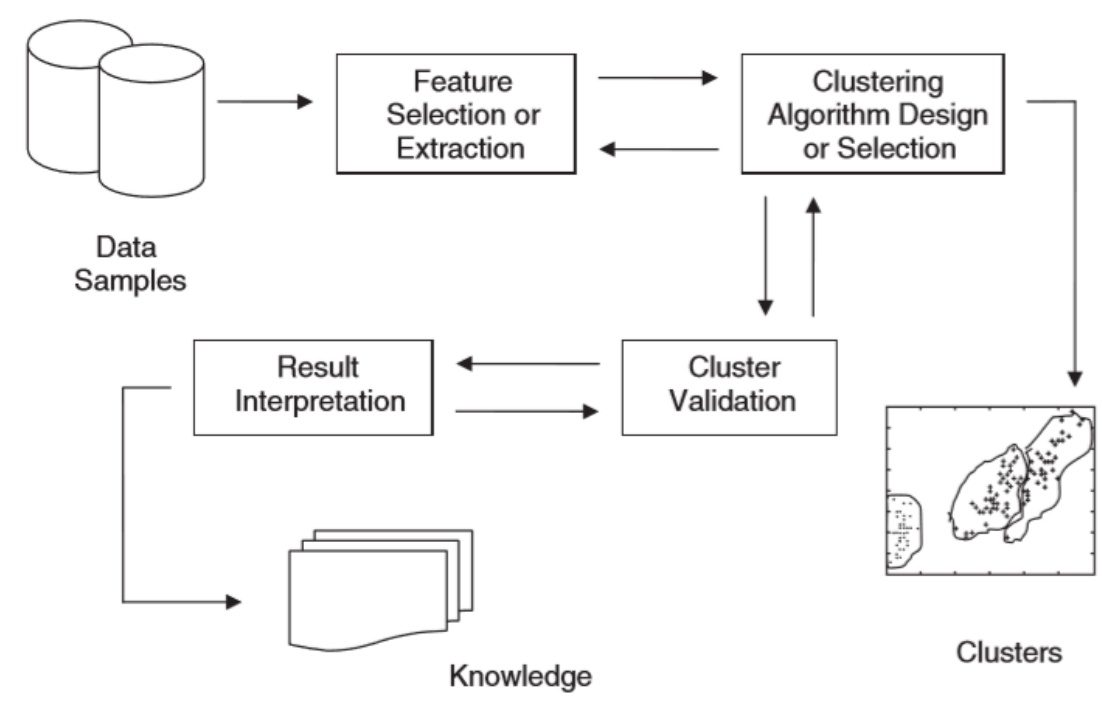

Figura 2.4: Processo básico da análise de agrupamento ([XW09], p. 6).

Os algoritmos de agrupamento são métodos não-supervisionados, ou seja, eles não utilizam os rótulos ou informações a priori sobre cada objeto em relação aos grupos que serão identificados. Existem, em geral, dois tipos de agrupamentos: particional e hierárquico: 
1. Particional: Gera $k$ grupos dos objetos de $\mathcal{X}, C=\left\{C_{1}, C_{2}, \ldots, C_{k}\right\}(k \leq N)$, tal que:

- $C_{i} \neq \emptyset, i \in 1, \ldots, k$;

- $\cup_{i=1}^{k} C_{i}=\mathcal{X}$;

- $C_{i} \cap C_{j}=\emptyset, i, j \in\{1, \ldots, k\}$ e $i \neq j$.

2. Hierárquico: Gera um dendrograma, isto é, um diagrama de árvore hierárquico, com uma estrutura particional aninhada de $\mathcal{X}, H=\left\{H_{1}, \ldots, H_{q}\right\}(q \leq N)$, tal que, se $C_{i} \in H_{m}, C_{j} \in H_{l}$ e $m \leq l$, então $C_{i} \subset C_{j}$ ou $C_{i} \cap C_{j}=\emptyset$, para quaisquer $i \in\left\{1, \ldots,\left|H_{m}\right|\right\}$ e $j \in\left\{1, \ldots,\left|H_{l}\right|\right\}$, com $m, l \in\{1, \ldots, q\}$.

A altura do dendrograma, que é a menor distância entre os grupos, está definida de baixo para cima, porém podemos defini-la de outra forma (de cima para baixo), alterando apenas a desigualdade de $m<l$ para $m>l$.

Cada técnica de agrupamento gera particionamentos do tipo:

- Exclusivo: cada objeto pertence apenas a um único grupo;

- Interseccionado: um objeto pode pertencer a dois grupos simultaneamente;

- Difuso: cada objeto tem um grau de pertinência em relação a todos os grupos.

Os tipos de grupos e suas técnicas de construção são abordados por Rui Xu et al. ([XW09]), Brian S. Everitt et al. ([ELLS11]) e Pang-Ning Tan et al. ([TSK06]). Pela definição de agrupamento particional, os grupos gerados são do tipo exclusivos, sendo assim, esses particionamentos são variações da definição $C_{i} \cap C_{j}=\emptyset$.

A questão fundamental na tarefa de agrupamento está na escolha da métrica entre os objetos, pois é necessário definir quão similares eles são. Em termos matemáticos, uma métrica é uma função $d: \mathcal{X} \times \mathcal{X} \rightarrow \mathbb{R}_{+}$, com $\mathcal{X} \subset \mathbb{R}^{d}$, tal que, para quaisquer valores de $\mathbf{x}, \mathbf{y}$ e $\mathbf{z} \in \mathcal{X}$ satisfaz as propriedades:

1. (Simetria) $d(\mathbf{x}, \mathbf{y})=d(\mathbf{y}, \mathbf{x})$;

2. (Reflexiva) $d(\mathbf{x}, \mathbf{x})=0$;

3. (Desigualdade Triangular) $d(\mathbf{x}, \mathbf{y}) \leq d(\mathbf{x}, \mathbf{z})+d(\mathbf{z}, \mathbf{y})$.

Definição 2.5.1 (Métrica de similaridade) Uma métrica de similaridade é uma função $s: \mathcal{X} \times \mathcal{X} \rightarrow$ $[0 ; 1]$, tal que, para quaisquer $\boldsymbol{x}, \boldsymbol{y} \in \mathcal{X}$, satisfaz:

- $s(x, x)=1$;

- $s(\boldsymbol{x}, \boldsymbol{y})=s(\boldsymbol{y}, \boldsymbol{x})$. 
A partir de uma função de similaridade, podemos obter diferentes funções de dissimilaridade, sendo a mais usual a identidade definida pela equação (2.5). As equações (2.6), (2.7) e (2.8) são outras formas de obtermos $s$ em termo de $d$ ([TSK06]).

$$
\begin{gathered}
d(\mathbf{x}, \mathbf{y})=\sqrt{1-s(\mathbf{x}, \mathbf{y})}, \forall \mathbf{x}, \mathbf{y} \in \mathcal{X} \\
s(\mathbf{x}, \mathbf{y})=\frac{1}{d(\mathbf{x}, \mathbf{y})+1}, \forall \mathbf{x}, \mathbf{y} \in \mathcal{X} \\
s(\mathbf{x}, \mathbf{y})=\exp (-d(\mathbf{x}, \mathbf{y})), \forall \mathbf{x}, \mathbf{y} \in \mathcal{X} ; \\
s(\mathbf{x}, \mathbf{y})=1-\frac{d(\mathbf{x}, \mathbf{y})-\min _{z \in \mathcal{X}} d(\mathbf{x}, \mathbf{z})}{\max _{z \in \mathcal{X}} d(\mathbf{x}, \mathbf{z})-\min _{z \in \mathcal{X}} d(\mathbf{x}, \mathbf{z})}, \forall \mathbf{x}, \mathbf{y} \in \mathcal{X} .
\end{gathered}
$$

As funções $d$ e $s$ são chamadas de métricas de proximidade e serão tratadas indistinguivelmente durante o trabalho. As métricas, em geral, dependem dos dados e do problema envolvido e, portanto, diferentes escolhas dessas funções implicam em diferentes grupos.

Alguns exemplos das métricas utilizadas na análise de agrupamento, são:

\section{Família de Minkowski}

Família de distâncias no espaço $L^{p}$, também chamado de distância p-norma, que para cada $\mathbf{x}, \mathbf{y} \in \mathcal{X} \subset \mathbb{R}^{d}$, sendo $d$ o número de variáveis, calcula a distância utilizando a equação (2.9):

$$
d(\mathbf{x}, \mathbf{y})=\left(\sum_{i=1}^{d}\left|x_{i}-y_{i}\right|^{p}\right)^{\frac{1}{p}} .
$$

Essa distância gera métricas de proximidade conhecidas como Manhattan $(p=1)$, Euclideana $(p=2)$ e Chebyshev $(p=\infty)$, conforme mostram as equações $(2.10),(2.11)$ e $(2.12)$, respectivamente. Essas métricas são sensíveis a ruídos e outliers, por isso, é importante aplicar um pré-processamento.

\section{Manhattan (City-block) ou distância 1-norma:}

$$
d(\mathbf{x}, \mathbf{y})=\sum_{i=1}^{d}\left|x_{i}-y_{i}\right| .
$$

Usada geralmente para comparar atributos quantitativos discretos, mas pode ser usada para comparar atributos quantitativos contínuos.

\section{Euclidiana ou distância 2-norma:}

$$
d(\mathbf{x}, \mathbf{y})=\left(\sum_{i=1}^{d}\left|x_{i}-y_{i}\right|^{2}\right)^{1 / 2}
$$

Aplicado geralmente, quando dois vetores são quantitativos contínuos. 


\section{Chebyshev ou distância $\infty$-norma:}

$$
d(\mathbf{x}, \mathbf{y})=\max _{i \in\{1, \ldots, d\}}\left|x_{i}-y_{i}\right|
$$

\section{Correlações}

Em algumas aplicações, como agrupamento de séries temporais ou análise de multicolinearidade, é necessário aplicar uma distância que indique o grau de correlação entre dois objetos ou variáveis. As métricas de proximidades definidas pelas equações (2.13) e (2.14), são usualmente aplicadas nesses casos. No entanto, estas métricas são incapazes de fornecer informações sobre a magnitude das diferenças de duas séries temporais, sendo aplicadas somente para diferenciar a forma ([XW09], p. 25).

$$
\begin{gathered}
d(\mathbf{x}, \mathbf{y})=\frac{1-r(\mathbf{x}, \mathbf{y})}{2} \\
d(\mathbf{x}, \mathbf{y})=1-\left|r_{s}(\mathbf{x}, \mathbf{y})\right|
\end{gathered}
$$

Na equação (2.14) a função $(\mathbf{x}, \mathbf{y}) \mapsto r_{s}(\mathbf{x}, \mathbf{y})$ é a correlação de Spearman obtida ranqueando os vetores $\mathbf{x}$ e $\mathbf{y}$ e aplicando a correlação linear de Pearson (equação (2.15)). Essa métrica indica a existência de uma correlação entre dois objetos, independente se a correlação é positiva ou negativa.

A função $(\mathbf{x}, \mathbf{y}) \mapsto r(\mathbf{x}, \mathbf{y})$ é a correlação amostral de Pearson entre dois vetores, definida pela equação (2.15), cujo os valores $\overline{\mathbf{x}}$ e $\overline{\mathbf{y}}$ são as médias amostrais dos vetores $\mathbf{x}$ e $\mathbf{y}$ pertencentes ao espaço Euclideano de dimensão $d$, respectivamente.

$$
r(\mathbf{x}, \mathbf{y})=\frac{\sum_{i=1}^{d}\left(x_{i}-\overline{\mathbf{x}}\right)\left(y_{i}-\overline{\mathbf{y}}\right)}{\sqrt{\sum_{i=1}^{d}\left(x_{i}-\overline{\mathbf{x}}\right)^{2} \sum_{i=1}^{d}\left(y_{i}-\overline{\mathbf{y}}\right)^{2}}} .
$$

Essas métricas são discutidas nos trabalhos de Pedro L. P. Silva ([dS16]) e Aretha B. Alencar ([Ale07]). Há, também, trabalhos como de Michel Marie Deza e Elena Deza ([DD16]) que traz uma enciclopédia de métricas que são usadas nas mais diversas áreas da ciência.

Ahlame D. Chouakria e Panduranga N. Nagabhushan (2007), propuseram uma métrica capaz de capturar a similaridade na forma de duas séries temporais através da equação (2.16)([CN07]). A função definida pela equação (2.17) é a correlação temporal de primeira ordem de duas séries $\mathbf{x}$ e $\mathbf{y}$ com imagem no intervalo $[-1 ; 1]$, onde 1 indica correlação positiva entre as diferenças de primeira ordem das duas séries, -1 a correlação negativa e 0 indica que não há correlação, ou que as séries não são monótonas nas taxas de crescimento/decrescimento.

$$
\begin{gathered}
d(\mathbf{x}, \mathbf{y})=\phi_{k}\left(r_{t}(\mathbf{x}, \mathbf{y})\right) \delta(\mathbf{x}, \mathbf{y}) \\
r_{t}(\mathbf{x}, \mathbf{y})=\frac{\sum_{i=1}^{d-1}\left(x_{i+1}-x_{i}\right)\left(y_{i+1}-y_{i}\right)}{\sqrt{\sum_{i=1}^{d-1}\left(x_{i+1}-x_{i}\right)^{2} \sum_{i=1}^{d-1}\left(y_{i+1}-y_{i}\right)^{2}}} .
\end{gathered}
$$

A função $\phi_{k}$, definida pela equação (2.18), é uma função de ajuste adaptativa com parâmetro 
$k \geq 0$ controlador dos pesos sobre a função $\delta$ que calcula a distância Euclideana entre as duas séries. Além da distância Euclideana, outras distâncias podem ser usadas na função $\delta$, como a distância de Frechet ([MV14], p. 5) ou da família Minkowski.

$$
\phi_{k}(u)=\frac{2}{1+e^{k u}} .
$$

Os algoritmos de agrupamento são organizados quanto aos tipo de abordagens que eles estão baseados. Suas classificações são:

- Protótipos ou centróides: O método cria uma partição inicial utilizando elementos que estão no espaço dos dados para, em seguida, aplicar uma técnica de realocação iterativa que tenta melhorar o particionamento;

- Hierárquicos: Cria uma decomposição hierárquica de um conjunto de dados. Os métodos hierárquicos podem ser aglomerativos ou divisivos, dependendo de como a decomposição hierárquica é formada - juntando decomposições ou dividindo composições, respectivamente. A cada passo, divisões ou junções são feitas;

- Baseados em densidade: A formação do grupo ocorre com base na densidade dos dados;

- Baseados em modelos: Criam uma hipótese sobre um modelo para cada um dos grupos e encontram o melhor ajuste dos dados ao modelo;

- Baseados em grids: Esses métodos quantizam o espaço dos dados em um número finito de células que forma uma estrutura em grid.

A Figura 2.5 mostra uma exemplificação dos diferentes tipos de abordagens na análise de agrupamento e alguns exemplos de algoritmos.

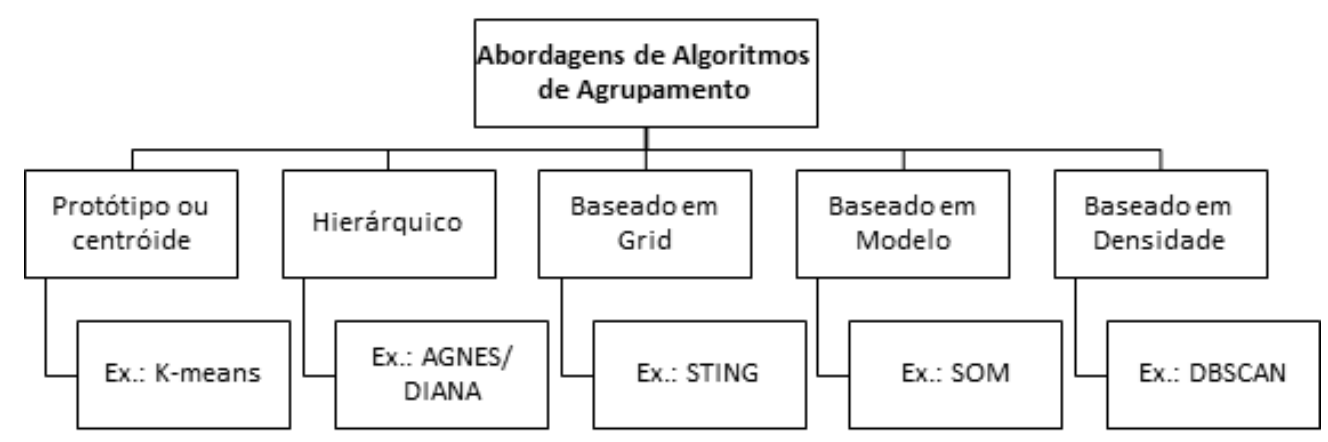

Figura 2.5: Tipos de abordagens dos algoritmos para análise de agrupamento.

Em geral, os algoritmos de agrupamento trabalham utilizando diretamente os dados. Entretanto, existem alguns que utilizam apenas a matriz de proximidade, definida em 2.5.2. 
Definição 2.5.2 (Matriz de Proximidade) Uma matriz $\boldsymbol{D} \in \mathbb{R}^{N, N}$, tal que $D_{i, j}=d\left(\boldsymbol{x}_{i}, \boldsymbol{x}_{j}\right)$, com $\boldsymbol{x}_{i}, \boldsymbol{x}_{j} \in \mathcal{X}$ é chamada de matriz de proximidade.

Neste trabalho, será utilizado um agrupamento hierárquico exclusivo. Para esse tipo de estratégia, o algoritmo mais utilizado na literatura é o AGNES - AGglomerative NESting ([Alp04], [HK06], [XW09], [Ize08], [ELLS11] e [dSPB16]).

O algoritmo AGNES utiliza uma abordagem aglomerativa, também conhecida como bottom-up que inicia com cada objeto formando um grupo separado e mescla, sucessivamente os objetos ou grupos próximos uns dos outros, até que todos os grupos sejam mesclados em um único grupo (o nível mais alto da hierarquia) ou até que uma condição de finalização seja atingida.

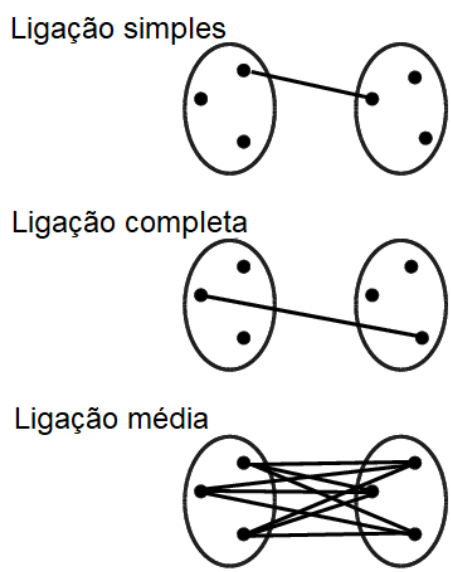

Figura 2.6: Métricas de uniões de grupos utilizados na análise de agrupamento hierárquico.

Além da métrica utilizada para comparar a proximidade entre os objetos, os algoritmos de agrupamento hierárquico utilizam uma métrica para comparar a proximidade entre dois grupos. Existem diversas métricas que podem ser utilizadas e elas são abordadas detalhadamente por Rui Xu e Donald C. Wunsch ([XW09], p. 31). Três dessas métricas são de grande importância em análise de agrupamento. Elas são definidas como single-linkage (ligação simples, ou vizinho mais próximo), complete-linkage (ligação completa, ou vizinho mais distante) e average-linkage (ligação médias, ou média das distâncias entre os grupos). Estas métricas estão representadas pelas equações (2.19), (2.20) e (2.21), respectivamente, e ilustradas na Figura 2.6.

$$
\begin{aligned}
& d_{I J, K}=\min _{K \neq I, J}\left\{d_{I, K}, d_{J, K}\right\} . \\
& d_{I J, K}=\max _{K \neq I, J}\left\{d_{I, K}, d_{J, K}\right\} . \\
& d_{I J, K}=\frac{\sum_{x \in C_{I J}} \sum_{y \in C_{K}} d(x, y)}{N_{I J} N_{K}} .
\end{aligned}
$$

De modo que, $C_{I J}$ é o grupo obtido pela união dos grupos $C_{I} \operatorname{com} C_{J}, N_{I J}$ é o número de elementos do grupo $C_{I J}, N_{K}$ é o número de elementos do grupo $C_{K}$ e $d_{J, K}$ (ou $d_{I, K}$ ) é a menor distância entre os grupos $C_{J}$ e $C_{K}$ (ou $C_{I}$ e $C_{K}$ ). O Algoritmo 1 mostra a abordagem hierárquica 
do AGNES, conforme Alan J. Izenman ([Ize08], p. 415).

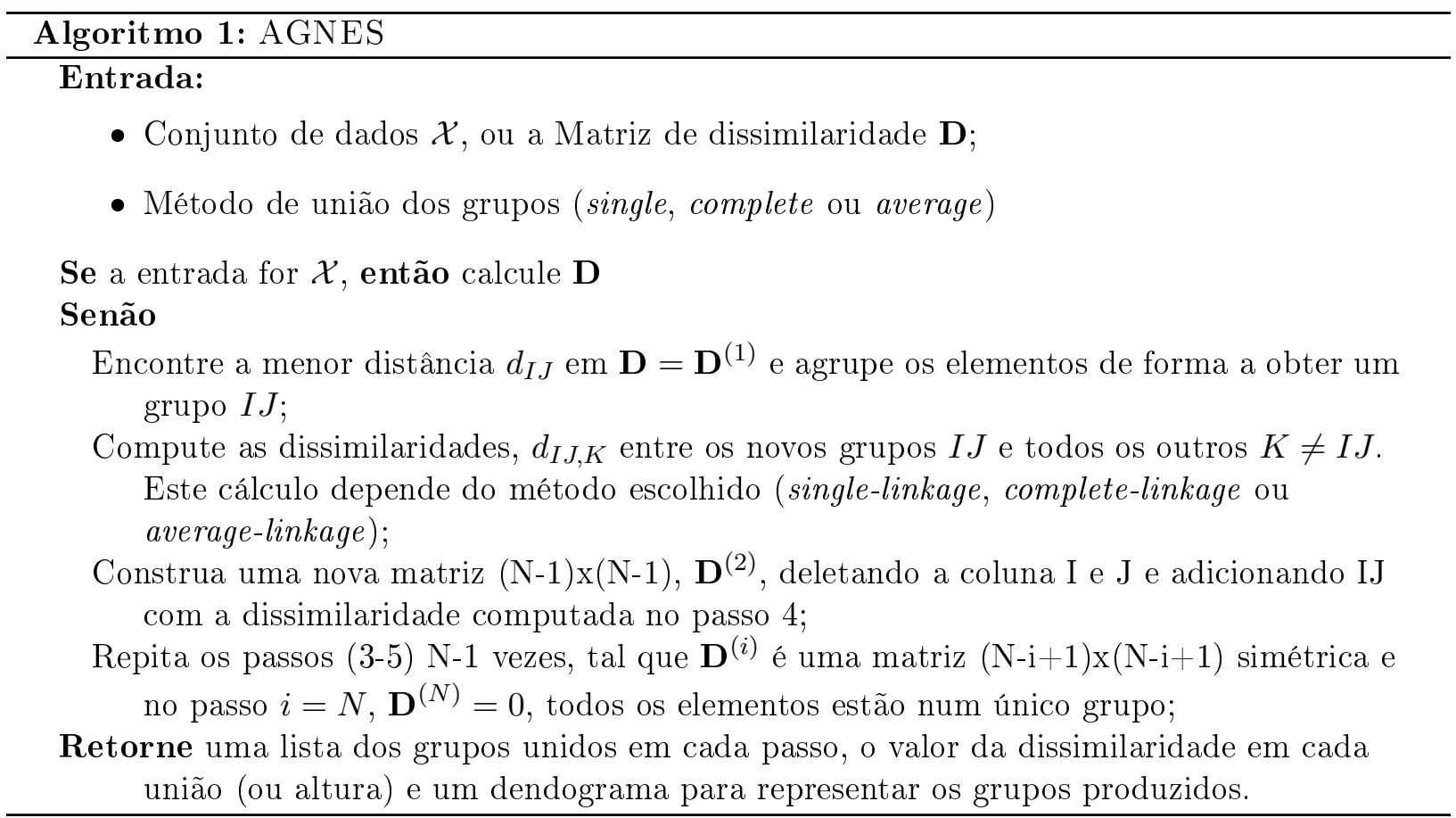

Esse algoritmo foi escolhido, pois permite mudar as métricas de proximidade sem alterar o algoritmo de agrupamento e não requer a definição do número de grupos a priori. As principais desvantagens estão na complexidade do tempo que, dependendo da métrica, pode chegar à ordem $\mathcal{O}\left(N^{2} \log N\right)$, sensibilidade a ruídos e outliers, dificuldade em encontrar grupos de diferentes formas e dimensões, falta da função objetivo e, alguns vezes, torna-se difícil identificar grupos visualmente a partir do dendograma.

\subsubsection{Validação dos Agrupamentos}

Diferentes grupos são gerados, dependendo da escolha do algoritmo de agrupamento ou mesmo da variação de parâmetros em um mesmo algoritmo. Sendo assim, não existe um único conjunto de grupos. Entretanto, é possível avaliar quantitativamente os diferentes resultados ([XW09]).

Há três abordagens para validação dos agrupamentos:

- Critérios Externos: Avalia os resultados com base numa estrutura pré-especificada, imposta ao conjunto de dados, ou seja, utiliza uma informação que não está presente no conjunto de dados;

- Critérios Internos: Avalia os resultados com base nos próprios dados de modo quantitativo;

- Critérios Relativos: Avalia os resultados por comparação com outros esquemas de agrupamentos resultantes de outras execuções do mesmo algoritmo, mas com parâmetros valorados de diferentes formas.

Os Critérios Externos são utilizados geralmente para validar algoritmos de agrupamentos, pois tem-se os rótulos dos grupos. Quanto aos Critérios Internos, há diversos índices sugeridos na lite- 
ratura ([XW09] e [ELLS11]). Nesta dissertação, optou-se pelos índices de Silhouette e coeficiente de correlação cophenético por serem usualmente utilizados em análise de agrupamentos hierárquicos.

O Índice de Silhouette foi introduzido por Rousseeuw em 1987 como forma de avaliar os grupos obtidos pelos algoritmos de agrupamentos ([Rou87]). Esse índice está definido para cada elemento $\mathrm{x} \in \mathcal{X}$. Considere $a_{i}$ como sendo a distância média do dado $\mathbf{x}_{i}$ a todos os elementos pertencentes ao mesmo grupo e $b_{i}$ a menor distância média do dado $\mathbf{x}_{i}$ a todos os demais grupos, excluindo o próprio grupo dele. Em termos matemáticos, temos que para todo $i=1, \ldots, N$ seja $\mathbf{x}_{i} \in C_{p}$ para algum $p \in\{1, \ldots, K\}$, sendo $K$ o número de grupos. Então as equações (2.22) e (2.23) definem os valores de $a_{i}$ e $b_{i}$, respectivamente.

$$
\begin{gathered}
a\left(\mathbf{x}_{i}\right)=a_{i}=\frac{\sum_{\mathbf{x}_{i} \neq \mathbf{x}, \mathbf{x} \in C_{p}} d\left(\mathbf{x}_{i}, \mathbf{x}\right)}{\left|C_{p}\right|-1} . \\
b\left(\mathbf{x}_{i}\right)=b_{i}=\min _{q=1, \ldots, K, q \neq p} \frac{\sum_{\mathbf{x} \in C_{q}} d\left(\mathbf{x}_{i}, \mathbf{x}\right)}{\left|C_{q}\right|} .
\end{gathered}
$$

O valor do Silhouette do objeto $\mathbf{x}_{i}$ no grupo $p, s_{i p}$, é dado pela equação (2.24), de modo que $-1 \leq s_{i p} \leq 1$, para todo $i \in\{1, \ldots, N\}$ e $p \in\{1, \ldots, K\}$. Um valore positivo de $s_{i p}$ (isto é, $a_{i} \approx 0$ ) indica que o i-ésimo objeto está bem agrupado, enquanto que um valor negativo de $s_{i p}$ (isto é, $\left.b_{i} \approx 0\right)$ indica que o i-ésimo objeto pode não estar bem agrupado e se $s_{i p}$ for próximo de zero, então o i-ésimo objeto está entre dois grupos. Se $\max _{\mathbf{x}_{i} \in C_{p}} s_{i p}<0,25$, indica que não há grupos bem definidos nos dados ou que, mesmo se houver, o procedimento não o encontrou.

$$
s_{i}\left(C_{p}\right)=s_{i p}=\frac{b_{i}-a_{i}}{\max \left\{a_{i}, b_{i}\right\}} .
$$

A média dos índices de Silhouette de cada grupo, $\bar{s}_{p}, \operatorname{com} p \in\{1, \ldots, K\}$ possibilita, para alguns algoritmos, encontrar parâmetros, por exemplo, o valor de $K$ do K-means. Como diagnóstico de agrupamento é usual definir o coeficiente de Silhouette como sendo $S C=\max _{k=1, \ldots, K}\left\{\bar{s}_{k}\right\}$. A Tabela 2.1 fornece uma interpretação subjetiva dos valores de SC. A principal vantagem na utilização desse índice é que necessita apenas da matriz de dissimilaridade e o agrupamento realizado.

\begin{tabular}{c|c}
\hline \multicolumn{1}{c|}{$\mathrm{SC}$} & Interpretação \\
\hline \hline $1,00-0,71$ & Uma estrutura forte foi encontrada \\
$0,70-0,51$ & Uma estrutura razoável foi encontrada \\
$0,50-0,26$ & A estrutura é fraca e pode ser artificial \\
$\leq 0,25$ & Nenhuma estrutura substancial foi encontrada \\
\hline
\end{tabular}

Tabela 2.1: Tabela com os intervalos para SC e suas interpretações (adaptado de [Ize08]).

O coeficiente de correlação cophenético é utilizado para analisar o resultado de um agrupamento hierárquico, seja ele aglomerativo ou divisivo. Esse índice foi criado por Robert R. Sokal e F. James Rohlff em 1962 num trabalho titulado como The Comparison of Dendograms by objetive methods ([eFJR62]). Esse índice depende da matriz cophenética, $\mathbf{H} \in \mathbb{R}^{N, N}$, criada através das alturas onde objetos foram unidos. Um exemplo didático para construir essa matriz foi apresentado por Everitt 
et al. ([ELLS11], p. 91).

Com o uso da matriz de dissimilaridade $\mathbf{D} \in \mathbb{R}^{N, N}$, sendo $N$ o número de objetos no conjunto de dados, o coeficiente de correlação cophenético, CPCC - Cophenetic Correlation Coefficient, é dada por:

$$
C P C C=\frac{\frac{1}{M} \sum_{i=1}^{N-1} \sum_{j=i+1}^{N} H_{i, j} D_{i, j}-\mu_{H} \mu_{D}}{\sqrt{\left(\frac{1}{M} \sum_{i=1}^{N-1} \sum_{j=i+1}^{N} H_{i, j}^{2}-\mu_{H}^{2}\right)\left(\frac{1}{M} \sum_{i=1}^{N-1} \sum_{j=i+1}^{N} D_{i, j}^{2}-\mu_{D}^{2}\right)}} .
$$

De modo que $\mu_{D}, \mu_{H}$ e $M$ são obtidos através das equações (2.26), (2.27) e (2.28), respectivamente.

$$
\begin{gathered}
\mu_{D}=\frac{1}{M} \sum_{i=1}^{N-1} \sum_{j=i+1}^{N} D_{i, j} . \\
\mu_{H}=\frac{1}{M} \sum_{i=1}^{N-1} \sum_{j=i+1}^{N} H_{i, j} . \\
M=\frac{N(N-1)}{2} .
\end{gathered}
$$

O coeficiente de correlação cophenético $C P C C$ pertence ao intervalo $[-1 ; 1]$, onde valores próximos de 1 indicam uma correlação forte entre a matriz cophenética e a de dissimilaridade, ou seja, o algoritmo ajustou bem o dendograma ao conjunto de dados. Alguns autores indicam que um valor aceitável para esse índice deve ser maior que 0.8, porém existem conjuntos de dados que mesmo para um índice 0.6 o dendograma tem um bom desempenho em termos de separabilidade ([Met06], p. 47). Por ser um coeficiente de correlação, esse índice permite obter testes estatísticos e o intervalo de confiança, similarmente ao coeficiente de correlação de Pearson.

\subsection{Associação}

A tarefa de associação foi criada inicialmente para obter padrões frequentes em cestas de supermercado, conforme publicações por Agrawal et al. ([ASI93] e [ASI94]) e, por isso, existem autores que definem a análise de associação como Análise de Cestas de Mercado ([HTF01] e [TSK06]). As técnicas de associação são consideradas um grande avanço na tecnologia de MD ([HTF01], p. 490), pois podem ser aplicadas a dados, sequenciais, séries temporais, espaciais, relacionais e multirrelacionais ([AS95], [HK06] e [Piv06]). Dessa forma, ela torna-se promissora na busca por padrões nos dados epidemiológicos espaciais.

Seja $\mathcal{I}=\left\{I_{1}, I_{2}, \ldots, I_{m}\right\}$ um conjunto de itens literais com todas as categorias das variáveis, podendo ser binário dependendo de sua representação. Considere uma base de dados estruturada $\mathcal{X}=\left\{\mathbf{x}_{1}, \mathbf{x}_{2}, \ldots, \mathbf{x}_{N}\right\}$ com $N$ objetos ou transações indexadas por um identificador $I D$, onde cada $\mathbf{x}_{k} \in \mathcal{X}$ é um conjunto de itens de $\mathcal{I}$, ou seja, $\mathbf{x}_{k} \subseteq \mathcal{I}$. A regra de associação é definida por: 
Definição 2.6.1 Uma regra de associação é uma implicação na forma $A \Longrightarrow B$, com $A, B \subset \mathcal{I}$ $e A \cap B=\emptyset$.

O conjunto de itens $A$ é chamado de antecedente, ou lado esquerdo, ou LHS (Left Hand Side), e o $B$ é o consequente, ou lado direito, ou RHS (Right Hand Side) da regra de associação $A \Longrightarrow B$. Neste trabalho, será considerado a forma compactada de representação dos itens na base de dados, ilustrada pelo exemplo na Figura 2.7b. Assim, cada linha representa um município e os itens são as categorias das variáveis envolvidas no problema. Existe outra forma de representação dessa base de dados, utilizada por alguns algoritmos de busca, chamada de forma binária (Figura 2.7a).

\begin{tabular}{|l|l|l|l|l|l|l|}
\hline $\mathrm{ID}$ & $I_{1}$ & $I_{2}$ & $I_{3}$ & $I_{4}$ & $I_{5}$ & $I_{6}$ \\
\hline 1 & 0 & 0 & 1 & 0 & 1 & 0 \\
\hline 2 & 1 & 1 & 0 & 1 & 1 & 0 \\
\hline 3 & 1 & 1 & 1 & 0 & 0 & 1 \\
\hline 4 & 1 & 1 & 1 & 0 & 1 & 0 \\
\hline 5 & 0 & 1 & 1 & 0 & 1 & 1 \\
\hline
\end{tabular}

(a) Base de dados binária.

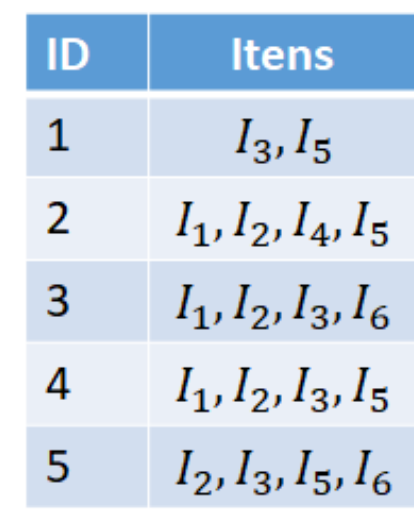

(b) Base de dados compactada.

Figura 2.7: Ilustração de duas bases de dados utilizadas no algoritmo de busca por regras de associação.

Considerando a Figura 2.7 como exemplo, com base nas definições dadas acima, temos que:

$$
\begin{aligned}
& \mathcal{X}=\left\{\left\{I_{3}, I_{5}\right\},\left\{I_{1}, I_{2}, I_{4}, I_{5}\right\},\left\{I_{1}, I_{2}, I_{3}, I_{6}\right\},\left\{I_{1}, I_{2}, I_{3}, I_{5}\right\},\left\{I_{2}, I_{3}, I_{5}, I_{6}\right\}\right\}, \text { com } N=5 ; \\
& \mathcal{I}=\left\{I_{1}, I_{2}, I_{3}, I_{4}, I_{5}, I_{6}\right\}
\end{aligned}
$$

Uma possível regra seria: $\left\{I_{2}, I_{3}\right\} \Longrightarrow\left\{I_{1}\right\}$, de modo que $\left\{I_{2}, I_{3}\right\} \cup\left\{I_{1}\right\}=\left\{I_{1}, I_{2}, I_{3}\right\}$ e $\left\{I_{2}, I_{3}\right\} \cap\left\{I_{1}\right\}=\emptyset$.

Dizemos que o objeto $\mathbf{x}_{k} \in \mathcal{X}$ contém o itemset $A$ se $A \subset \mathbf{x}_{k}$. Entende-se, naturalmente, por itemset como um conjunto de itens de $\mathcal{I}$. Considere a função $A \longmapsto \sigma(A)$ definida conforme a equação (2.29). Essa função indica a frequência do itemset $A$ no conjunto de dados, ou seja, o número de objetos no qual o itemset $A$ aparece.

$$
\sigma(A)=\left|\left\{\mathbf{x}_{i}: A \subset \mathbf{x}_{i}, \mathbf{x}_{i} \in \mathcal{X}\right\}\right| .
$$

Duas medidas de interesse (interestingness) que refletem a utilidade e a confiabilidade da regra é o suporte e a confiança, respectivamente. Elas são definidas como:

Definição 2.6.2 (Suporte e Confiança) Dado um conjunto $\mathcal{X}$ com $N$ objetos. O suporte e confi- 
ança de uma regra de associação $A \Longrightarrow B$ são definidos, respectivamente, por:

$$
\begin{gathered}
s(A \Longrightarrow B)=\frac{\sigma(A \cup B)}{N}, \\
c(A \Longrightarrow B)=\frac{\sigma(A \cup B)}{\sigma(A)} .
\end{gathered}
$$

Na definição acima, o suporte é a frequência relativa da união dos itens $A$ e $B$. O suporte de um itemset $A$, será denotado por $s(A)=\sigma(A) / N$. A confiança pode ser entendida como, dentre os objetos que contém $A$, a porcentagem de objetos que contém o $B$. Desta forma, a equação (2.32) mostra como a confiança pode ser escrita em função do suporte.

$$
c(A \Longrightarrow B)=\frac{s(A \cup B)}{s(A)} .
$$

Portanto, o problema de encontrar regras de associação pode ser resumido por ([TSK06]):

Dada uma base de dados estruturada $\mathcal{X}$, encontrar todas as regras que satisfazem $s(A \Longrightarrow B) \geq$ MinSup e $c(A \Longrightarrow B) \geq \operatorname{MinConf}$, sendo MinSup, MinConf $\in \mathbb{R}_{+}$, ou seja, o suporte mínimo e a confiança mínima, respectivamente, definidos pelo usuário.

Um conjunto de itens que satisfaz um suporte mínimo é dito frequente e uma regra de associação que satisfaz tanto um suporte mínimo quanto uma confiança mínima é dita forte. Portanto, um algoritmo de busca por regras de associação está delineado por dois parâmetros: MinSup e MinConf.

Este é um problema considerado difícil. A Figura 2.8 mostra abordagem à força-bruta, a qual computa todas as possíveis regras. Neste caso a complexidade do problema é na ordem de $\mathcal{O}(N M w)$, onde $N$ é o número de objetos, $w$ o comprimento do objeto com o maior número de itens e $M$ a quantidade de candidatos que podem ser formados pela regra, sendo proporcional a $2^{d}-1$, com $d=|\mathcal{I}|$, ou seja, o número total de itens distintos. A quantidade de regras que podem ser feitas com $d$ itens distintos, é dada pela expressão:

$$
R(d)=\sum_{i=1}^{d-1}\left[\left(\begin{array}{l}
d \\
i
\end{array}\right) \sum_{j=1}^{d-i}\left(\begin{array}{c}
d-i \\
j
\end{array}\right)\right] .
$$

Na sua forma simplificada, temos:

$$
R(d)=3^{d}-2^{d+1}+1 .
$$

Uma abordagem geralmente adotada para obter as regras de associação é dividir o algoritmo em duas etapas, sendo a primeira a busca por itens frequentes e a segunda na geração das regras de associação a partir dos itens frequentes obtidos.

Um dos primeiros algoritmos propostos para a tarefa de associação foi o APRIORI em 1994 por Rakesh Agrawal e Ramakrishnan Srikant ([ASI94]). Esse algoritmo utiliza o suporte mínimo e o Teorema 2.6.3 para reduzir a ordem de comparação $N M$. 


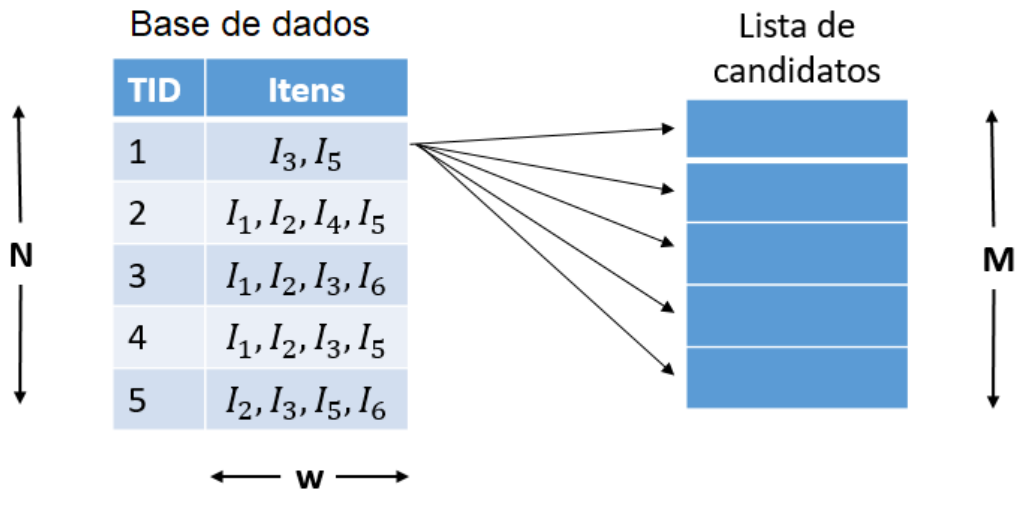

Figura 2.8: Ilustração da complexidade na busca por regras de associação.

Teorema 2.6.3 (Princípio Apriori) Se um itemset é frequente, então todos os seus subconjuntos também deve ser frequente.

O teorema sugere a busca por itens frequentes no conjunto de dados, pois a função a que define o suporte de um conjunto de itens é antimonotônica, ou seja, para quaisquer dois conjuntos de itens, $A, B \subset \mathcal{I}$, com $A \subset B$, tem-se $\sigma(A) \geq \sigma(B)$. Desta forma, se um subconjunto é infrequente, então todos seus superconjuntos também o serão (Figura 2.9).

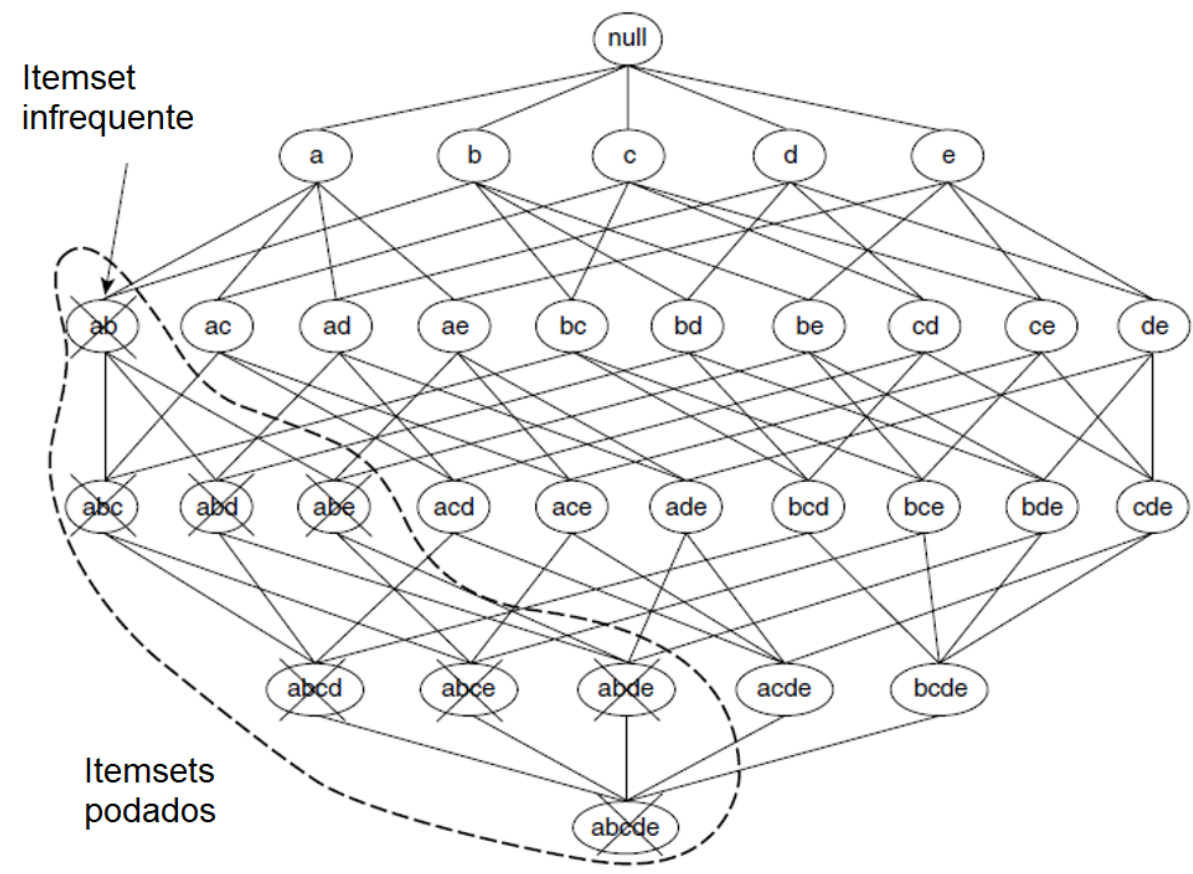

Figura 2.9: Poda dos itemsets infrequentes com base no Teorema 2.6.3 (Adaptado de Pang-Ning Tan et al. ([TSK06])).

O Algoritmo 2 que apresenta o APRIORI, utiliza os seguintes termos e notações:

- k-itemset: Conjunto com k itens; 
- $L_{k}$ : Conjunto com k-itemsets. Nesse conjunto há dois campos, sendo um para o itemset e o outro para a contagem do suporte;

- $C_{k}$ : Conjunto com os candidatos. Esse conjunto pode ser potencialmente grande, pois conterá as combinações possíveis dos itens.

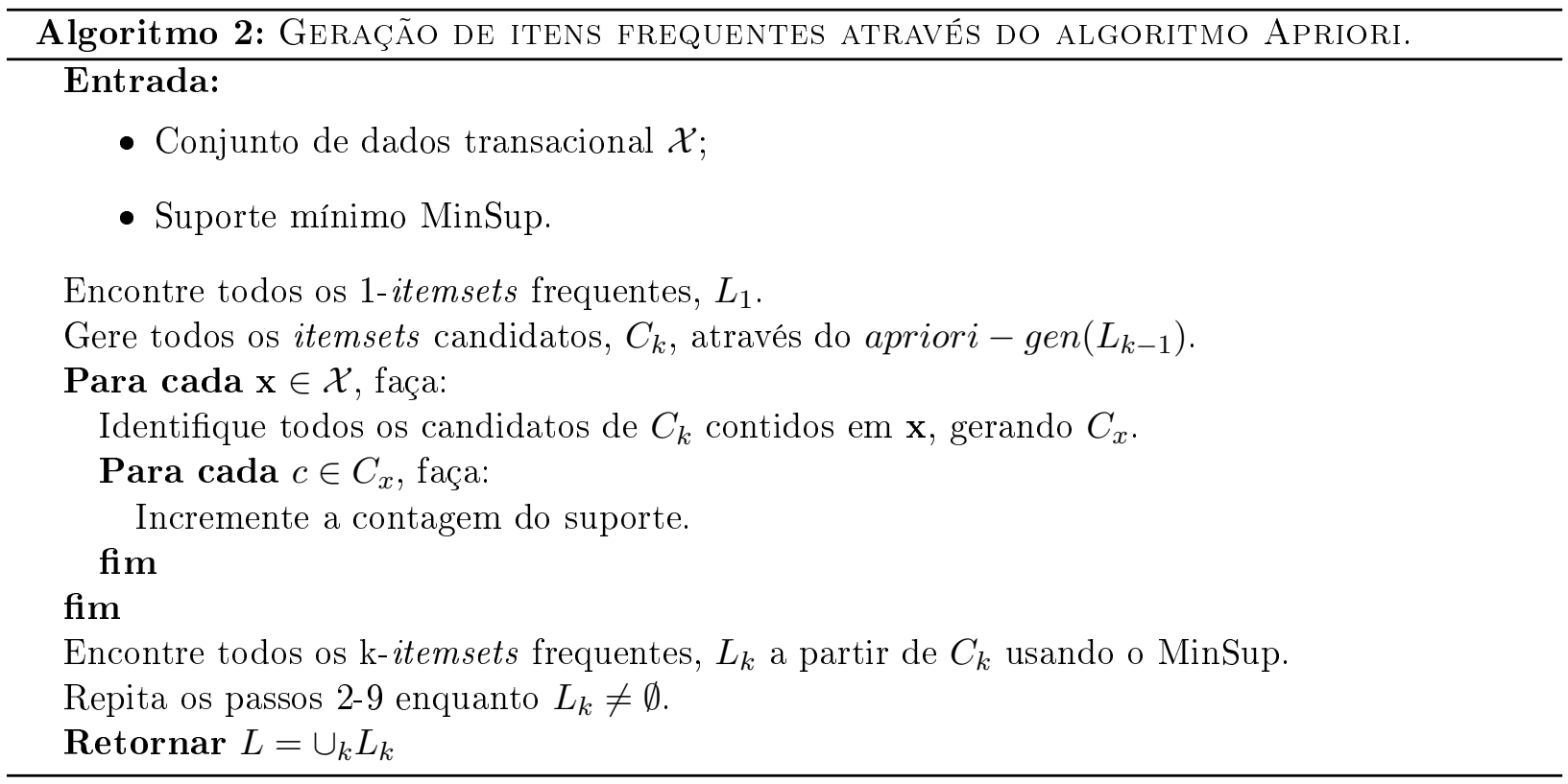

No Algoritmo 2, a função apriori - gen gera os candidatos pelas uniões dos elementos de $L_{k-1}$ com ele mesmo, ou seja, se dois (k-1)-itemsets $A, B \in L_{k-1}$ tiverem seus k-2 primeiros itens iguais, então ocorrerá a união, caso contrário, não ocorre a união. Após isso, uma varredura é feita para identificar se há algum subconjunto infrequente, realizando a poda dos itemsets infrequentes utilizando o Princípio Apriori.

A Figura 2.10 ilustra como funciona a varredura do algoritmo para a obtenção dos conjuntos com os itemsets frequentes utilizando a base de dados da Figura 2.7b. O conjunto $\left\{I_{1}, I_{2}, I_{3}\right\}$ com os candidatos de nível 3 já possui um suporte menor que 2, pois o conjunto $\left\{I_{1}, I_{3}\right\}$ não satisfaz o MinSup. Portanto, pelo Princípio Apriori para esse conjunto, também não irá satisfazer o MinSup.

Ao obtermos o conjunto dos itemsets frequentes a geração das regras também cresce exponencialmente. Dessa forma, o Teorema 2.6.4 permite contornar esse problema utilizando novamente o suporte para podar as regras não fortes.

Teorema 2.6.4 Considere um conjunto de itens frequentes $X$. Se uma regra $A \Longrightarrow X-A$ não satisfaz uma confiança mínima, então, qualquer regra $A^{\prime} \Longrightarrow X-A^{\prime}$, onde $A^{\prime} \subset A$, também não irá satisfazer a confiança mínima.

Demonstração. Suponha um conjunto $X$ de itens frequentes e um parâmetro MinConf $\in \mathbb{R}_{+}$ referente a confiança mínima. Considere duas regras de associação dadas por $A \Longrightarrow X-A$ e $A^{\prime} \Longrightarrow X-A^{\prime}$, onde $A^{\prime} \subset A$. Por hipótese, temos que a confiança da primeira regra não satisfaz 

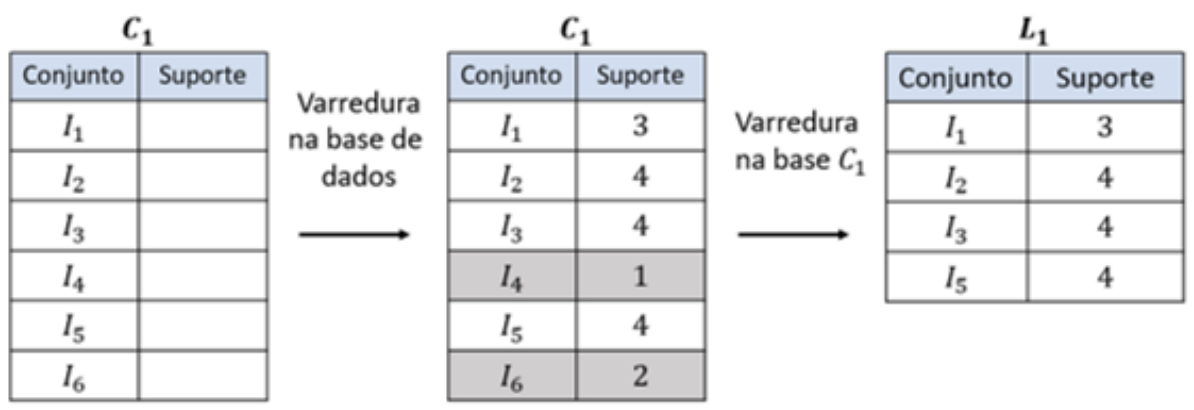

\begin{tabular}{|c|l|}
\multicolumn{2}{c}{$C_{2}$} \\
\hline Conjunto & Suporte \\
\hline$I_{1}, I_{2}$ & \\
\hline$I_{1}, I_{3}$ & \\
\hline$I_{1}, I_{5}$ & \\
\hline$I_{2}, I_{3}$ & \\
\hline$I_{3}, I_{5}$ & \\
\hline
\end{tabular}

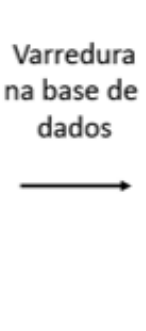

\begin{tabular}{|c|c|}
\multicolumn{2}{c}{$C_{2}$} \\
\hline Conjunto & Suporte \\
\hline$I_{1}, I_{2}$ & 3 \\
\hline$I_{1}, I_{3}$ & 2 \\
\hline$I_{1}, I_{5}$ & 2 \\
\hline$I_{2}, I_{3}$ & 3 \\
\hline$I_{3}, I_{5}$ & 3 \\
\hline
\end{tabular}

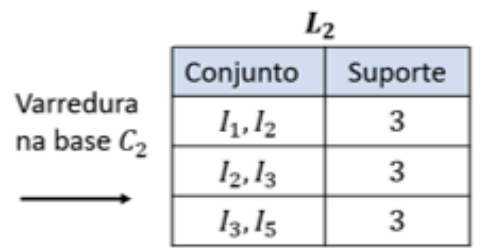

\begin{tabular}{|c|c|}
\multicolumn{1}{c}{$C_{3}$} \\
\hline Conjunto & Suporte \\
\hline$I_{1}, I_{2}, I_{3}$ & \\
\hline$I_{2}, I_{3}, I_{5}$ & \\
\hline
\end{tabular}
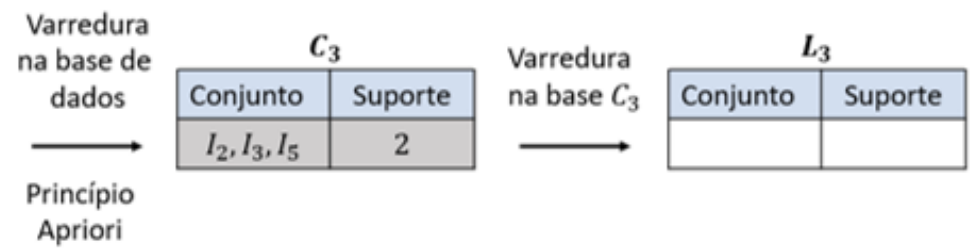

Figura 2.10: Ilustração da geração do itemset frequente utilizando o algoritmo APRIORI para um MinSup $>2$.

uma confiança mínima, ou seja,

$$
c(A \Longrightarrow X-A)=\frac{s(X)}{s(A)}<\operatorname{MinConf} .
$$

Como a função suporte é antimonotônica, temos também que $s\left(A^{\prime}\right) \geq s(A)$, pois $A^{\prime} \subset A$. Assim,

$$
\frac{1}{s\left(A^{\prime}\right)} \leq \frac{1}{s(A)}
$$

Logo,

$$
c\left(A^{\prime} \Longrightarrow X-A^{\prime}\right)=\frac{s(X)}{s\left(A^{\prime}\right)} \leq \frac{s(X)}{s(A)}<\operatorname{MinConf}
$$

O Algoritmo 3, descreve como é feita a busca por regras de associação fortes.

As desvantagens na utilização desse algoritmo é que ele trabalha apenas com valores categóricos e isso traz uma perda de informação, pois é necessário categorizar os dados numéricos. Outra desvantagem é que o algoritmo não faz a busca temporal, ou seja, regras de associação para as quais o tempo é levado em consideração não são buscadas. Essa segunda desvantagem é tratada em alguns trabalhos como de Mohammed J. Zaki ([Zak01]) num algoritmo chamado de SPADE - An Efficient 


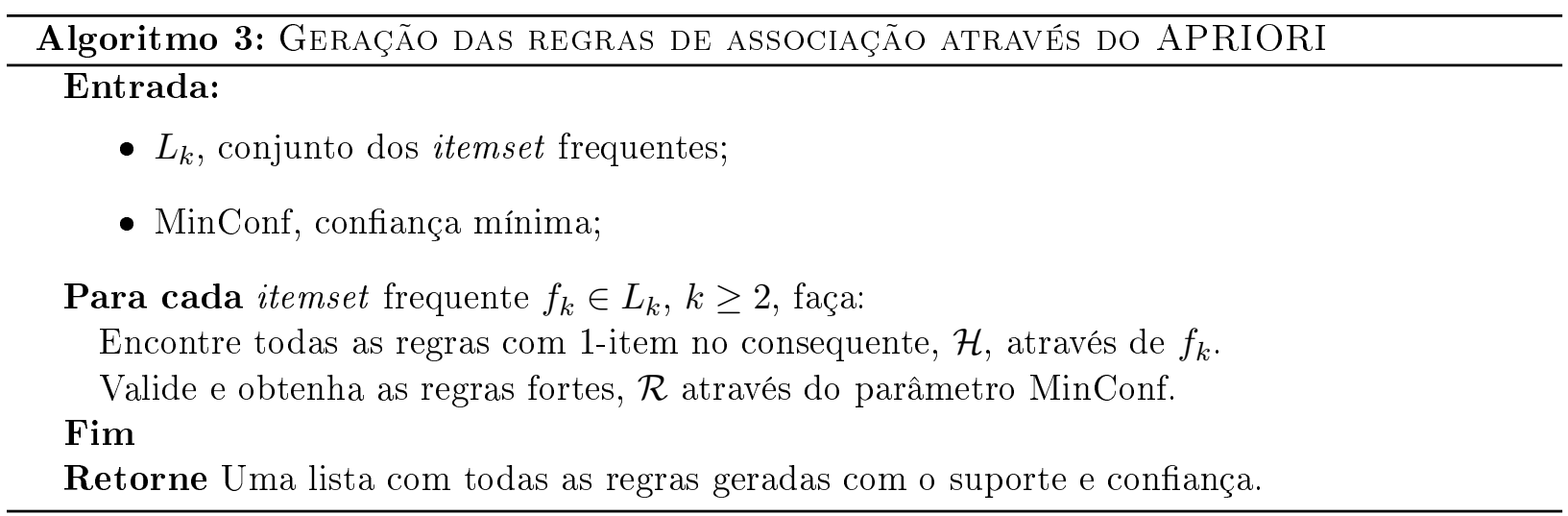

Algorithm for Mining Frequent Sequence e no trabalho Marcos D. Cano ([Can12]) que implementa um algoritmo chamado de SART para buscar regras de associação entre séries temporais.

As avaliações das regras são feitas por medidas de interesse como o suporte e a confiança. Se o suporte é relativamente baixo, ocorrerão muitas regras de associação acarretando um alto custo computacional e, além disso, regras não interessantes aparecerão com mais frequência. Para um suporte relativamente alto, teríamos uma menor quantidade de regras, porém os itens raros não seriam identificados.

Algoritmos mais sofisticados foram propostos para contornar o problema dos itens raros ou esporádicos que, por definição, possuem suporte muito baixo, mas são importantes para o analista ([KR05]). Trabalho como de Liu et al. ([LHM99]) permite buscar as regras utilizando múltiplos suportes mínimos através de um novo algoritmo chamado MSApriori. Neste trabalho, não será abordado a busca por itens raros.

\subsubsection{Avaliações das Regras de Associação}

Uma regra é dita interessante quando satisfaz as medidas de interesse (critérios objetivos) e é importante para o usuário (critérios subjetivos). Pang-Ning Tan et al. ([TKS04]) indica critérios objetivos que podem ser utilizados na análise de associação. Em geral, esses critérios procuram analisar a independência entre LHS e RHS e a força da regra. Além do suporte e confiança, critérios como Lift, Rule Interest e Convição serão utilizados neste trabalho para identificar as regras interessantes. Esses três índices são abordados e exemplificados no trabalho de Eduardo C. Gonçalves ([Gon05]) e foram escolhidos, pois traduzem de diferentes formas a independência do consequente para o antecedente.

Definição 2.6.5 (Independência entre itens) Numa regra de associação $A \Longrightarrow B$, com $A \cap B=\emptyset$, $o$ itemset $B$ é independente de $A$ se:

$$
s(A \cup B)=s(A) s(B) .
$$

O termo $s(A \cup B)$ é chamado de suporte real da regra, já o termo $s(A) s(B)$ é o suporte espe- 
rado. Caso não ocorra a igualdade, dizemos que existe uma dependência entre o antecedente e o consequente da regra. Neste caso, há duas possibilidades:

Dependência positiva:

$$
s(A \cup B)>s(A) s(B) .
$$

Dependência negativa:

$$
s(A \cup B)<s(A) s(B) .
$$

A dependência positiva indica que a força da regra está no sentido do antecedente para o consequente, i.e., a ocorrência do LHS aumenta a probabilidade de RHS. Para a dependência negativa, a força da regra está no sentido contrário da regra, i.e., a ocorrência LHS diminui a probabilidade de RHS.

O Lift é definido como:

$$
l(A \Longrightarrow B)=\frac{c(A \Longrightarrow B)}{s(B)} .
$$

Substituindo a equação (2.32) em (2.40), ela pode ser reescrita como:

$$
l(A \Longrightarrow B)=\frac{s(A \cup B)}{s(A) s(B)} .
$$

Essa medida trata-se de uma estimativa de independência do antecedente e do consequente da regra $A \Longrightarrow B$, pois pela definição 2.6.5, se $l(A \Longrightarrow B)=1$, temos que $s(A \cup B)=s(A) s(B)$ indicando que os itens são independentes.

O índice RI - Rule Interest (regra interessante) introduzido por Piatetsky-Shapiro em 1991 ([PS91]) é definido como:

$$
R I=s(A \Longrightarrow B)-s(A) s(B) .
$$

Esse parâmetro pertence ao intervalo $[-0,25 ; 0,25]$, de modo que, se $R I=0$, então o suporte encontrado é igual ao suporte esperado, ou seja, $A$ e $B$ são independentes. Caso $R I>0$, então $A$ e $B$ são positivamente dependentes. Se $R I<0$, então $A$ e $B$ são negativamente dependentes. Quanto maior for $|R I|$, mais interessante será a regra.

A Convicção foi proposta em 1997 e tem objetivo analisar a independência de uma regra de associação, porém, a análise é baseada na lógica proposicional, ou seja, na tabela verdade para a implicação $A \Longrightarrow B$ na sua forma equivalente a $\sim A \vee B$. Essa última expressão pode ser reescrita como $\sim(A \wedge \sim B)$ e, em termos do suporte, é definido pela equação (2.43).

$$
\operatorname{Conv}(A \Longrightarrow B)=\frac{s(A) s(\bar{B})}{s(A \cup \bar{B})} .
$$

Esse índice pertence ao intervalo $[0 ; \infty]$, onde valores próximo de 1 indicam independência completa entre o RHS e o LHS e valores altos indicam que o antecedente nunca aparece sem o consequente. A equação (2.44) permite obter a conviç̧ão em termos do suporte do consequente e 
da confiança da regra, facilitando o processo computacional.

$$
\operatorname{Conv}(A \Longrightarrow B)=\frac{1-s(B)}{1-c(A \Longrightarrow B)}
$$

\subsection{Análise de Dados Espaciais}

A análise de dados espaciais é usualmente empregada em dados econométricos e ecológicos, a fim de modelar problemas envolvendo dependências espaciais ([BPGR08] e [Alm12]). Esse termo tem uma linhagem na geografia a partir do trabalho de Patrick Alfred Pierce Moran (1917 - 1988) sobre problemas de dependência espacial da fertilidade do solo ([Mor50]). Neste trabalho, para a análise da dependência espacial entre as variáveis municipais estudadas, utilizamos o índice local, global e o diagrama de Moran.

Considere um vetor ou variável $\left(x\left(s_{1}\right), x\left(s_{2}\right), \ldots, x\left(s_{N}\right)\right)^{T}$, onde cada elemento $x\left(s_{i}\right)$ está indexado por sua localização espacial $s_{i}$. Neste trabalho cada índice $i$ representa a localização do centro de um dos municípios do estado de São Paulo, ou seja, sua latitude e longitude. O conjunto de dados espaciais utilizado é do tipo polígono, ou seja, cada observação $x_{i}$ está contida numa área poligonal. Existem outros tipos de dados espaciais, tais como pontos, linhas ou grids, conforme Roger S. Bivand et al. ([BPGR08]).

Para analisar a dependência espacial em todo o estado para cada variável foi utilizado o Índice Global de Moran (IGM), definido pela equação (2.46). Esse índice identifica a covariância entre os valores dos municípios e de seus vizinhos mais próximos. Devido a normalização $I \in[-1 ; 1]$, onde valores próximos de 1 indicam correlação espacial positiva, ou seja, municípios com altos valores e similares ao seus vizinhos, -1 correlação espacial negativa e 0 não possui dependência espacial. Para obter esse índice utiliza-se a matriz $\mathbf{W} \in \mathbb{R}^{N, N}$ normalizada de proximidade espacial. Essa matriz também é chamada de matriz de Markov ou linhas estocásticas, visto que possui as seguintes propriedades:

- $\sum_{j=1}^{N} W_{i, j}=1$, para todo $i$, ou seja, a soma de cada linha é igual a um;

- $W_{i, j} \geq 0$ para todo $i$ e $j$ de 1 até $N$.

A matriz $\mathbf{W}=\mathbf{D}^{-1} \mathbf{B}$, em que $\mathbf{B} \in \mathbb{R}^{N, N}$, é a matriz simétrica construída conforme a equação (2.45) e $\mathbf{D} \in \mathbb{R}^{N, N}$ é uma matriz diagonal, de forma que cada elemento na diagonal principal é a soma da i-ésima linha de $\mathbf{B}$.

$$
\begin{gathered}
B_{i, j}=\left\{\begin{array}{l}
1, \text { se a observação j é vizinho de i } \\
0, \text { c.c. }
\end{array}\right. \\
I=\frac{N \sum_{i=1}^{N} \sum_{j=1}^{N} W_{i, j}\left(x_{i}-\overline{\mathbf{x}}\right)\left(x_{j}-\overline{\mathbf{x}}\right)}{\left(\sum_{i=1}^{N} \sum_{j=1}^{N} W_{i, j}\right)\left(\sum_{i=1}^{N}\left(x_{i}-\overline{\mathbf{x}}\right)^{2}\right)} .
\end{gathered}
$$

A Figura 2.11 mostra o estado de São Paulo com todas as conexões construídas a partir da distância entre cada município e seus vizinhos com o uso da matriz B. A Figura 2.12 representa 
a quantidade de municípios comparada com o número de vizinhos imediatamente próximos, também chamados de 1-vizinhos ou vizinho de primeira ordem, gerados a partir dessas conexões. Cerca de 149 municípios apresentam 5 vizinhos de primeira ordem indicando uma característica do espaço.

Conexão Espacial no Estado de São Paulo

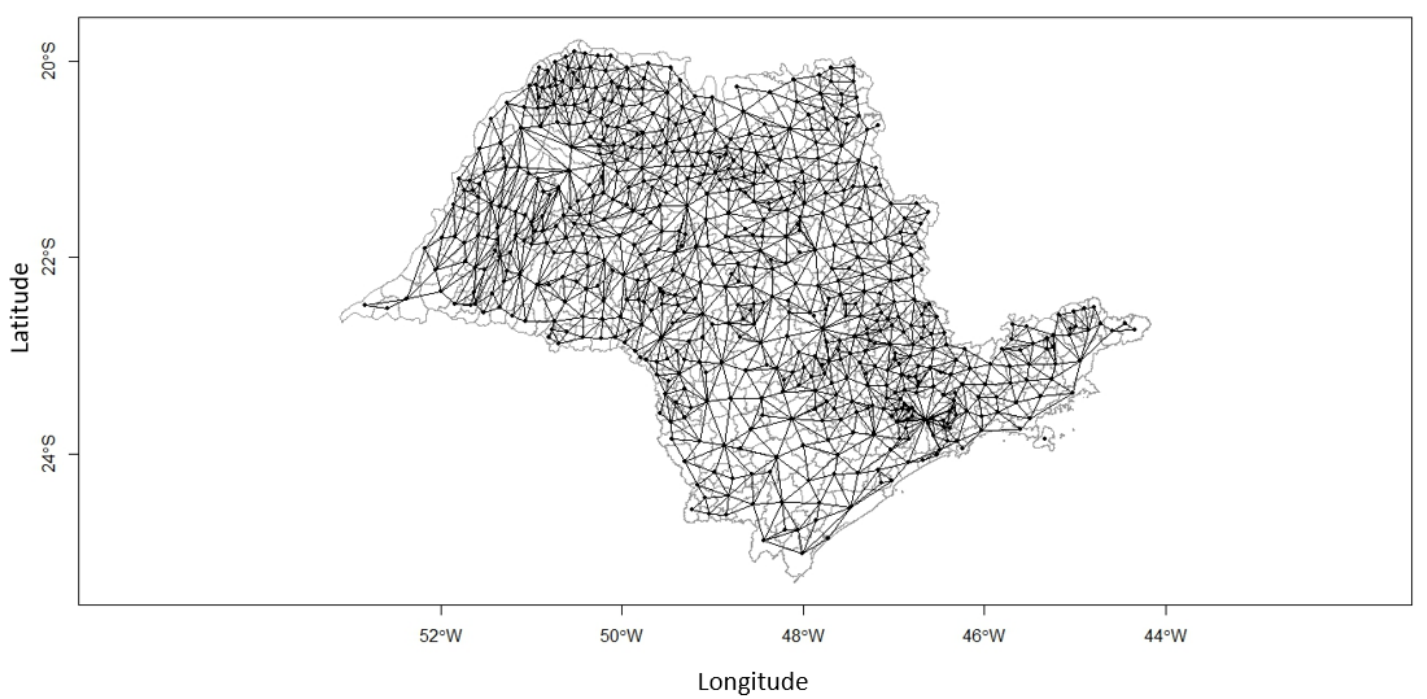

Figura 2.11: Conexões espaciais existentes nos municípios do estado de São Paulo.

Distribuição dos Municípios pelo Número de Vizinhos

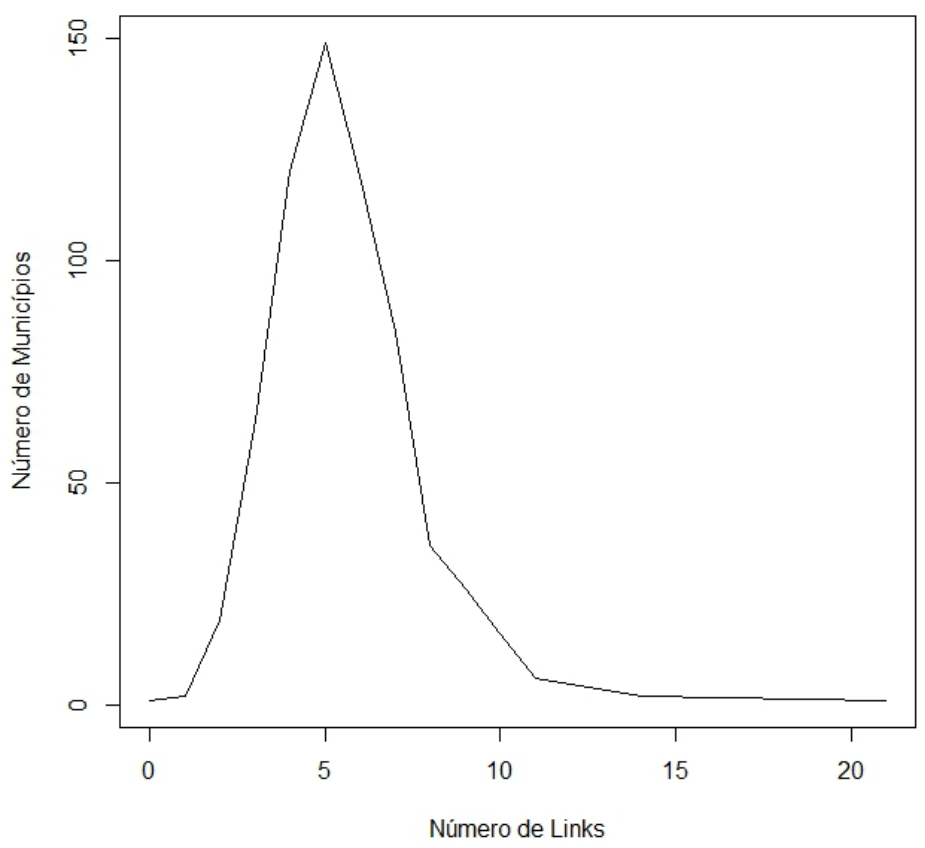

Figura 2.12: Distribuição dos municípios em relação ao número de links (ou vizinhos próximos) no estado de São Paulo.

Na equação (2.46) $N=645$, que corresponde ao total de municípios. O vetor $\mathbf{x} \in \mathbb{R}^{N}$ representa a variável a qual estamos analisando a dependência espacial. A forma matricial do IGM é dada pela 
equação (2.47).

$$
I=\frac{(\mathbf{x}-\overline{\mathbf{x}})^{T} \mathbf{W}(\mathbf{x}-\overline{\mathbf{x}})}{(\mathbf{x}-\overline{\mathbf{x}})^{T}(\mathbf{x}-\overline{\mathbf{x}})} .
$$

Os testes estatísticos desse índice podem ser obtidos de duas maneiras:

- Pseudossignificância: obtido fazendo diversas permutações dos municípios, em geral é denotado por 999 permutações, conforme Antônio et al. ([LnFR17]). Como cada permutação produz um novo arranjo espacial, pode ser construída uma distribuição empírica dos diversos índices obtidos na qual apenas uma corresponde à situação observada. Caso essa seja um valor extremo da distribuição, então, existe significância estatística já que trata-se de um valor atípico dentre todas simuladas.

- Comparação com a distribuição normal padrão: normaliza o índice global utilizando a equação (2.48) e, com isso, utiliza-se da distribuição normal padrão para obter o p-valor com algum nível de significância. Isso porque, $I^{*} \sim \mathcal{N}(0,1)$ conforme indica Philip Viton $(2010$, p. 8) e Roger S. Bivand et al. (2013, p. 278).

$$
I^{*}=\frac{I-E[I]}{\sqrt{V[I]}} .
$$

Para ambos os casos, a hipótese nula e alternativa que serão testadas são dadas por:

- $H_{0}: I=0$ (não há autocorrelação espacial entre os municípios);

- $H_{a}: I>0$ (existe autocorrelação espacial positiva entre os municípios).

Na equação (2.48), o valor esperado $E[I]$ e a variância $V[I]$ são obtidos como:

$$
\begin{gathered}
E[I]=\frac{-1}{N-1}, \\
V[I]=\frac{N S_{4}-S_{3} S_{1}(1-2 N)}{(N-1)(N-2)(N-3)\left(\sum_{i=1}^{N} \sum_{j=1}^{N} W_{i, j}\right)^{2}} .
\end{gathered}
$$

Em que,

$$
\begin{gathered}
S_{1}=\frac{1}{2} \sum_{i=1}^{N} \sum_{j=1}^{N}\left(W_{i, j}+W_{j, i}\right)^{2}, \\
S_{2}=\sum_{i=1}^{N}\left(\sum_{j=1}^{N} W_{i, j}+W_{j, i}\right)^{2}, \\
S_{3}=\frac{N \sum_{i=1}^{N}\left(x_{i}-\overline{\mathbf{x}}\right)^{4}}{\left(\sum_{i=1}^{N}\left(x_{i}-\overline{\mathbf{x}}\right)^{2}\right)^{2}} \\
S_{4}=\left(N^{2}-3 N+3\right) S_{1}-N S_{2}+3\left(\sum_{i=1}^{N} \sum_{j=1}^{N} W_{i, j}\right)^{2} .
\end{gathered}
$$


A Figura 2.13 ilustra a distribuição desses índices de Moran obtidos por 999 permutações dos municípios do estado de São Paulo para a variável incidência de tuberculose normalizada. Como o índice obtido está situado no extremo da distribuição, o valor é significativo ( $\mathrm{p}$-valor $=2,22 \times 10^{-11}$ ).

Gráfico da Densidade dos Índices permutados

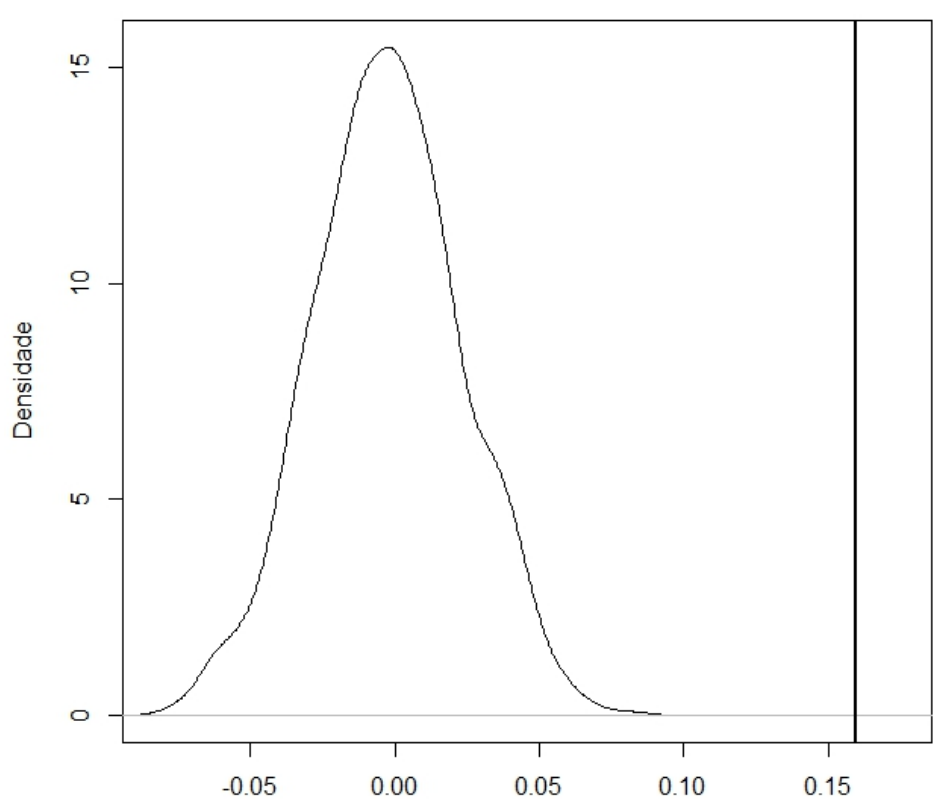

Log da Taxa de Incidência de Tb via simulação por Monte Carlo do I de Moran

Figura 2.13: Gráfico da densidade gerada pelo indice de Moran obtidos pelas diferentes permutações ocorridas nos municípios, referente ao logaritmo da incidência de tuberculose em 2010.

Para a análise espacial local, foi utilizado o Índice Local de Moran (ILM) definido pela equação (2.55). Diferente do índice global que caracteriza a região como um todo, o índice local permite identificar municípios que possuem altas correlações espaciais, sendo possível criar grupos locais de municípios que possuem o mesmo comportamento.

$$
I_{i}=\frac{N\left(x_{i}-\overline{\mathbf{x}}\right) \sum_{j=1}^{N} W_{i, j}\left(x_{j}-\overline{\mathbf{x}}\right)}{\sum_{j=1}^{N}\left(x_{j}-\overline{\mathbf{x}}\right)^{2}} .
$$

Esse índice pode ser reescrito na sua forma matricial conforme a equação (2.56). O vetor $\mathbf{I} \in \mathbb{R}^{N}$, possui o índice local para cada município $i$. Uma relação importante entre o índice Global e Local de Moran é dada pela equação (2.57).

$$
\begin{gathered}
\mathbf{I}=\operatorname{diag}\left(\frac{N(\mathbf{x}-\overline{\mathbf{x}})(\mathbf{x}-\overline{\mathbf{x}})^{T} \mathbf{W}^{T}}{(\mathbf{x}-\overline{\mathbf{x}})^{T}(\mathbf{x}-\overline{\mathbf{x}})}\right) . \\
I=\sum_{i=1}^{N} \frac{I_{i}}{N} .
\end{gathered}
$$

O valor descritivo do teste estatístico para o ILM pode ser obtido de maneira similar ao teste do índice global, alterando apenas a equação analítica da variância (eq. (2.58)) ([Ans95]). Após a normalização do índice local para cada localidade $i$, pela equação (2.48), utiliza-se a distribuição 
normal padrão para o índice normalizado, dado algum nível de significância.

$$
V\left[I_{i}\right]=\frac{\left(N-b_{2}\right) \sum_{j=1}^{N} W_{i, j}^{2}}{N-1}+\frac{\left(2 b_{2}-N\right) \sum_{k=1}^{N} \sum_{h=1}^{N} W_{i, k} W_{j, h}}{(N-1)(N-2)}-\frac{1}{N-1} .
$$

Em que,

$$
b_{2}=\frac{\sum_{i=1}^{N}\left(x_{i}-\overline{\mathbf{x}}\right)^{4}}{\left(\sum_{i=1}^{N}\left(x_{i}-\overline{\mathbf{x}}\right)^{2}\right)^{2}} .
$$

Neste trabalho, o nível de significância foi definido em 5\%, similarmente aos trabalhos sobre análise epidemiológica como feito por Alexandre S. P. Siqueira ([Siq14]), Alessandra G. L. Pereira et al. ([PdAME $\left.\left.{ }^{+} 15\right]\right)$ e Antônio J. R. Luzardo et al. ([LnFR17]).

O diagrama de espalhamento de Moran é uma metodologia aplicada para identificar grupos locais e categorizar os dados numéricos com base em seus valores e localização espacial. Esse diagrama utiliza a variável normalizada e a média dos vizinhos também normalizado, descritos pelas equações (2.60) e (2.61), respectivamente. O diagrama de Moran é gerado pelo conjunto $\Omega$ (equação (2.62)) no plano cartesiano.

$$
\begin{gathered}
\mathbf{z}=\frac{(\mathbf{x}-\overline{\mathbf{x}})}{s d[\mathbf{x}]} . \\
\mathbf{z}_{v i z}=\mathbf{W} \mathbf{z} . \\
\Omega=\left\{\left(z_{i}, z_{v i z, i}\right) \mid i=1,2, \ldots, N\right\} .
\end{gathered}
$$

Os quadrantes $Q m, m=1,2,3,4$, definem as possíveis associações entre os municípios e seus vizinhos, como descrito a seguir:

- Q1 (valores positivos e médias positivas) e Q2 (valores negativos e médias negativas): indicam pontos de associação espacial positiva, ou seja, são municípios cujo seus vizinhos possuem valores semelhantes;

- Q3 (valores positivos e médias negativas) e Q4 (valores negativos e médias positivas): indicam pontos de associação espacial negativa, ou seja, são municípios cujo seus vizinhos não possuem valores semelhantes ou distintos.

A Figura 2.14 representa os quadrantes mencionados acima. Importante salientar que, por definição, municípios que não possuem vizinhos de primeira ordem tem índice local nulo, esse é o caso da Ilha Bela localizada no litoral do estado de São Paulo.

O Algoritmo 4 foi usado para obter os índices locais de Moran, o valor descritivo e a categorização de cada município, em relação aos quadrantes do diagrama de Moran utilizando as equações anteriores. 


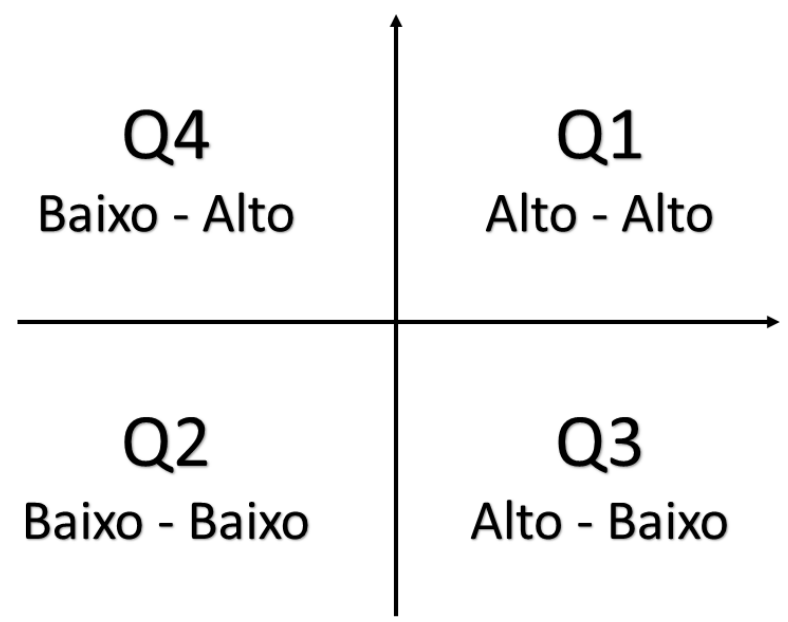

Figura 2.14: Ilustração dos quadrantes utilizados pelo Diagrama de Moran.

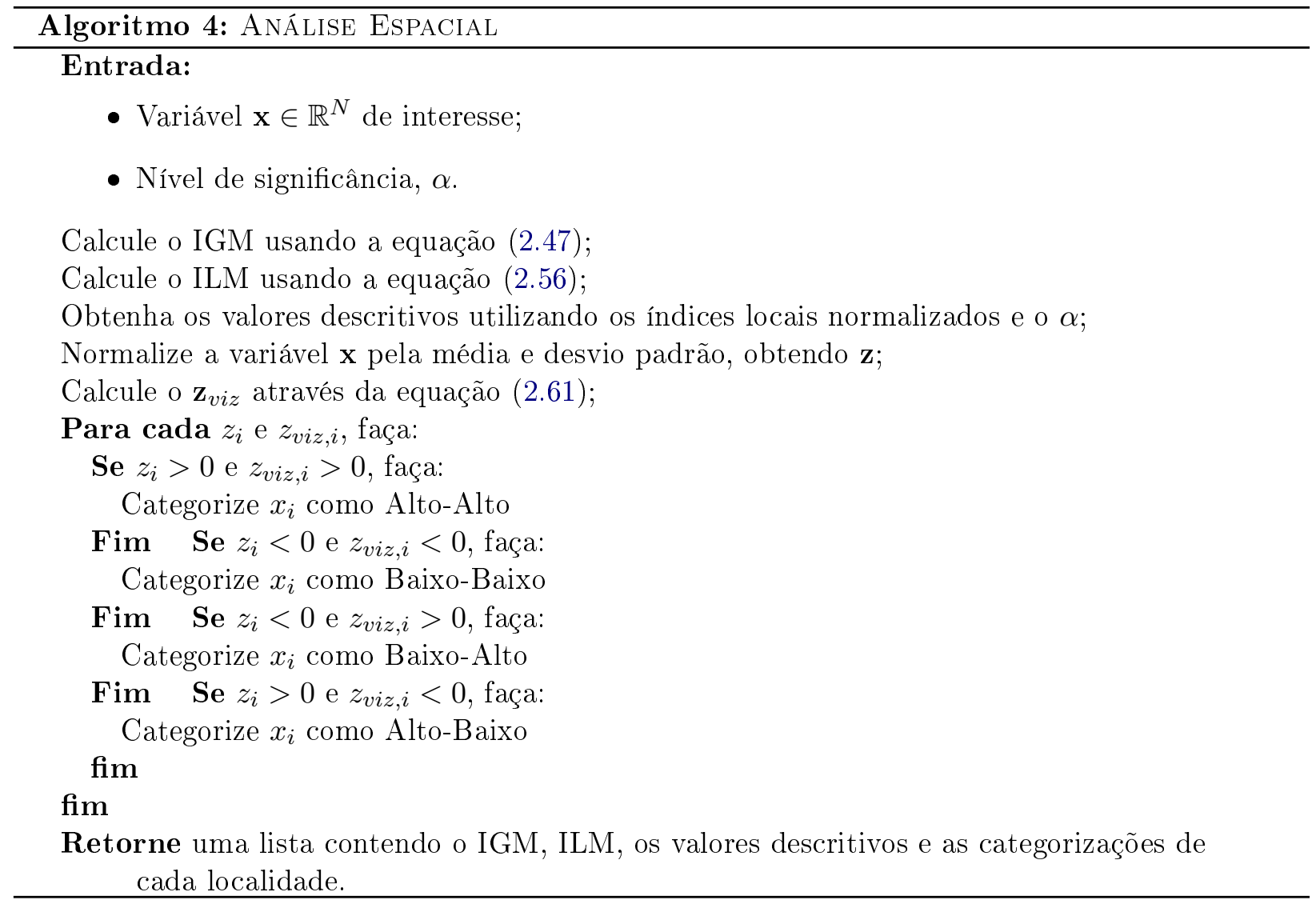

O pacote SPDEP do R, criado por Roger Bivand et al. ([BPGR08]) possui os algoritmos e estatísticas de dependências espaciais que foram utilizados neste trabalho. Vale lembrar que existem outros pacotes disponíveis em outras linguagens e programas que buscam por padrões espacias. 


\section{Capítulo 3}

\section{Materiais e Metodologia}

\subsection{Materiais}

Neste trabalho, os dados utilizados referem-se aos municípios do estado de São Paulo e foram obtidos de três fontes principais: da Fundação de Sistema Estadual de Análise de Dados - SEADE (www.seade.gov.br); do Instituto Brasileiro de Geografia e Estatística - IBGE (www.ibge.gov.br); do Departamento de Informática do Sistema Único de Saúde - DATASUS (www.datasus.saude.gov.br).

Os conjuntos de dados socioeconômicos são estruturados com 645 instâncias representando os municípios. Quanto aos dados do DATASUS, o total de instâncias varia, pois dependem do número de casos notificações de cada município.

Essas informações foram separadas em duas categorias:

- Dados sobre a Doença: Informações sobre as notificações (data, município, evolução, casos em cada faixa etária, taxa de incidência, etc.). A incidência, prevalência e mortalidade, são indicadores epidemiológicos que podem ser incluídos nesta base de dados;

- Dados Socioeconômicos: Informações municipais tais como, despesas com saúde, educação, transporte, dados sobre violência (homicídios, presídios, etc.), abandono escolar, IDHM, entre outros. Nesta categoria é possível inserir outras bases de dados e concatená-las com os dados da doença. Nesta categoria é possível inserir outras bases de dados e concatená-las com os dados da doença.

A Figura 3.1 ilustra o fluxograma para a obtenção dos dados, identificando atributos, período selecionado e nomenclaturas, para todas as categorias dos dados socioeconômicos e da doença.

Todas as informações contidas em Dados sobre a Doença foram obtidas por meio do Departamento de Informática do SUS (DATASUS) e baixados em 12/2017 ([DAT18]). Com o intuito de estudar a incidência nos municípios no período de 2001 até 2017 , foram baixados os dados de casos confirmados por Tipo de Entrada do DATASUS, incluindo casos pulmonares e extrapulmonares, com e sem confirmação laboratorial e todas as categorias de cultura de escarro e testes com TB. Essas bases de dados possuem um conjunto de atributos específicos, que podem ser consultados no 


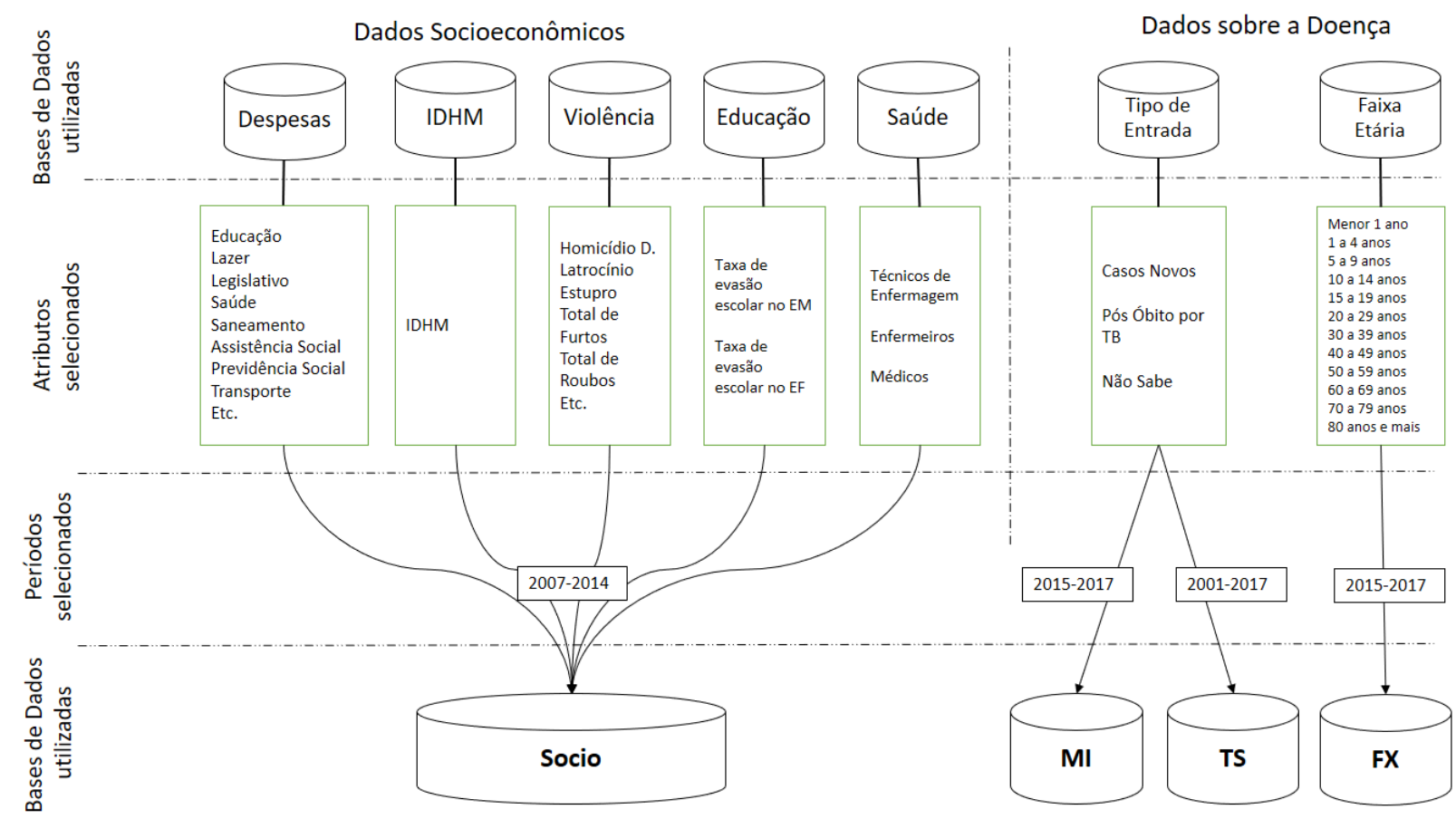

Figura 3.1: Fluxograma para a obtenção dos materiais que serão utilizados neste trabalho.

Dicionário de Dados na página do SINAN ([dNadAdNaS18]).

Para a base de dados Tipo de Entrada foram utilizados os seguintes atributos: Casos Novos, Pós-óbito por TB e Não Sabe para gerar as incidências da doença, conforme as orientações disponíveis no site do DATASUS TB ([DAT18]). A descrição destas variáveis estão na Tabela 3.1. A taxa de incidência de TB para cada município e ano é proporcional a 100 mil habitantes e foi calculada através da equação (3.1).

$$
\text { Taxa de Incidência }=\frac{\mathrm{N}^{\mathrm{o}} \text { de casos novos anuais no município }}{\text { População total no município }} \times 10^{5} .
$$

Foi definido TS a série temporal anual com as taxas de incidência para cada município no período de 2001 até 2017, a fim de analisar o comportamento da doença nesse período.

As taxas de incidência do período de 2001 a 2017 foram transformadas em um único valor para cada município, definido pela média aritmética das taxas dos 3 últimos anos (2015, 2016 e 2017). Outras possibilidades foram avaliadas, tais como a média aritmética e mediana de todos os dados disponíveis. Entretanto, estas se mostraram menos representativas que a média dos 3 últimos anos. Dessa forma, uma nova variável foi definida como MI - Média das taxas de Incidência.

A variável FX indica a faixa etária mais atingida da população municipal. Seu valor é calculado pelas médias das prevalências nos últimos 3 anos de cada faixa etária normalizada. A equação (3.2) apresenta a normalização, definida pelo número total de casos dividido pela população total em 


\begin{tabular}{c|l|l}
\hline Sigla & Variável & Significado \\
\hline \hline- & Casos Novos & $\begin{array}{l}\text { Nunca utilizou medicação antituberculosa ou a utilizou } \\
\text { por menos de 30 dias. }\end{array}$ \\
\hline- & Pós-óbito & $\begin{array}{l}\text { Caso não registrado no SINAN e que foi descoberto } \\
\text { ou notificado após a morte do paciente em decorrência da } \\
\text { realização de investigação epidemiológica. }\end{array}$ \\
\hline- & Não Sabe & $\begin{array}{l}\text { Caso com história prévia desconhecida. Deve ser registrado } \\
\text { apenas quando esgotadas as possibilidades de investigação } \\
\text { da história ou antecedente epidemiológico. }\end{array}$ \\
\hline
\end{tabular}

Tabela 3.1: Rótulos dos atributos da doença que foram utilizados para gerar a incidência de tuberculose, conforme regulamento no DATASUS.

cada faixa etária.

$$
T b_{N o r m}(i, k, t)=\frac{T b(i, k, t)}{\operatorname{Pop}(i, k, t)} .
$$

De modo que $T b_{N o r m}(i, k, t)$ e Pop $(i, k, t)$ significa o número de casos de tuberculose e a população total, respectivamente, na faixa etária $k$, no município $i$ e no ano $t$ variando de 2015 até 2017 .

Como as categorias das faixas etárias do IBGE são diferentes das definidas pelo DATASUS, foi necessário uma nova organização acumulando as notificações em 4 faixas etárias: de 0 até 9 anos, de 10 até 19 anos, de 20 até 59 e idade superior a 60 anos. Essas categorias foram denominadas como: Crianças, Adolescentes, Adultos e Idosos, respectivamente, baseando-se no estatuto da criança e adolescente ([BRA90]) e no estatuto do idoso ([BRA03]). A Figura 3.2 ilustra como estão organizadas as faixas etárias nos diferentes órgãos.

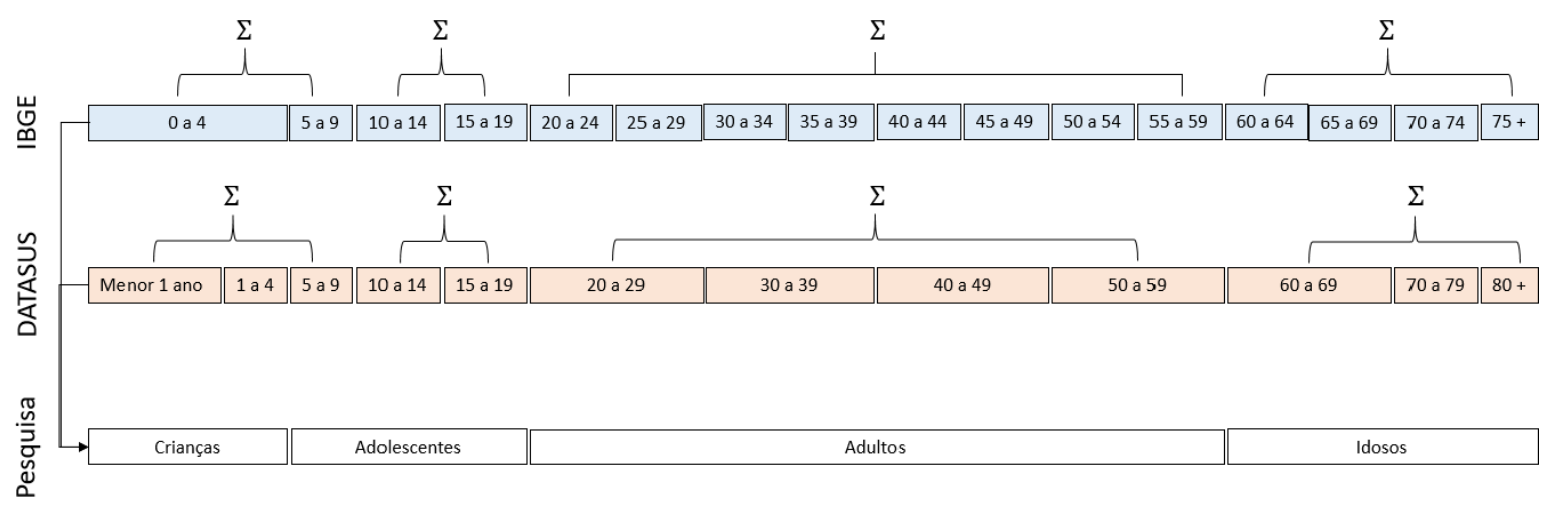

Figura 3.2: Metodologia para a redistribuição das taxas em cada faixa etária do IBGE e do DATASUS, com base nas categorias definidas pelo Estatuto da Criança e Adolescente e no Estatuto do Idoso.

Após a normalização, criou-se uma variável contendo as médias dessas prevalências nos últimos 3 anos em cada faixa etária, utilizando a equação (3.3):

$$
\bar{T} b_{N o r m}(i, k)=\frac{\sum_{t=2015}^{2017} T b_{N o r m}(i, k, t)}{3} .
$$

Como o objetivo é analisar a faixa etária mais atingida, foi extraído o argumento máximo desse 
vetor em cada município $i$ utilizando a equação (3.4):

$$
F X_{-} \max (i)=\arg \max _{k} \bar{T} b_{\text {Norm }}(i, k) .
$$

Em relação aos Dados Socioeconômicos, a Tabela 3.2 mostra as bases que serão utilizadas e a quantidade de atributos de cada uma delas, os períodos disponíveis e as suas respectivas referências. Em relação ao período, as bases são todas anuais. O formato desses arquivos estão em CSV Comma Separated Values.

\begin{tabular}{l|c|l|l}
\hline Nome da Base de Dados & Núm. de Atributos & Período & Referência \\
\hline \hline Despesas e Receita Municipais & 20 & 2002 até 2014 & [SEA18] \\
IDHM & 3 & 2010 & [dDHnB10] \\
Número de casos de Violência & 8 & 2001 até 2015 & [SEA18] \\
Profissionais da Saúde no COREN & 5 & 2001 até 2015 & [SEA18] \\
Taxas de Evasão escolar & 4 & 2007 até 2016 & [SEA18] \\
Densidade de Moradores por Dormitório & 3 & 2010 & [SEA18] \\
\hline
\end{tabular}

Tabela 3.2: Tabela das bases de dados socioeconômicos.

A descrição de cada base e a identificação das variáveis, serão apresentadas a seguir. A escolha das bases e atributos foi decidida em função do conhecido vínculo entre a doença e atributos. No entanto, é plenamente factível a inclusão de novas bases, atualização e substituição de dados, sem prejuízo da metodologia proposta neste trabalho.

São atributos comuns a todas as bases o código do município definido pelo IBGE e a localização dada pelas coordenadas (lat, long) do centro de cada município. Os atributos específicos serão listados após as bases.

Despesas e Receitas Municipais: Totais de despesas e receita em reais, atualizado em 2018. Atributos: Localidades; Desporto e Lazer; Educação; Receita Total; Legislativo; Judiciário; Comunicações; Defesa Nacional e Segurança Pública; Saúde; Atenção Básica; Assistência Hospitalar; Suporte Profilático; Vigilância Sanitária; Vigilância Epidemiológica; Alimentação e Nutrição; Saneamento Básico; Assistência Social; Previdência Social; Transporte; Cód. IBGE.

IDHM: Índice de Desenvolvimento Humano Municipal consolidado pelo censo de 2010. Atributos: Localidades; IDHM; Cód. IBGE.

Número de casos de Violência: Quantidade de ocorrências de violências notificadas por categoria, atualizado em 2017.

Atributos: Localidades; Homicídio Doloso; Latrocínio; Estupro; Total de Furtos; Total de Roubos; Total de Furtos e Roubos; Cód. IBGE. 
Profissionais da Saúde no COREN: Dados sobre a quantidade de profissionais registrados no COREN por categoria e médicos registrados no CRM, atualizado em 2017.

Atributos: Localidades; Técnico de Enfermagem; Enfermeiros; Médicos; Cód. IBGE.

Taxas de Evasão escolar: Dados sobre as taxas de evasão escolar, atualizado em 2018.

Atributos: Localidades; Taxa de Abandono no Ensino Fundamental; Taxa de Abandono no Ensino Médio; Cód. IBGE.

Densidade de Moradores por Dormitório: Densidade de moradores por dormitório em domicílios particulares permanentes, segundo censo 2010.

Atributos: Localidades; Mais de 2; Cód. IBGE.

Levando em consideração que a incidência de TB pode estar relacionada aos municípios próximos da faixa litorânea e naqueles que possuem ou estão próximos a presídios, foram criadas duas variáveis a partir dos dados dicotômicos originais:

- Distância do centro do município ao litoral ([Pau18]);

- Distância do centro do município aos presídios ([dAaP18]).

O cálculo da distância entre um município $i$ para uma localidade $j$ (litoral ou presídio), foi feita através da equação de Haversine:

$$
d_{i, j}=2 R \arcsin \left(\min \left\{1, \sqrt{\sin ^{2}\left(\frac{\phi_{i}-\phi_{j}}{2}\right)+\cos \left(\phi_{i}\right) \cos \left(\phi_{j}\right) \sin ^{2}\left(\frac{\lambda_{i}-\lambda_{j}}{2}\right)}\right\}\right) .
$$

De modo que, $(\phi, \lambda)$ são as latitudes e longitudes em radianos dos municípios, respectivamente, e $\mathrm{R}$ é o raio médio terrestre no valor de $6371 \mathrm{~km}$ ([Ven19] e [Wik18a]).

As siglas, variáveis e seus significados da base de dados socioeconômicas estão representados nas tabelas 3.3 e 3.4 . 


\begin{tabular}{|c|c|c|}
\hline Sigla & Variável & Significado \\
\hline$\overline{\mathrm{A} 1}$ & Taxa_de_Abandono_no_EF & $\begin{array}{l}\text { Porcentagem de alunos que abandonaram o ensino } \\
\text { fundamental antes da avaliação final ou que não } \\
\text { preencheram os requisitos mínimos em frequência } \\
\text { previstos em legislação, em relação ao total de alunos } \\
\text { matriculados no fim do ano letivo. }\end{array}$ \\
\hline $\mathrm{A} 2$ & Taxa_de_Abandono_no_EM & $\begin{array}{l}\text { Porcentagem de alunos que abandonaram o ensino } \\
\text { médio antes da avaliação final ou que não preencheram } \\
\text { os requisitos mínimos em frequência previstos em } \\
\text { legislação, em relação ao total de alunos matriculados } \\
\text { no fim do ano letivo. }\end{array}$ \\
\hline D1 & DR_Desp_e_Lazer & Taxa de despesas voltadas ao desporto e lazer. \\
\hline D2 & DR_Educacao & Taxa de despesas voltadas a educação. \\
\hline D3 & DR_Receita_Total & $\begin{array}{l}\text { Taxa de receitas orçamentárias recolhidas aos cofres } \\
\text { públicos. }\end{array}$ \\
\hline $\mathrm{D} 4$ & DR_Legislativo & $\begin{array}{l}\text { Taxa de despesas voltadas ao funcionamento dos } \\
\text { órgãos do Poder Legislativo. }\end{array}$ \\
\hline D5 & DR_Judiciario & $\begin{array}{l}\text { Taxa de despesas voltadas a defesa e acompanhamento } \\
\text { dos interesses da sociedade e do poder público. }\end{array}$ \\
\hline D6 & DR_Comunicacoes & Taxa de despesas voltada a comunicação. \\
\hline D7 & DR_Defesa_Nacional_e_SP & $\begin{array}{l}\text { Taxa de despesas voltadas para a garantia da segurança } \\
\text { pública e da prevenção da ordem. }\end{array}$ \\
\hline D8 & DR_Saude & Taxa de despesas na saúde municipal. \\
\hline D9 & DR_Atencao_Basica & $\begin{array}{l}\text { Taxa de despesas voltada ao atendimento das demandas } \\
\text { básicas da saúde. }\end{array}$ \\
\hline D10 & DR_Assistencia_Hospitalar & Taxa de despesas voltadas à cobertura de despesas com \\
\hline D11 & DR_Suporte_Profilatico & $\begin{array}{l}\text { internações hospitalares e tratamento ambulatorial. } \\
\text { Taxa de despesas voltadas para a produção, } \\
\text { distribuição e suprimento de drogas. }\end{array}$ \\
\hline D12 & DR_Vigilancia_S & Taxa de despesas voltadas à vigilância sanitária. \\
\hline D13 & DR_Vigilancia_E & $\begin{array}{l}\text { Taxa de despesas voltada ao combate e disseminação de } \\
\text { doenças transmissíveis. }\end{array}$ \\
\hline D14 & $\mathrm{DR}_{\text {_Alimentacao_N }}$ & $\begin{array}{l}\text { Taxa de despesas voltadas a diminuir ou eliminar } \\
\text { carências nutricionais. }\end{array}$ \\
\hline D15 & DR_Saneamento & $\begin{array}{l}\text { Taxa de despesas visando o fornecimento de água e } \\
\text { esgoto. }\end{array}$ \\
\hline D16 & DR_Assitencia_Social & Taxa de despesas voltadas para o bem-estar social. \\
\hline D17 & DR_Previdencia_Social & $\begin{array}{l}\text { Taxa de despesas governamentais de transferência de } \\
\text { renda aos cidadãos que sofrem de privação temporaria } \\
\text { da capacidade de prover seu próprio sustento. }\end{array}$ \\
\hline D18 & DR_Transporte & $\begin{array}{l}\text { Taxa de despesas voltadas a infra-estrutura e } \\
\text { o uso dos diversos meios de transporte. }\end{array}$ \\
\hline O1 & IDHM & Índice de Desenvolvimento Humano Municipal \\
\hline $\mathrm{O} 2$ & Dens_Dormitorio_mais_de_2 & $\begin{array}{l}\text { Densidade de moradores por dormitório maior } \\
\text { do que } 2 \text {. }\end{array}$ \\
\hline O3 & Dist_presidio & Distância até o município com o presídio mais próximo. \\
\hline $\mathrm{O} 4$ & Dist_litoral & Distância até o município litorâneo mais próximo. \\
\hline S1 & PS_Tecnico_de_Enfermagem & $\begin{array}{l}\text { Taxa de técnicos de enfermagem registrados } \\
\text { no COREN de São Paulo. }\end{array}$ \\
\hline
\end{tabular}

Tabela 3.3: Rótulos e significados dos atributos socioeconômicas (Parte 1). 


\begin{tabular}{r|l|l}
\hline Sigla & Variável & Significado \\
\hline \hline S2 & PS_Medicos & Taxa de médicos registrados no CRM de São Paulo. \\
\hline S3 & PS_Enfermeiros & Taxa de enfermeiros registrados no COREN de São Paulo. \\
\hline V1 & V_Homicidio_Doloso & Taxa de ocorrência de homicídio doloso. \\
\hline V2 & V_Roubo_Morte_Lat & Taxa de ocorrência de latrocínio. \\
\hline V3 & V_Estrupo & Taxa de ocorrência de estrupo. \\
\hline V4 & V_Furtos & Taxa de ocorrência de furtos. \\
\hline V5 & V_Roubos & Taxa de ocorrência de roubos. \\
\hline V6 & V_Furtos_e_Roubos & Taxa de ocorrência de furtos e roubos. \\
\hline
\end{tabular}

Tabela 3.4: Rótulos e significados dos atributos socioeconômicas (Parte 2).

\subsubsection{Tratamento dos dados socioeconômicos}

Comparações e análises foram feitas com base em dados normalizados pela população total residente em cada município. Os dados populacionais anuais foram extraídos das inferências do IBGE entre censos ([dGeEI19]). Para cada município $i$, a equação (3.6) foi usada para normalizar os atributos de despesas, receitas, violência e profissionais da saúde.

$$
(\text { Atributo_N })_{i}=\frac{(\text { Atributo_Anual })_{i}}{(\text { População Total residente })_{i}} \times 100 .
$$

Para a variável Densidade de Moradores por Dormitório foi adotada a normalização pela estimativa do total de domicílios particulares permanentes em cada município segundo a amostra do IBGE, visto que apresenta uma forte correlação linear significativa de 0,98 e p-valor $<0,001$, quando comparada com a normalização feita pela população total no município.

Como nem todas as bases de dados socioeconômicos coincidem no período disponível, foi selecionado o intervalo de tempo de 2007 até 2014, exceto para as variáveis decenais como IDHM, Densidade de Moradores por Dormitório cujos valores correspondem ao ano de 2010 e as distâncias aos presídios e ao litoral.

Uma análise dos dados faltantes indicou a necessidade de exclusão de variáveis cuja porcentagem de dados faltantes era maior ou igual a 50\%. A exclusão não levou em consideração o possível vínculo da variável com a doença, mas sim o impacto que a inclusão de valores inconsistentes poderia causar. Foram excluídas da análise despesas com Judiciário, Comunicações, Defesa Nacional e Segurança Pública, Assistência Hospitalar, Suporte Profilático, Vigilância Sanitária, Vigilância Epidemiológica, Alimentação e Nutrição, Saneamento, Previdência Social e variáveis de violência de Homicídio doloso e Latrocínio.

Após a aplicação do filtro de exclusão, foi feito um pré-processamento para completar os valores faltantes nas variáveis restantes. A Tabela 3.5 mostra as porcentagens de dados faltantes e outras estatísticas das variáveis socioeconômicas. 


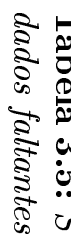

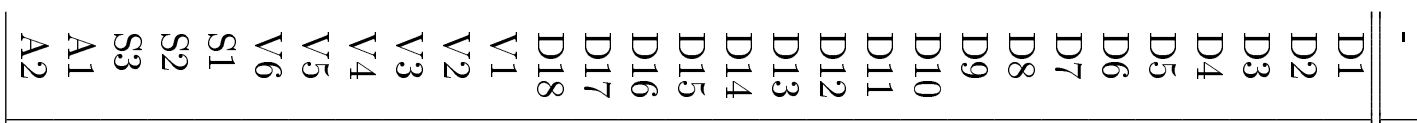

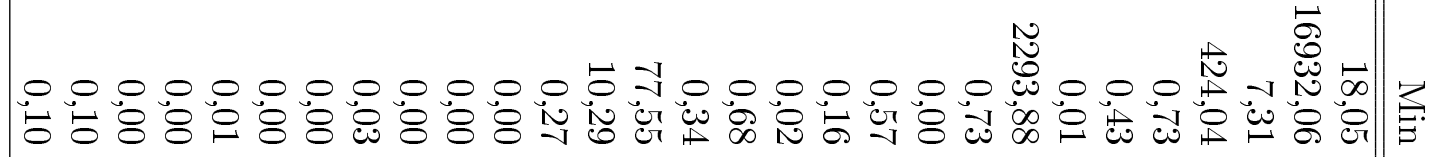

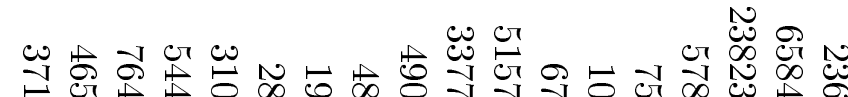

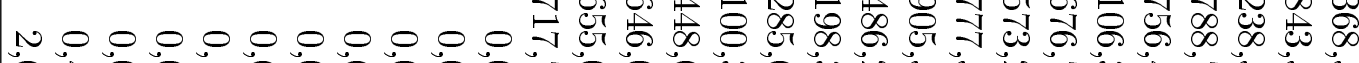

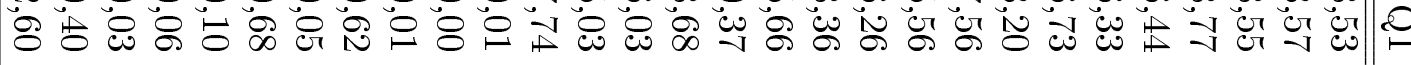

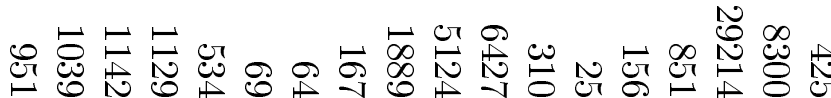

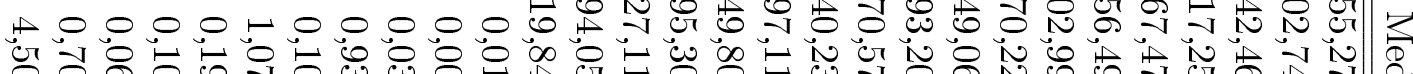

$\overrightarrow{\sigma_{0}}$

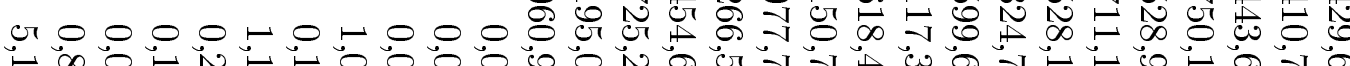

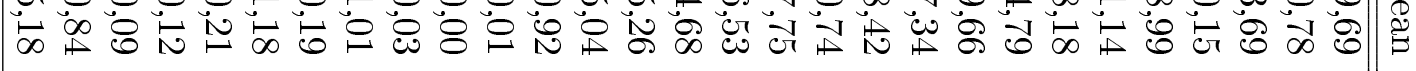

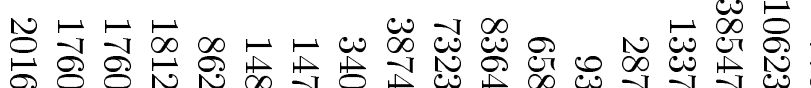

V-

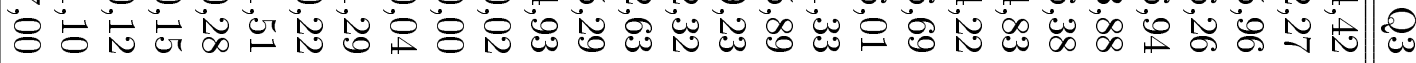

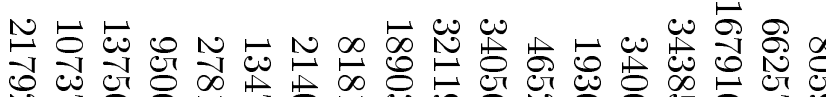
NoOO

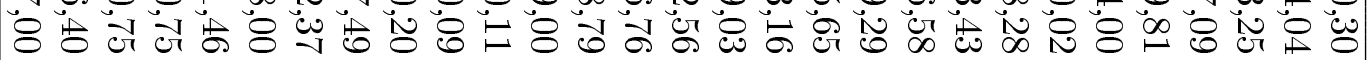

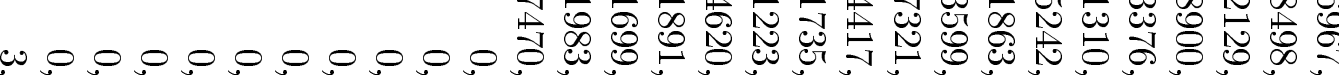

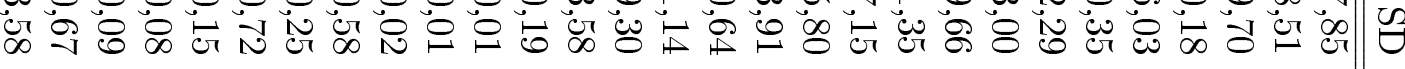

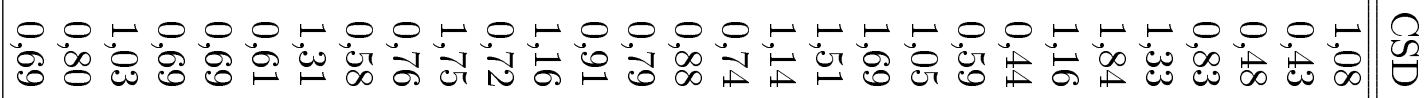
象

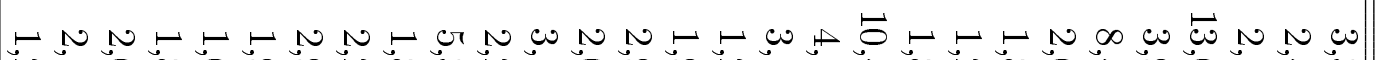

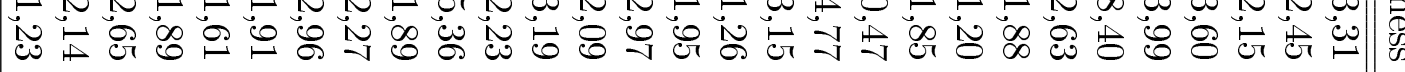

No o o o

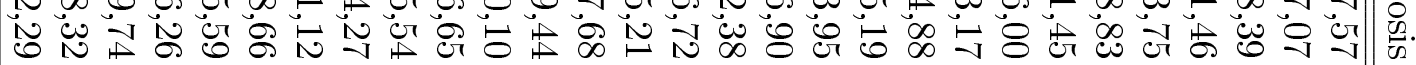

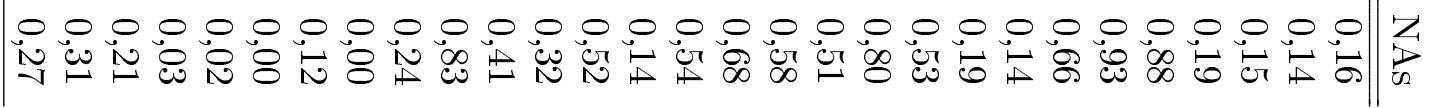


As principais estratégias pra completar os dados faltantes estão listadas a seguir e indicadas quais foram utilizadas neste trabalho ([Rub76] e [HK06]):

- Ignorar o dado: foi aplicado para eliminar os atributos com mais de $50 \%$ dos dados faltantes;

- Preencher o valor ausente manualmente e usar uma constante global no dado faltante: não foi aplicada, pois ignorar um município ou denotá-lo como NA impede a análise de dependência espacial que será utilizada posteriormente e, preencher manualmente não seria viável devido a quantidade massiva de dados, totalizando 5160 elementos (645 municípios e 8 anos) por variável;

- Uso da média do atributo no dado faltante: esse método foi descartado, devido ao valor alto do desvio padrão para algumas variáveis. A substituição pela média implicaria numa informação enviesada, principalmente devido à presença da capital com valores muito distintos quando comparado aos outros municípios;

- Uso da média do atributo para todas as amostras pertencentes à mesma classe que o objeto especificado: esse método também foi descartado, pois não temos as classes dos municípios. Uma possível estratégia seria realizar um agrupamento em cada variável e depois completar os dados utilizando os grupos como classes, porém nem todos os municípios possuem dados completos em todas as variáveis;

- Uso do valor mais provável: essa estratégia foi utilizada com o auxílio do algoritmo EM - Expectation Maximization de Dempster et al. ([DLR77]) com Bootstrap e um polinômio de grau 2 para a série temporal em cada município, junto com a abordagem de imputação múltipla desenvolvida por Rubin ([Rub87]). A função polinomial de grau 2 foi incluída para suavizar os valores imputados em cada município. Ao invés de utilizar várias bases de dados separadamente, utilizou-se a média das 100 imputações gerando uma única base de dados completa.

Testes estatísticos foram aplicados para verificar quais variáveis são MAR ou MCAR e identificar a qualidade dos dados. Na Tabela 3.6 a diagonal superior dessa matriz indica se há ou não relações entre as variáveis, utilizando uma tabela qui-quadrado, afim de identificar se os dados faltantes de uma variável está relacionada com outra. Os valores que não estão em vermelho indicam relação significativa com o nível de significância de 5\%, apontando as variáveis que são MAR. 


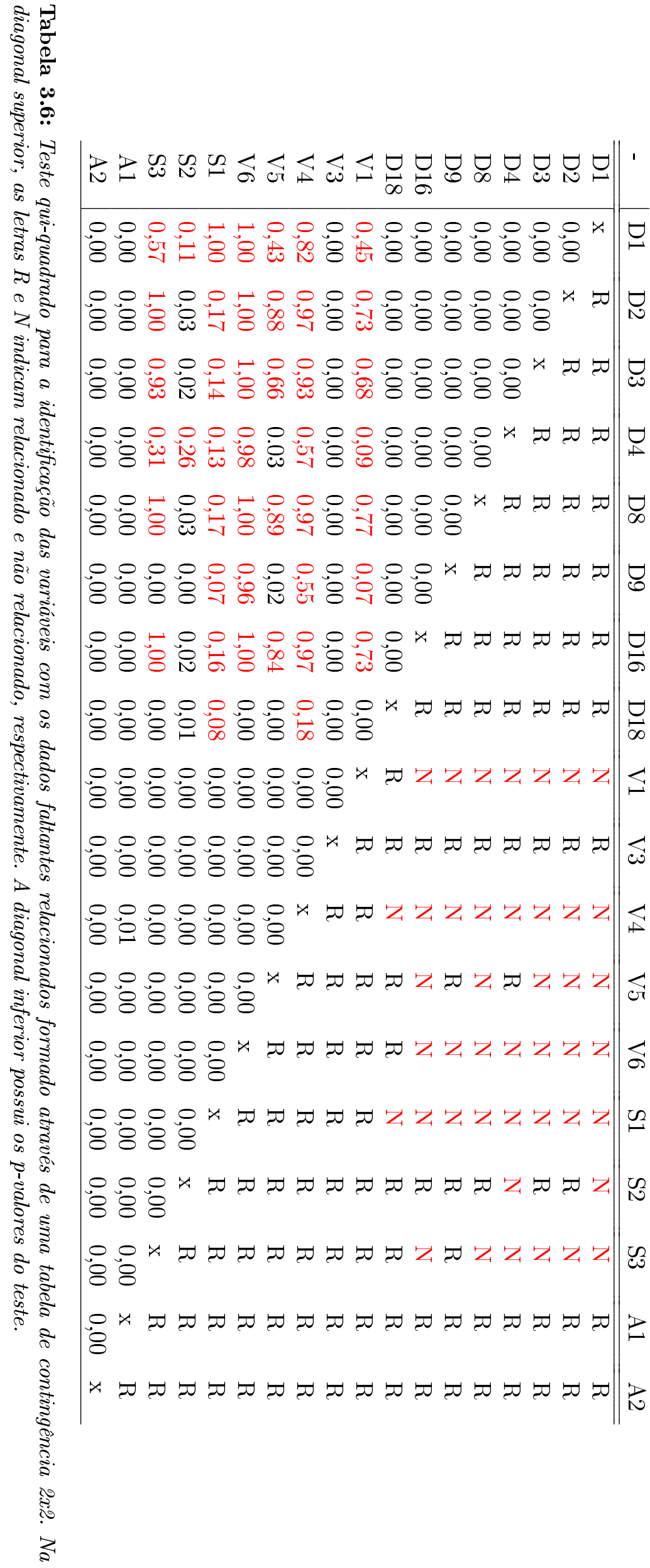


O pacote que disponibiliza esse algoritmo é o Amelia do R Studio proposto por James Honaker, Gary King e Matthew Blackwell em 2011 ([HKB11]). Entretanto, ao invés de utilizar todo o conjunto de dados no algoritmo, foi utilizado apenas duas variáveis, sendo o tempo uma delas. Isso foi feito, pois ao utilizar todo o conjunto de dados, fontes de imputação negativa surgem, fugindo do modelo real. Existe um método chamado Predictive Mean Matching (PMM) que contorna esse tipo de problema ([Rub87], p. 168), porém ele falha ao utilizar a base de dados completa devido a existência de municípios que não possuem nenhum valor em todas as variáveis (Figura 3.3) e fica limitado aos menores e maiores valores dos dados observados. A Figura 3.3 identifica a variável que apresenta o maior proporção de dados faltantes V1, que representa a taxa de violência por homicídio doloso.
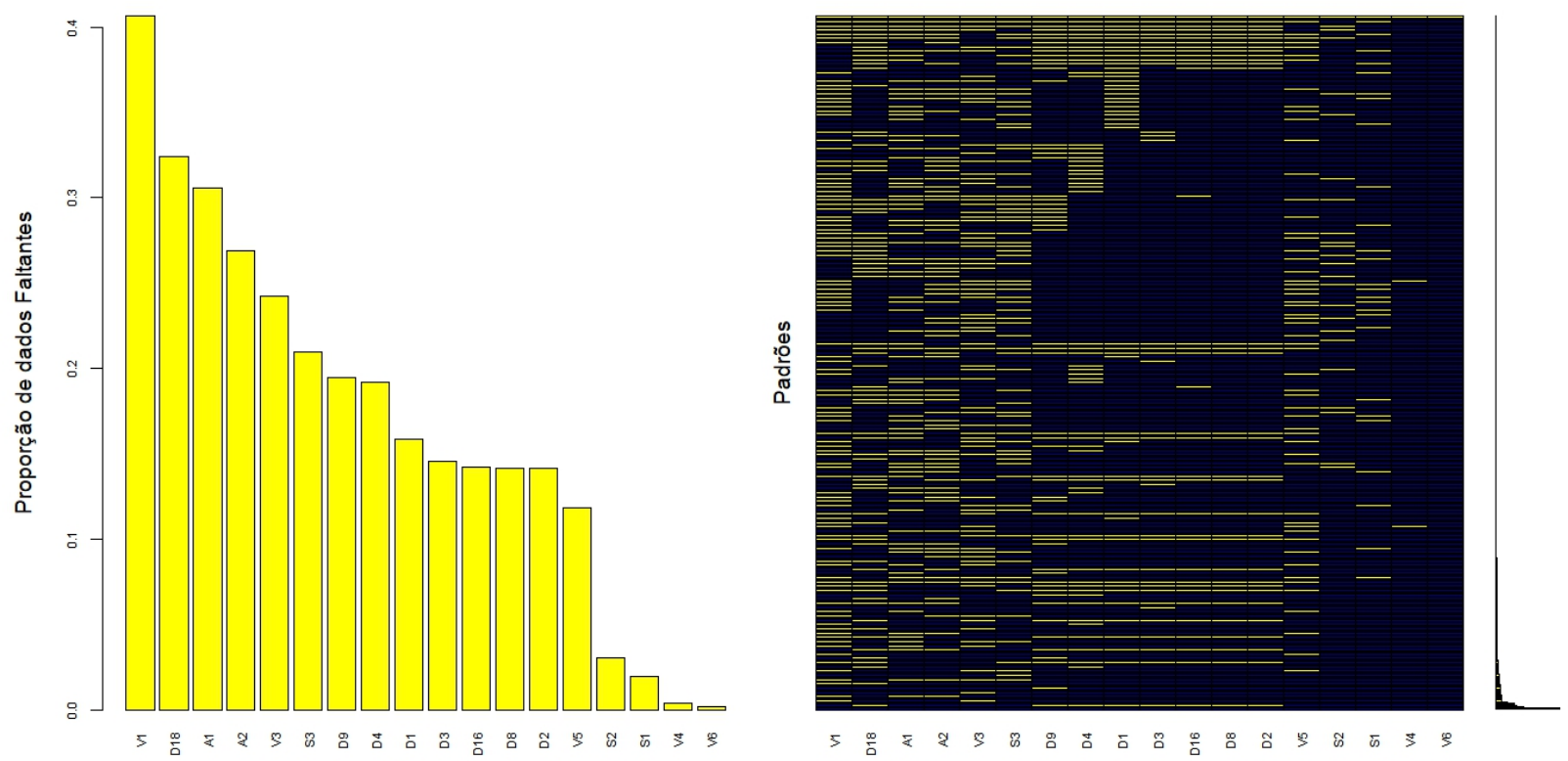

Figura 3.3: Proporção de dados faltantes (lado esquerdo) e padrões dos dado faltantes em amarelo e não faltantes em azul (lado direito).

As distribuições dos dados observados e as médias dos dados faltantes inseridos estão representados nas Figuras 3.4 e 3.5. Conforme James et al. ([HKB11], p. 28), apesar das distribuições dos dados inseridos e observados não estarem parecidos, isso não significa que o modelo de inclusão esteja errado, mas mostra onde está ocorrendo a maior quantidade de inclusões, como ocorre com as variáveis DR_Atencao_Basica, DR_Transporte, DR_Desp_e_Lazer, PS_Enfermeiros, Taxa_de_Abandono_no_EF, Taxa_de_Abandono_no_EM e V_Homicidio_Doloso.

As Figuras 3.6 e 3.7 mostram as distribuições dos dados observados e completos. No caso das variáveis DR_Desp_e_Lazer, DR_Transporte e PS_Tecnico_de_Enfermagem os dados observados possuem um erro padrão baixo, fazendo com que os valores imputados se acumulem próximo da média dos dados. Já para as variáveis que apresentam a forma bimodal é justificada devido a inclusão da ordem do tempo. 

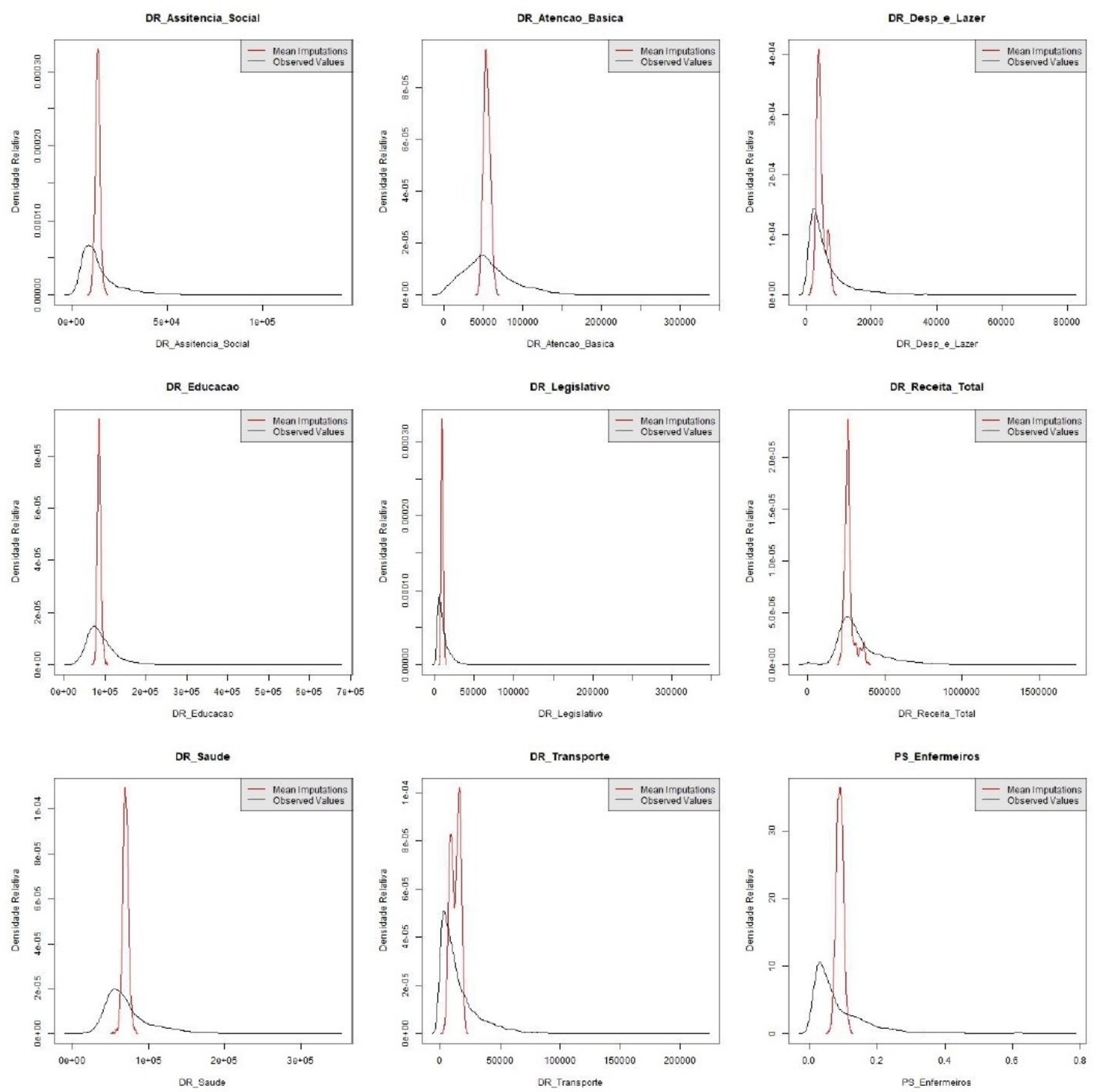

Figura 3.4: Parte 1: Distribuições dos dados observados (preto) e das médias dados faltantes para $m=100$ (vermelho) das variáveis selecionadas. 

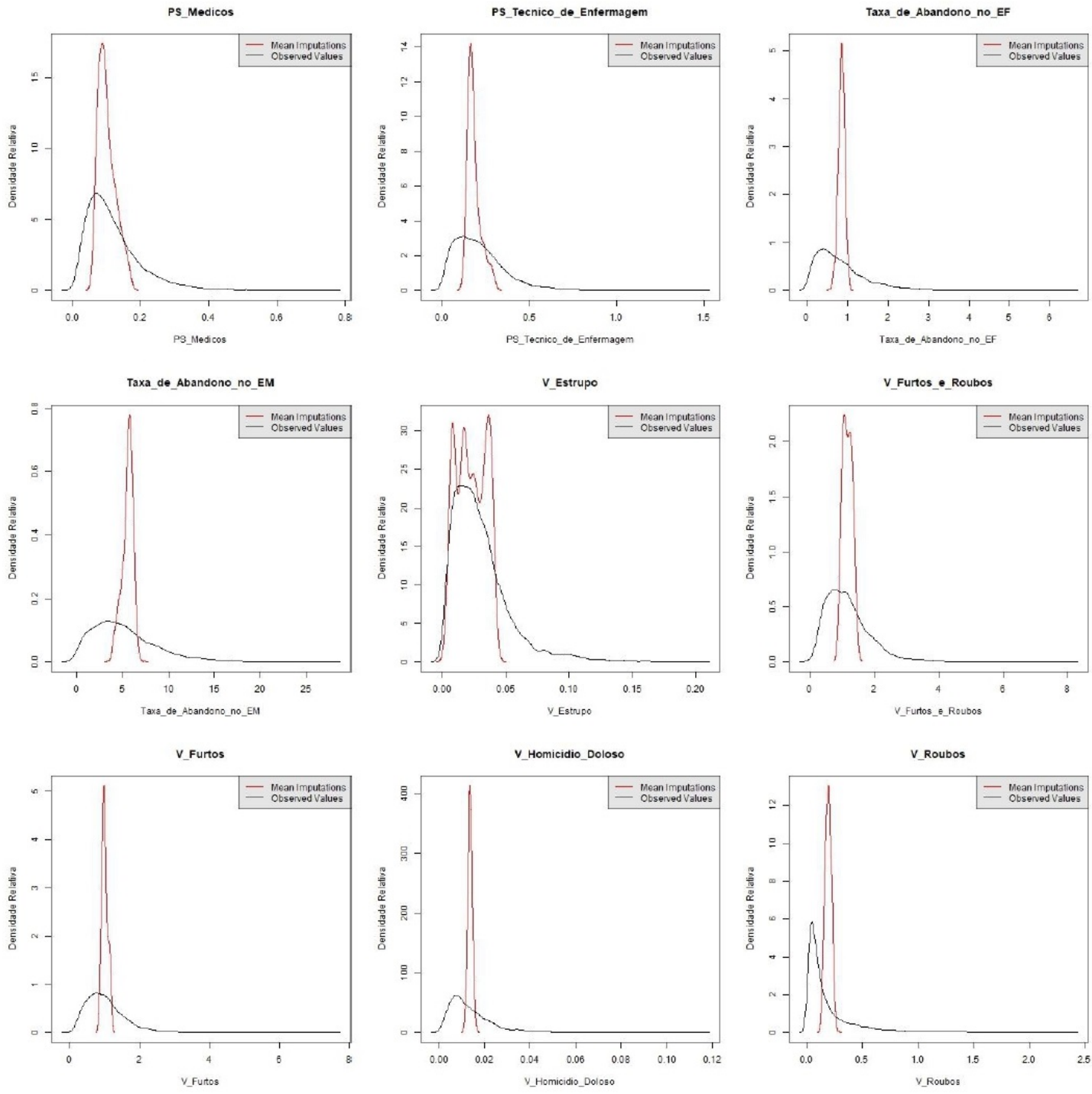

Mean Imputations

Observed Values

Figura 3.5: Parte 2: Distribuições dos dados observados (preto) e das médias dados faltantes para $m=100$ (vermelho) das variáveis selecionadas. 

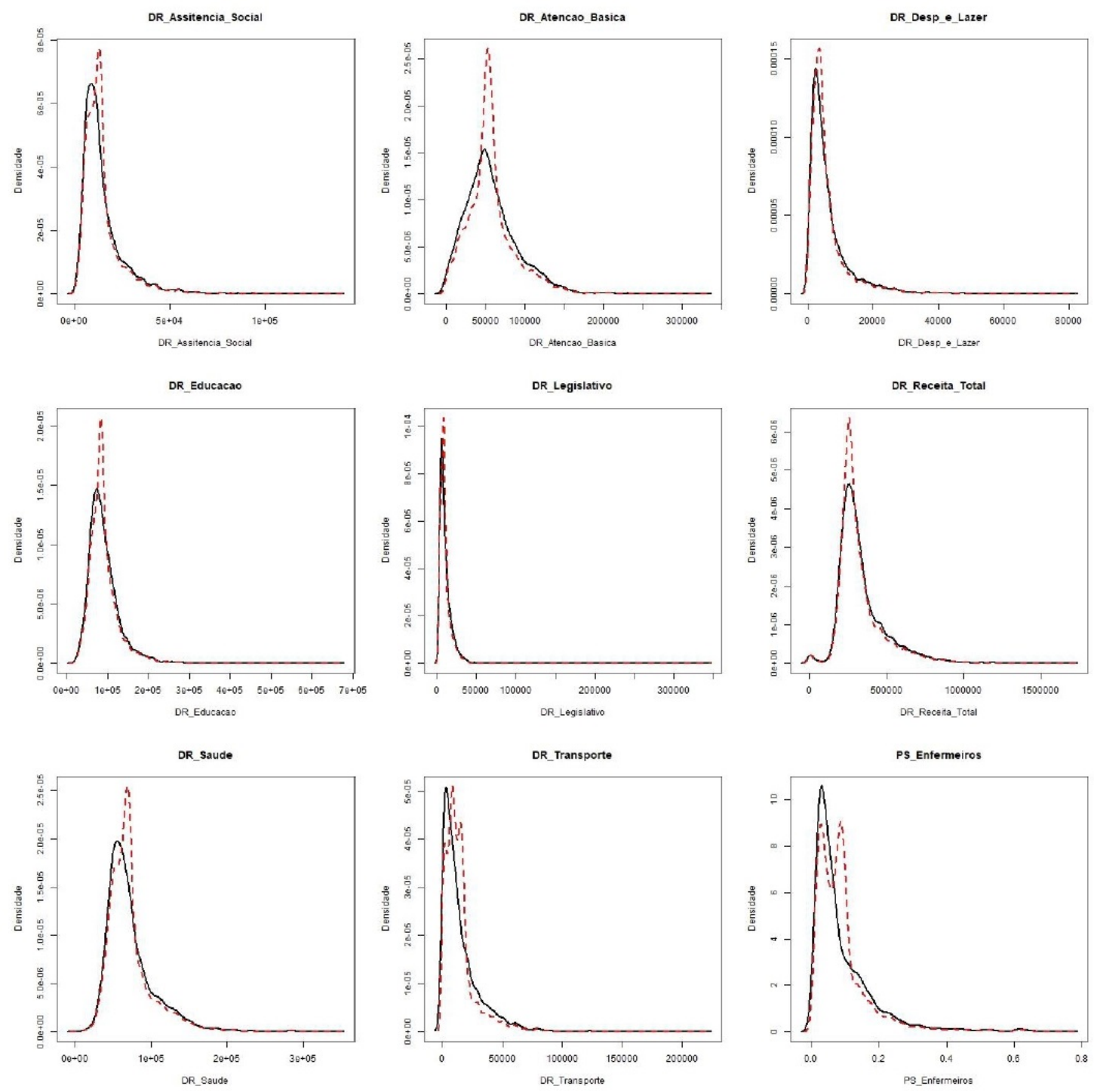

Figura 3.6: Parte 1: Distribuição dos dados observados (preto) e dos dados completos (vermelho). 

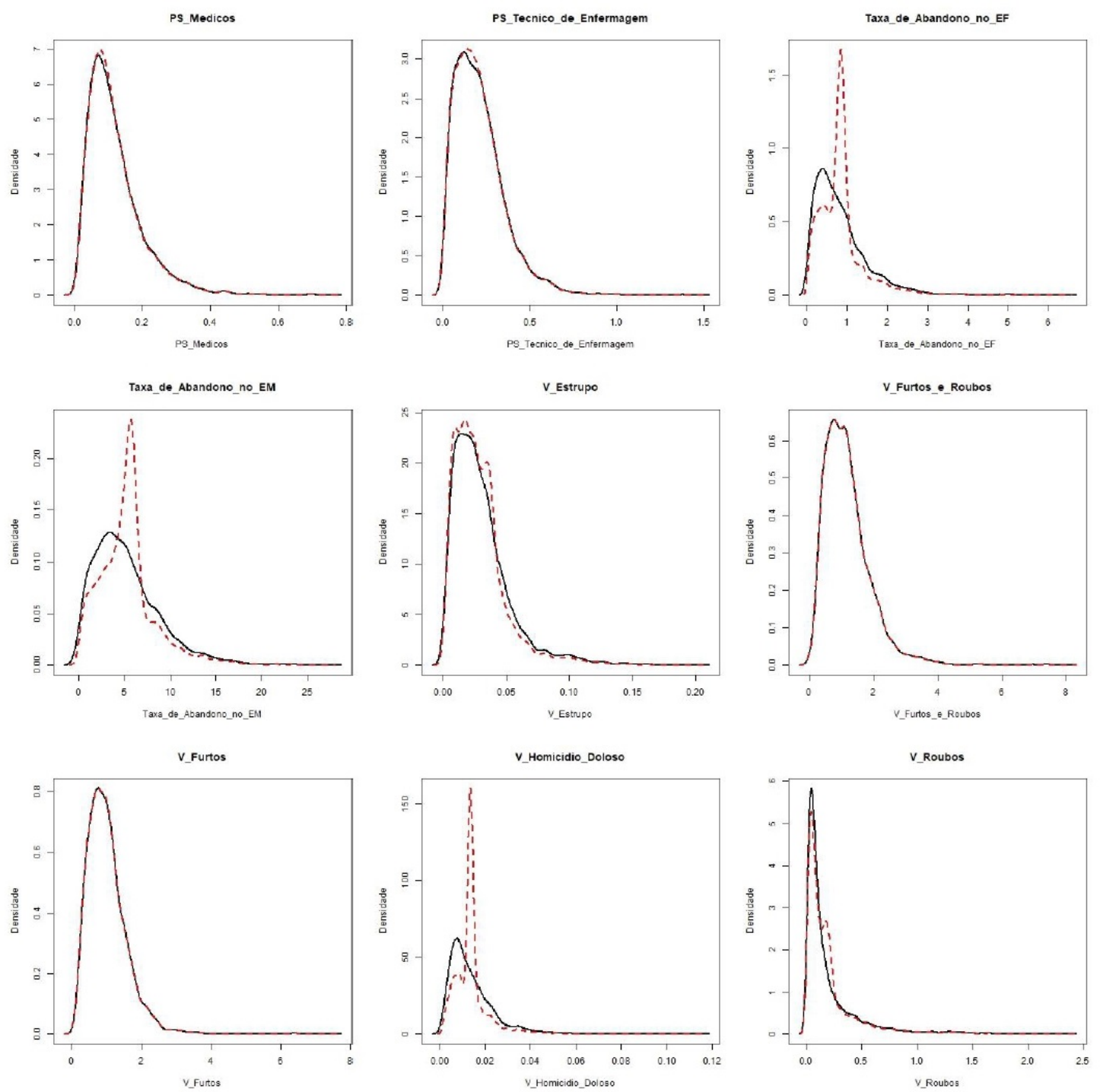

Figura 3.7: Parte 2: Distribuição dos dados observados (preto) e dos dados completos (vermelho). 
Com os dados completos, optou-se por analisar a média temporal de cada município, transformando os dados espaço-temporais para dados espaciais. Isso permitiu concatenar variáveis do IDHM e densidade de moradores por dormitório. Essa metodologia acarreta os seguintes pontos negativos:

- Inclusão do viés da média em todos os municípios, pois no período selecionado o desvio padrão é não nulo, pelo menos em um município para algumas variáveis;

- Impossibilidade de analisar os efeitos temporais de cada variável.

Apesar desses pontos, o método permite a inclusão de outras variáveis que não dependem do tempo e que podem auxiliar na análise do problema como, por exemplo, alguns índices que são atualizados em cada censo.

A análise sobre a multicolinearidade entre as variáveis foi feita por meio da correlação linear de Spearman e o método de agrupamento hierárquico AGNES, com a utilização do método Average e a métrica de proximidade definida pela equação (2.14). Essa etapa é optativa, porém auxilia na identificação e exclusão de variáveis dependentes identificadas pelo algoritmo de associação, reduzindo a dimensionalidade do problema e, consequentemente, o espaço de busca e o tempo de processamento.

A Figura 3.8 mostra o dendograma gerado o qual permite identificar facilmente as variáveis com altas correlações absolutas (acima de 0,5) e excluir as variáveis redundantes, tais como, taxa de abandono no ensino médio, taxa de furto, taxa de furto e roubo e receita municipal.

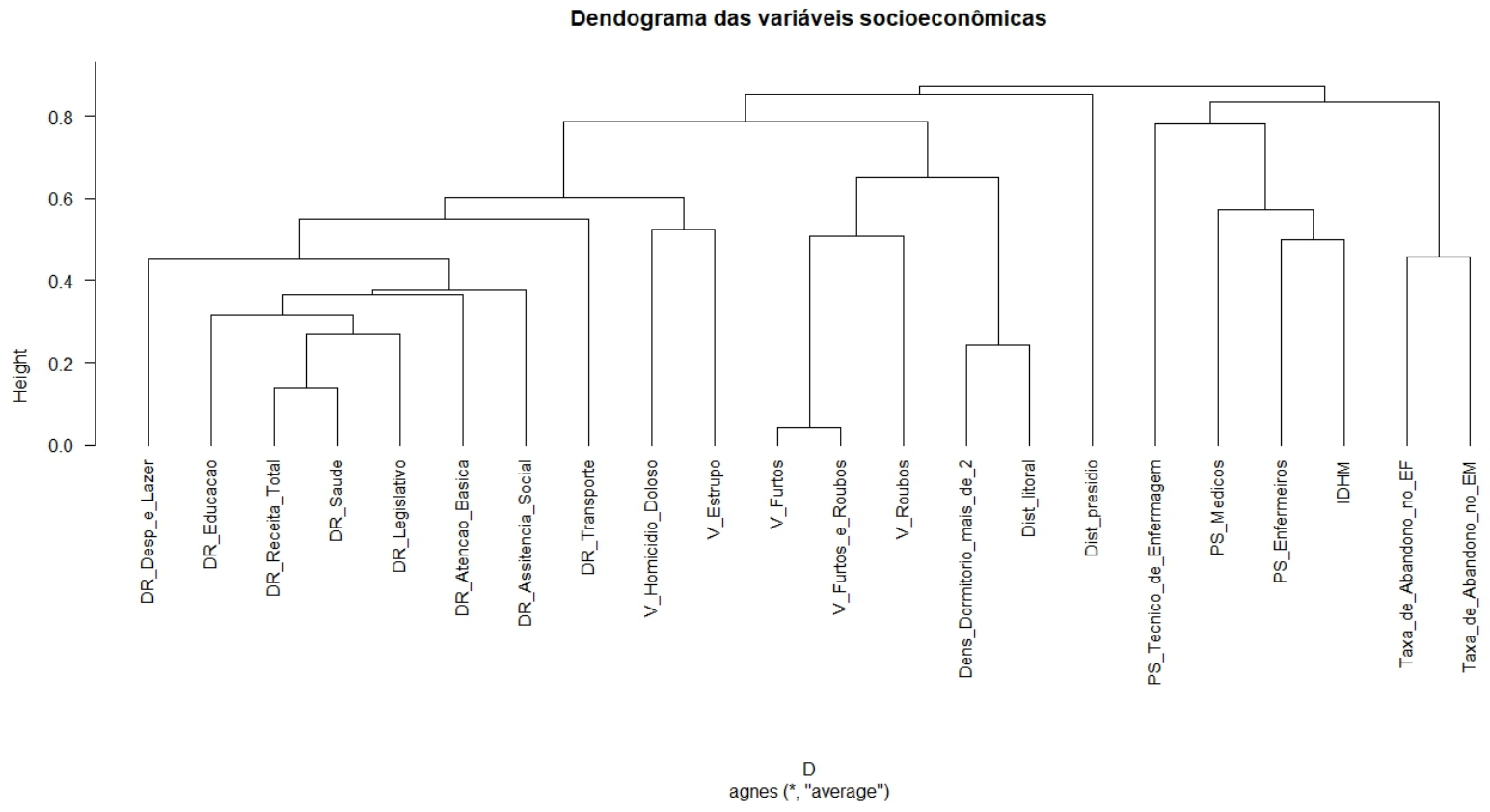

Figura 3.8: Dendograma do agrupamento realizado através do algoritmo hierárquico AGNES para investigar as variáveis mais correlacionadas.

Em resumo, as bases que serão mineradas são: Socio, TS, MI e FX que representam, respectivamente, as variáveis socioeconômicas, série temporal anual discreta da incidência de tuberculose, 
média da taxa de incidência e a faixa etária normalizada mais atingida. A Tabela 3.7 ilustra a base de dados Socio com os atributos quantitativos contínuos e qualitativos nominais.

\begin{tabular}{c|l|l|l}
\hline Siglas & Atributo & Tipo & Base de Dados \\
\hline \hline ID_Mun & Cód. IBGE & Nominal & - \\
Nom_Mun & Localidade & Nominal & - \\
D1 & DR_Desp_e_Lazer & Contínua & Despesas e Receita Municipais \\
D2 & DR_Educacao & Contínua & Despesas e Receita Municipais \\
D4 & DR_Legislativo & Contínua & Despesas e Receita Municipais \\
D8 & DR_Saude & Contínua & Despesas e Receita Municipais \\
D9 & DR_Atencao_Basica & Contínua & Despesas e Receita Municipais \\
D16 & DR_Assitencia_Social & Contínua & Despesas e Receita Municipais \\
D18 & DR_Transporte & Contínua & Despesas e Receita Municipais \\
V1 & V_Homicidio_Doloso & Contínua & Número de casos de Violência \\
V3 & V_Estupro & Contínua & Número de casos de Violência \\
V5 & V_Roubos & Contínua & Número de casos de Violência \\
S1 & PS_Tecnico_de_Enfermagem & Contínua & Profissionais da Saúde no COREN \\
S2 & PS_Enfermeiros & Contínua & Profissionais da Saúde no COREN \\
S3 & PS_Medicos & Contínua & Profissionais da Saúde no COREN \\
A1 & Taxa_de_Abandono_no_EF & Contínua & Taxas de Evasão escolar \\
O1 & IDHM & Contínua & IDHM \\
O2 & Dens_Dormitorio_mais_de_2 & Contínua & Densidade de Moradores por Dormitório \\
O3 & Dist_Presidio & Contínua & Secretaria de Administração Penitenciária \\
O4 & Dist_Litoral & Contínua & Litoral Paulista \\
\hline
\end{tabular}

Tabela 3.7: Caracterização da base de dados Socio com os atributos socioeconômicos após o tratamento das variáveis. 


\subsection{Métodos}

A metodologia proposta neste trabalho para a extração dos padrões consiste em pré-processamento, análise descritiva, aplicação dos algoritmos de Mineração de Dados e pós-processamento como forma de avaliar os padrões obtidos, utilizando os dados Socio, FX, TS e MI, conforme ilustra a Figura 3.9 .

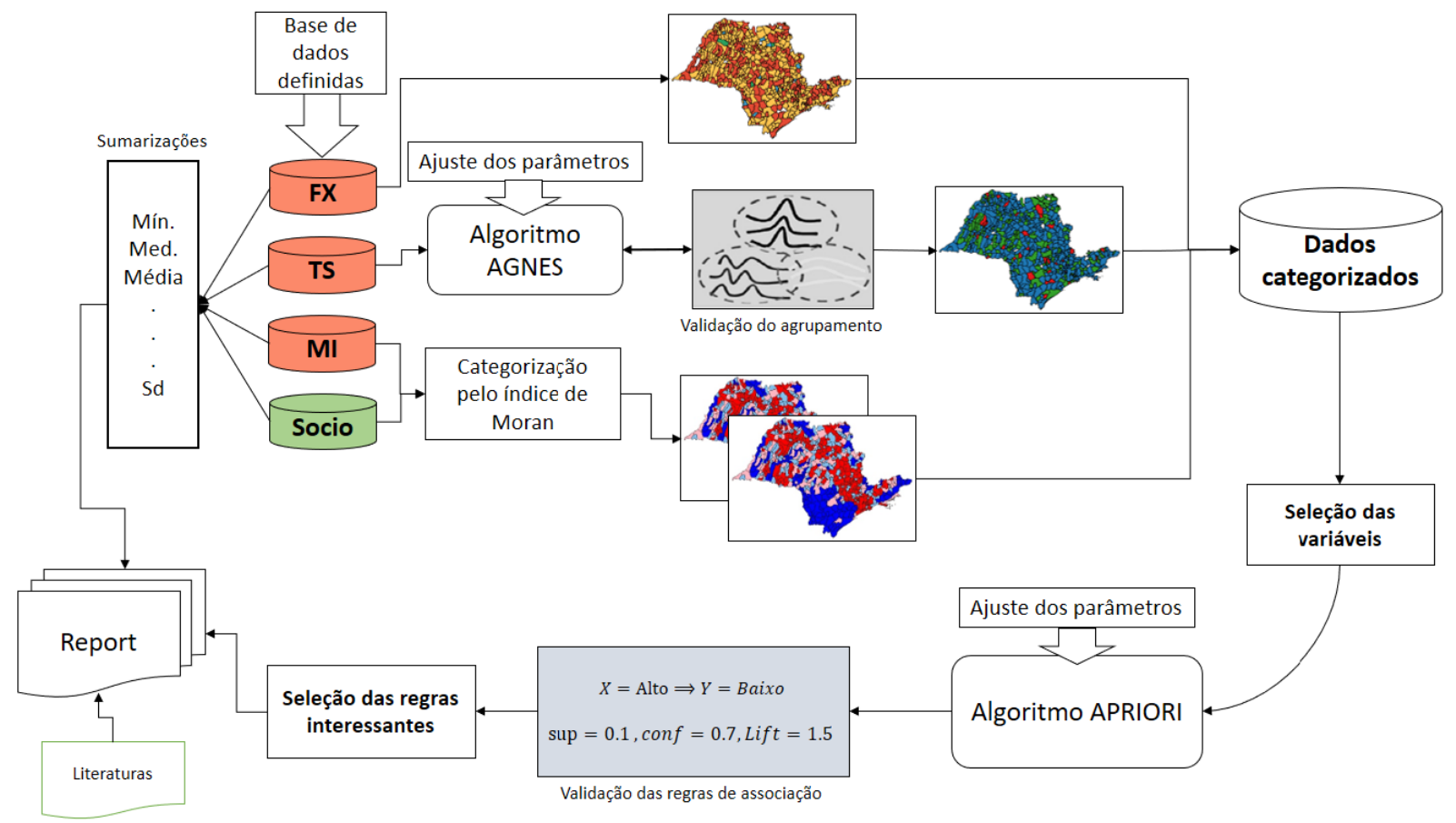

Figura 3.9: Metodologia adotada para a extração dos padrões nos dados de tuberculose.

As regras de associação entre as categorias das variáveis de cada base de dados foi extraída com o uso do algoritmo APRIORI, descrito no capítulo 2 na seção 2.6. A categorização das variáveis numéricas obtidas através dos dados Socio e MI foi feita com o uso do diagrama de Moran. O algoritmo AGNES foi aplicado nas séries temporais da incidência de tuberculose para diagnosticar o comportamento crescente e decrescente de cada série e relacionar com as localidades de maior risco através dos dados MI. As sumarizações foram necessárias para obter as estatísticas dos conjuntos de dados e averiguar os padrões obtidos.

\subsubsection{Pré-processamento}

Cada atributo da base de dados Socio foi padronizado pela equação Z-score, definida conforme a equação (3.7). Essa padronização permite que a média seja nula e o desvio padrão unitário, reduzindo os efeitos dos outliers presentes nas variáveis. Por ser usualmente aplicado em dados epidemiológicos ([BPGR08], p. 275), a padronização dos dados MI foi feita através da equação (3.8). Ela foi utilizada, principalmente pelos dados MI apresentarem uma distribuição fortemente assimétrica, devido a presença de valores discrepantes. Para os dados TS a normalização min-max (equação (3.9)) foi aplicada, pois ela permite que os valores permaneçam no mesmo intervalo $[0 ; 1]$.

$$
\mathbf{x}_{z-\text { score }}=\frac{(\mathbf{x}-\overline{\mathbf{x}})}{s d[\mathbf{x}]}
$$




$$
\begin{gathered}
\mathbf{x}_{l o g}=\log _{10}(\mathbf{x}+1) \\
\mathbf{x}_{\text {min-max }}=\frac{\mathbf{x}-\min (\mathbf{x})}{\max (\mathbf{x})-\min (\mathbf{x})}
\end{gathered}
$$

O algoritmo APRIORI necessita de um conjunto de dados cujos atributos são variáveis qualitativas ou categóricas, discretizamos alguns atributos. Há diversos métodos de discretização ([HK06], [TSK06] e [LL14]). Os mais usuais são:

- Equal width binning ou interval: Divide o tamanho do atributo (diferença do maior e menor valor) em $\mathrm{k}$ intervalos de mesmo comprimento;

- Equal frequency binning ou frequency: Divide o tamanho do atributo em $\mathrm{k}$ intervalos, de modo que cada intervalo contenha aproximadamente a mesma frequência ou número de dados;

- Intuitive partitioning ou fixed: Utiliza do conhecimento do especialista para discretizar os dados. Geralmente, os valores menores do que o $1^{\mathrm{o}}$ quantil são ditos baixos, valores entre o $1^{\mathrm{O}}$ e o $3^{\mathrm{O}}$ quantil são ditos médios e valores maiores que o $3^{\mathrm{O}}$ quantil são altos;

- Clustering: Utiliza algoritmos de agrupamento para agrupar os dados, gerando uma discretização em termos dos grupos obtidos.

Os métodos de discretização indicados acima particionam os dados num certo valor numérico podendo não estar de acordo com o que os analistas sugerem. Por exemplo, ao particionar a variável da média de incidência de tuberculose nos últimos 3 anos analisados, utilizando $k=3$, obtivemos diferentes partições numéricas (Tabela 3.8). Portanto, categorizar os municípios tanto pelo seu valor numérico quanto pelo valor dos seus vizinhos, permite identificar padrões espaciais e relacionar essas categorizações de cada região entre as variáveis. Por esse motivo, optou-se por utilizar a metodologia do diagrama de Moran para categorizar as variáveis dos dados Socio e MI. O pré-processamento realizado em cada base de dados está representado na Tabela 3.9.

\begin{tabular}{c|cccc}
\hline Partições/Método & Interval & Frequency & Fixed & Clustering \\
\hline \hline 1 & {$[0 ; 290)$} & {$[0 ; 8,53)$} & {$[0 ; 5,85)$} & {$[0 ; 105)$} \\
2 & {$[290 ; 581)$} & {$[8,53 ; 19,2)$} & {$[5,85 ; 23,8)$} & {$[105 ; 404)$} \\
3 & {$[581 ; 871]$} & {$[19,2 ; 871]$} & {$[23,8 ; 871]$} & {$[404 ; 871]$} \\
\hline
\end{tabular}

Tabela 3.8: Discretizações obtidas por diferentes métodos aplicados à variável da média da incidência nos últimos 3 anos (2015, 2016 e 2017).

O IDHM possui categorias pré estabelecidas que informam o grau de desenvolvimento social nos municípios do Brasil ([dDHnB10]). Como o coeficiente de variação (CSD) da variável IDHM para todos os municípios do estado de São Paulo é menor do que 0,1, ou seja, cerca de 580 municípios possuem IDHM maior do que 0,7 , isso faz com que $89 \%$ dos dados sejam categorizados como Bom ou Ótimo. Portanto, optou-se por discretizar essa variável também com o uso da metodologia adotada pelo diagrama de Moran. 


\begin{tabular}{|c|c|c|c|c|}
\cline { 2 - 5 } \multicolumn{1}{c|}{} & \multicolumn{4}{c|}{ Pré-processamento } \\
\hline Dados & Limpeza & Integração & Transformação & Redução \\
\hline Socio & $\begin{array}{c}\text { Tratamento dos } \\
\text { dados faltantes }\end{array}$ & $\begin{array}{c}\text { Inclusão de dados de outras } \\
\text { fontes IDHM, densidade de } \\
\text { moradores por dormitório, } \\
\text { distância do presídio e } \\
\text { do litoral. }\end{array}$ & $\begin{array}{c}\text { Z-score } \\
\text { e } \\
\text { Categorização } \\
\text { por Moran }\end{array}$ & $\begin{array}{c}\text { Eliminação das variáveis } \\
\text { com mais de 50\% dos } \\
\text { dados faltantes } \\
\text { e correlacionadas. }\end{array}$ \\
\hline FI & - & - & $\begin{array}{c}\text { Logarítmica e } \\
\text { Categorização } \\
\text { por Moran }\end{array}$ & - \\
\hline TS & - & - & - & - \\
\hline
\end{tabular}

Tabela 3.9: Pré-processamento realizado em cada base de dados. 


\subsubsection{Análise Descritiva dos Dados}

Algumas estatísticas foram extraídas do conjunto de dados TS (Tabela 3.10) e Socio (Tabela 3.11) para auxiliar na análise dos dados. A segunda tabela possui as estatísticas após o tratamento dos dados, conforme descrito em Materiais (seção 3.1). Além das estatísticas extraídas no conjunto de dados em cada variável, também foram analisados as estatísticas por município, referente ao conjunto de dados da doença (TS, FX e MI) e socioeconômicos (Socio). Por ser uma base de dados muito grande, quando concatenadas, essas estatísticas não serão apresentadas em formas de tabelas e suas análises serão descritas nos Resultados.

O número de municípios categorizados segundo a metodologia do diagrama de Moran está representado na Tabela 3.12, na qual a coluna Não Signif. indica o número de municípios que foram categorizados como não significativos, sendo a Ilha Bela o único município que não possui vizinhos de primeira ordem presente nesta categoria.

\begin{tabular}{l|rrrrrrrrrrr}
\hline- & Min & Q1 & Med & Mean & Q3 & Max & SD & CSD & Skewness & Kurtosis & Notif \\
\hline \hline 2001 & 0,00 & 0,00 & 17,86 & 24,14 & 33,65 & 1208,16 & 53,72 & 2,23 & 16,88 & 363,64 & 438 \\
2002 & 0,00 & 0,00 & 18,65 & 24,63 & 33,22 & 1226,26 & 54,14 & 2,20 & 17,22 & 374,06 & 454 \\
2003 & 0,00 & 0,00 & 18,87 & 25,02 & 33,48 & 792,35 & 41,43 & 1,66 & 10,52 & 181,58 & 453 \\
2004 & 0,00 & 0,00 & 15,22 & 24,46 & 30,73 & 743,17 & 45,85 & 1,87 & 8,14 & 104,17 & 436 \\
2005 & 0,00 & 0,00 & 14,41 & 47,62 & 27,80 & 15431,35 & 608,74 & 12,78 & 25,03 & 629,37 & 424 \\
2006 & 0,00 & 0,00 & 13,67 & 22,59 & 27,59 & 591,23 & 43,17 & 1,91 & 6,62 & 63,56 & 426 \\
2007 & 0,00 & 0,00 & 14,03 & 23,31 & 27,96 & 507,10 & 41,65 & 1,79 & 5,79 & 49,60 & 434 \\
2008 & 0,00 & 0,00 & 15,38 & 23,91 & 28,53 & 364,88 & 40,62 & 1,70 & 4,74 & 29,98 & 442 \\
2009 & 0,00 & 0,00 & 13,62 & 23,84 & 28,33 & 766,45 & 47,79 & 2,00 & 7,93 & 98,27 & 445 \\
2010 & 0,00 & 0,00 & 15,48 & 26,70 & 31,49 & 546,76 & 49,67 & 1,86 & 5,54 & 41,50 & 447 \\
2011 & 0,00 & 0,00 & 14,55 & 25,48 & 28,70 & 746,27 & 54,46 & 2,14 & 7,50 & 76,86 & 451 \\
2012 & 0,00 & 0,00 & 13,88 & 25,48 & 26,77 & 590,70 & 54,44 & 2,14 & 5,62 & 38,90 & 445 \\
2013 & 0,00 & 0,00 & 13,32 & 25,66 & 28,09 & 721,86 & 54,98 & 2,14 & 6,47 & 57,55 & 438 \\
2014 & 0,00 & 0,00 & 13,52 & 27,16 & 27,35 & 756,10 & 62,71 & 2,31 & 6,76 & 59,38 & 441 \\
2015 & 0,00 & 0,00 & 12,39 & 26,30 & 25,78 & 704,82 & 63,29 & 2,41 & 6,43 & 50,20 & 439 \\
2016 & 0,00 & 0,00 & 12,75 & 28,47 & 27,36 & 898,92 & 71,22 & 2,50 & 7,08 & 65,11 & 444 \\
2017 & 0,00 & 0,00 & 13,26 & 32,19 & 27,32 & 1175,79 & 87,98 & 2,73 & 7,54 & 73,85 & 442 \\
\hline
\end{tabular}

Tabela 3.10: Sumarizações das variáveis temporal da incidência de tuberculose nos municípios do estado de São Paulo. Como não tem dados faltantes, a coluna Notif. indica o total de municipios que notificaram tuberculose no respectivo ano. 


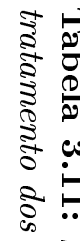

कृ

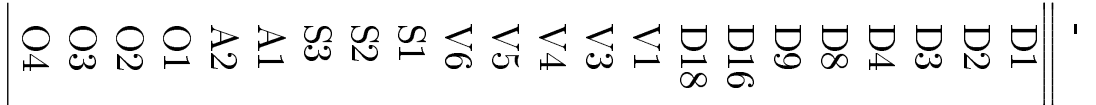

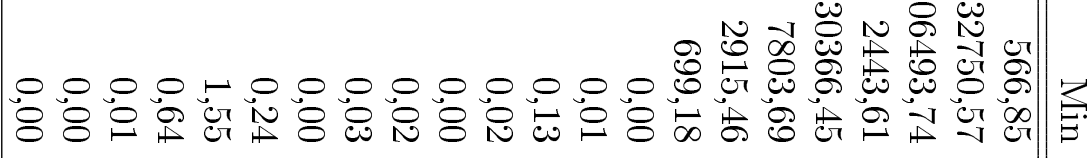

ถุ

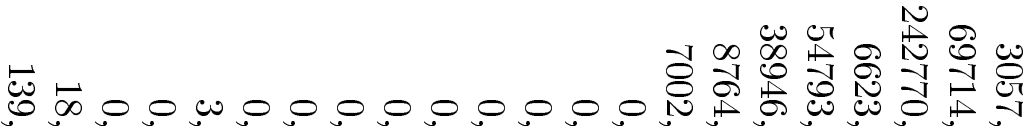

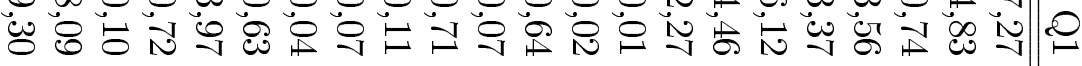

ن क 木

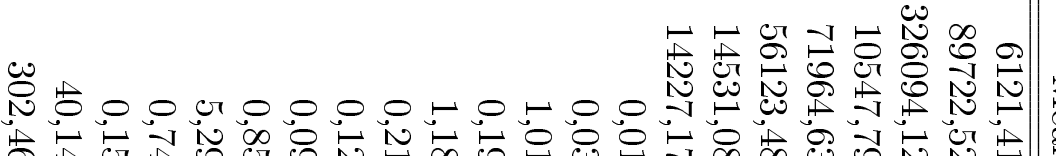

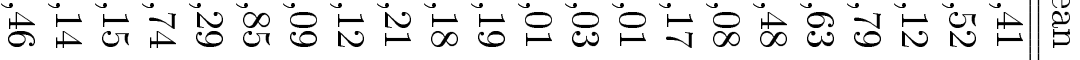

花 ن

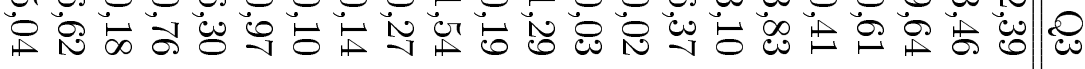
S

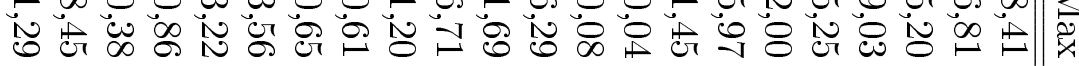

Tَ 넌 0000000000500000000000 光

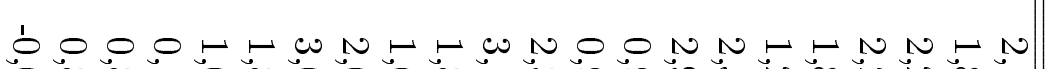

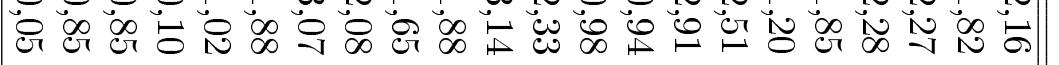

$\frac{\delta^{2}}{8}$

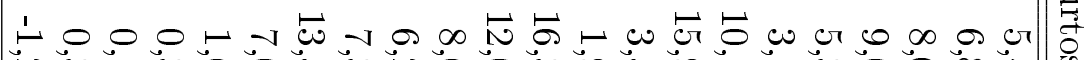
N 


\begin{tabular}{l|ccccc}
\hline Variável & Alto-Alto & Alto-Baixo & Baixo-Alto & Baixo-Baixo & Não Signif. \\
\hline \hline D1 & 83 & 132 & 117 & 312 & 1 \\
D2 & 126 & 143 & 119 & 256 & 1 \\
D4 & 95 & 132 & 101 & 316 & 1 \\
D8 & 125 & 107 & 109 & 303 & 1 \\
D9 & 124 & 147 & 107 & 266 & 1 \\
D16 & 118 & 98 & 118 & 310 & 1 \\
D18 & 111 & 109 & 117 & 307 & 1 \\
V1 & 121 & 203 & 111 & 209 & 1 \\
V3 & 170 & 113 & 100 & 261 & 1 \\
V5 & 131 & 28 & 51 & 434 & 1 \\
S1 & 208 & 65 & 115 & 256 & 1 \\
S2 & 154 & 89 & 151 & 250 & 1 \\
S3 & 112 & 103 & 251 & 178 & 1 \\
A1 & 133 & 110 & 132 & 269 & 1 \\
O1 & 229 & 83 & 166 & 166 & 1 \\
O2 & 211 & 64 & 63 & 306 & 1 \\
O3 & 222 & 51 & 15 & 356 & 1 \\
O4 & 331 & 5 & 9 & 299 & 1 \\
MI & 62 & 65 & 117 & 400 & 1 \\
\hline
\end{tabular}

Tabela 3.12: Variáveis com o número de municípios em cada categoria obtida pela metodologia do diagrama de Moran. 


\subsubsection{Agrupamento das Séries Temporais da incidência de TB}

Existem diversos métodos capazes de agrupar um conjunto de séries temporais e podem ser encontrados nos trabalhos de Saeed Aghabozorgi et al. ([ASW15]) e Pjotr Roelofsen ([Roe18]). Alguns softwares como o que foi introduzido por Pablo Monteiro et al. ([MV14]) na linguagem R permitem o tratamento e análise de séries temporais, além de agrupamento e predição.

O agrupamento das séries temporais dos dados TS foi realizado utilizando a taxonomia whole time-series clustering que procura agrupar uma série temporal completa através de uma métrica de proximidade, conforme Saeed et al. ([ASW15], p. 18). O algoritmo aplicado para gerar o agrupamento foi o AGNES.

Várias métricas de correlação foram testadas para agrupar as séries temporais da incidência de tuberculose, utilizando correlação de Pearson, Spearman e a correlação proposta por Ahlame D. Chouakria et al. (eq. (2.16)). A métrica que teve a melhor qualidade no agrupamento é dada pela equação (3.10):

$$
d(\mathbf{x}, \mathbf{y})=\frac{1-r_{s}(\mathbf{x}, \mathbf{y})}{2} .
$$

Na equação (3.10), a função $(\mathbf{x}, \mathbf{y}) \mapsto r_{s}(\mathbf{x}, \mathbf{y})$ é a correlação amostral de Spearman entre dois vetores. Como o interesse é relacionar apenas o comportamento da série com as variáveis em algumas regiões, esse método foi o mais adequado ao problema proposto.

Os municípios que não notificaram tuberculose no período foram categorizados e separados antes do processo de agrupamento. Isso foi necessários, pois ao aplicar a correlação de Spearman o desvio padrão é nulo e a métrica não fica bem definida.

O principal desafio no agrupamento é encontrar o número ideal de grupos. A variação do número de grupos (cortes no dendograma) e variação dos parâmetros do algoritmo é possível identificar o melhor resultado com o uso dos índices de avaliação.

Aplicando o algoritmo AGNES com o parâmetro de união de grupos Average e a métrica definida pela equação (3.10) obtemos o dendograma representado na Figura 3.10. O CPCC desse dedograma foi de $0.4498(95 \%, \mathrm{IC}=[0,4463 ; 0,4533])$, indicando uma correlação linear moderada entre a matriz de dissimilaridade e o dendograma obtido pelo algoritmo.

A Figura 3.11 mostra a variação do índice de Silhouette em relação ao número de grupos ou cortes do dendograma. É possível observar que o melhor índice está entre 200 a 300 grupos, mostrando a heterogeneidade das séries nos municípios. Optou-se por ficar com 2 grupos que definem o melhor índice para a menor quantidade de grupos. O índice de Silhouette médio foi 0,15 e o $S C=0,17$, sendo que a perda da qualidade de agrupamento foi de 19,04\% em relação ao melhor agrupamento encontrado.

A Figura 3.12 representa o gráfico de barras do índice de Silhouette aplicado aos dados TS para 


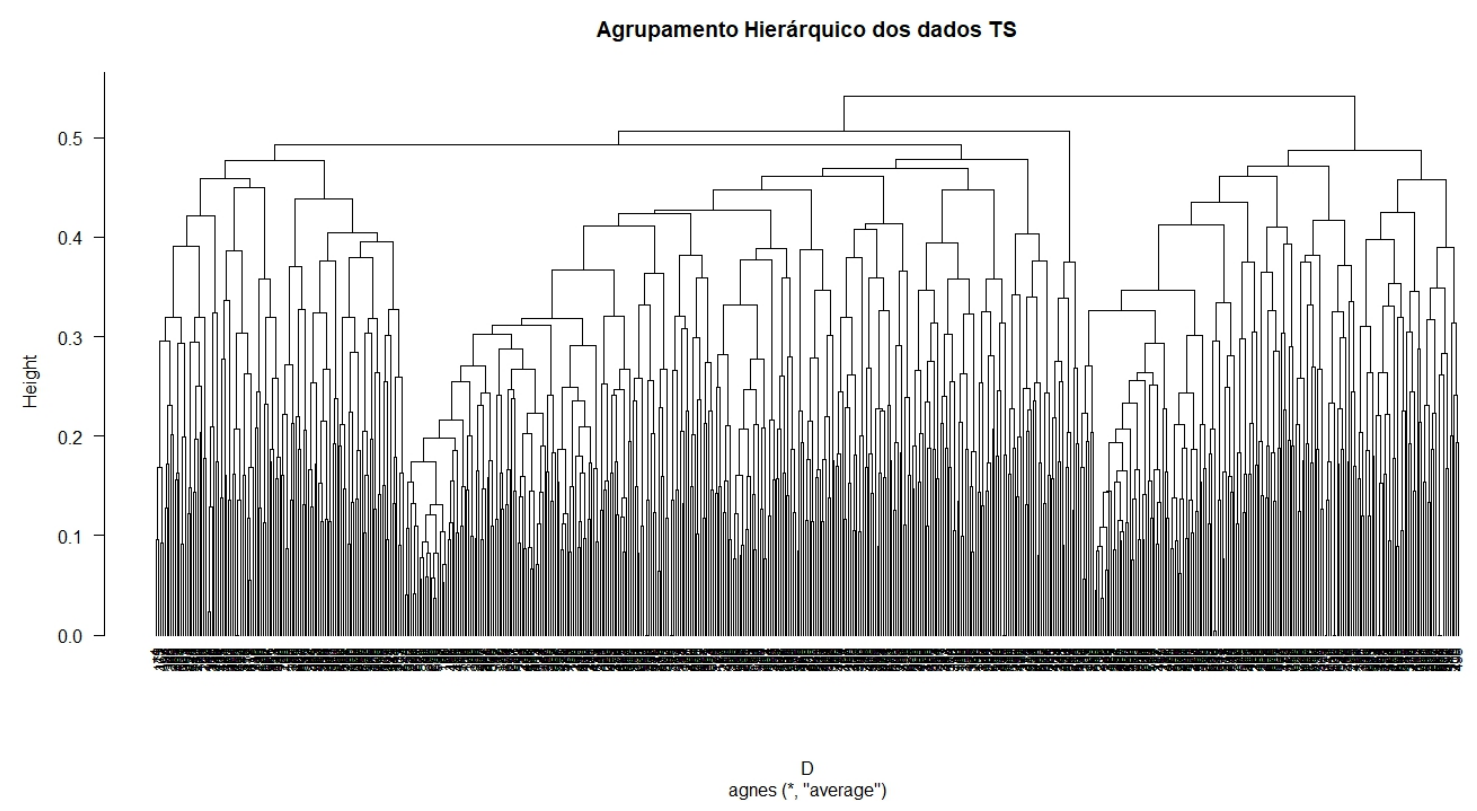

Figura 3.10: Dendograma dos dados TS gerado pelo algoritmo AGNES com o parâmetro Average e métrica de dissimilaridade dada por 3.10 .

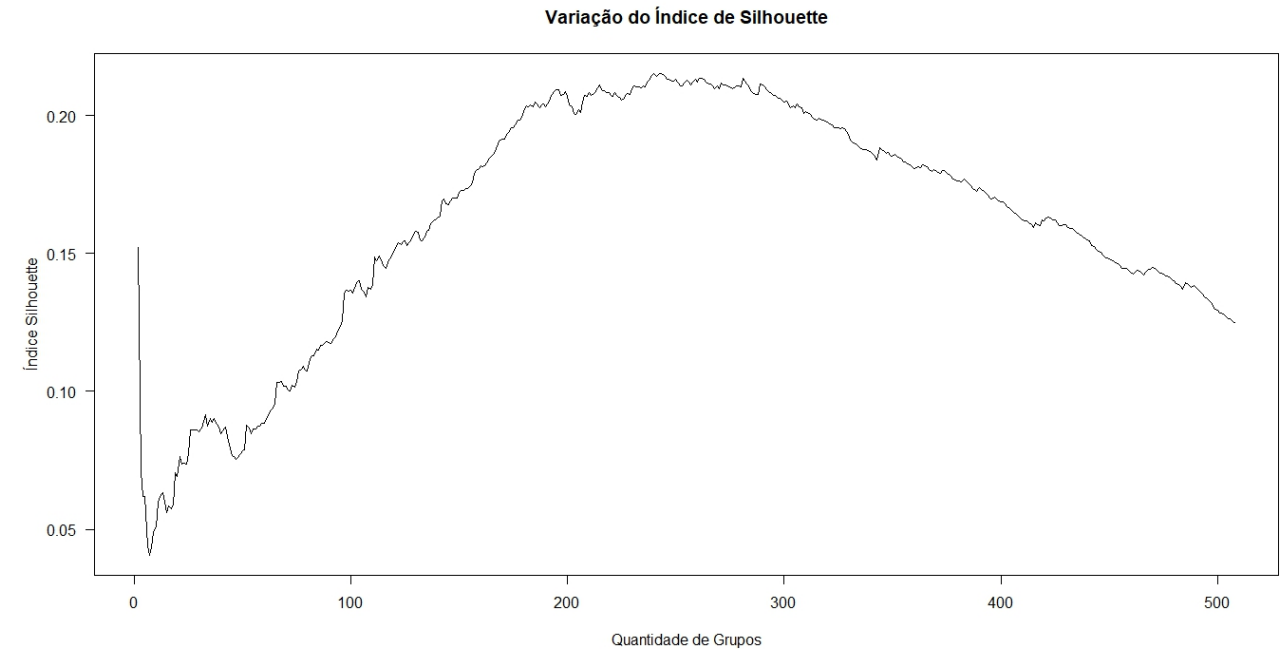

Figura 3.11: Variação do indice de Silhouette em relação ao número de grupos, ou cortes no dendograma gerado pelo algoritmo AGNES.

2 grupos. O primeiro grupo possui 450 objetos, de modo que o Silhouette médio desse grupo é 0,14, sendo possível identificar que alguns municípios possuem valores negativos desse índice. Já o outro grupo possuí um índice médio maior $\bar{s}_{2}=0,17$. 
Silhouette plot of ( $x=$ cutree(hc.D, $k=2$ ), dist = D)

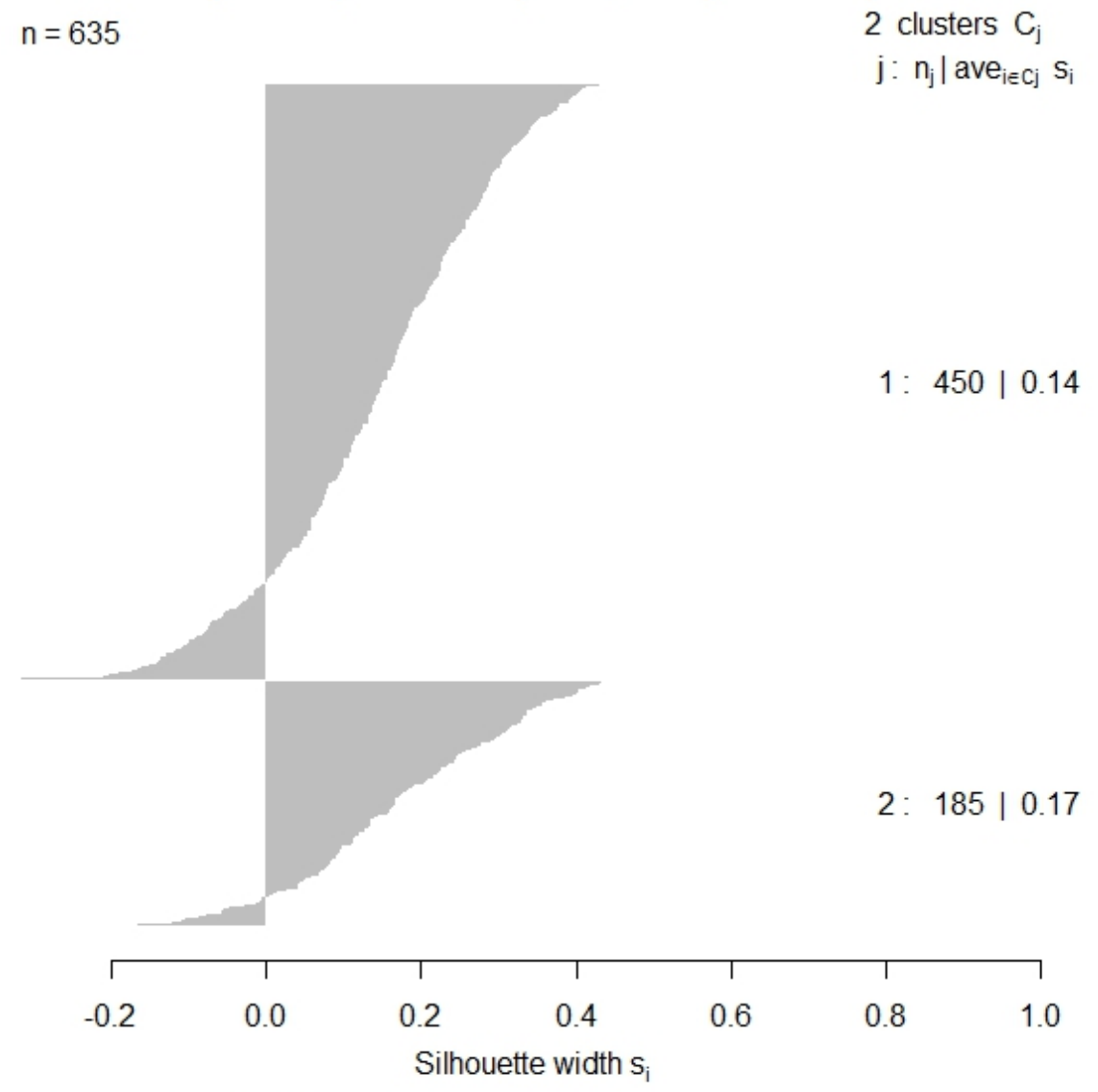

Average silhouette width : 0.15

Figura 3.12: Resultado do indice de Silhouette para um agrupamento da série temporal na base TS, através do algoritmo hierárquico AGNES, com o parâmetro Average e a métrica definida pela equação (3.10). 


\subsubsection{Aplicação da Análise de Associação}

Após tratar as bases de dados (diagrama de Moran) e definir as classes para as séries temporais (agrupamento), foi aplicado o algoritmo APRIORI, que busca associações e extrai padrões do tipo $A \Longrightarrow B$, entre as bases dados ([HTF01], [Alp04], [HK06], [TSK06], [LL14] e [dSPB16]). A aplicação e análise dos resultados do algoritmo foram feitas em duas etapas:

1. Extração dos itens frequentes: obtenção e análise dos itens frequentes obtidos do conjunto de dados;

2. Extração das regras: geração e análise das regras fortes e aplicação dos índices de avaliação descritos no capítulo precedente.

Diferentes valores para o parâmetro suporte foram testados e o número de regras e tempo de execução analisados. A Figura 3.13 mostra a variação do total de regras encontradas e tempo de execução do algoritmo para cada suporte analisado. Para um suporte de 30\%, somente 16 regras foram encontradas e para um suporte de $3 \%, 367242$ regras.

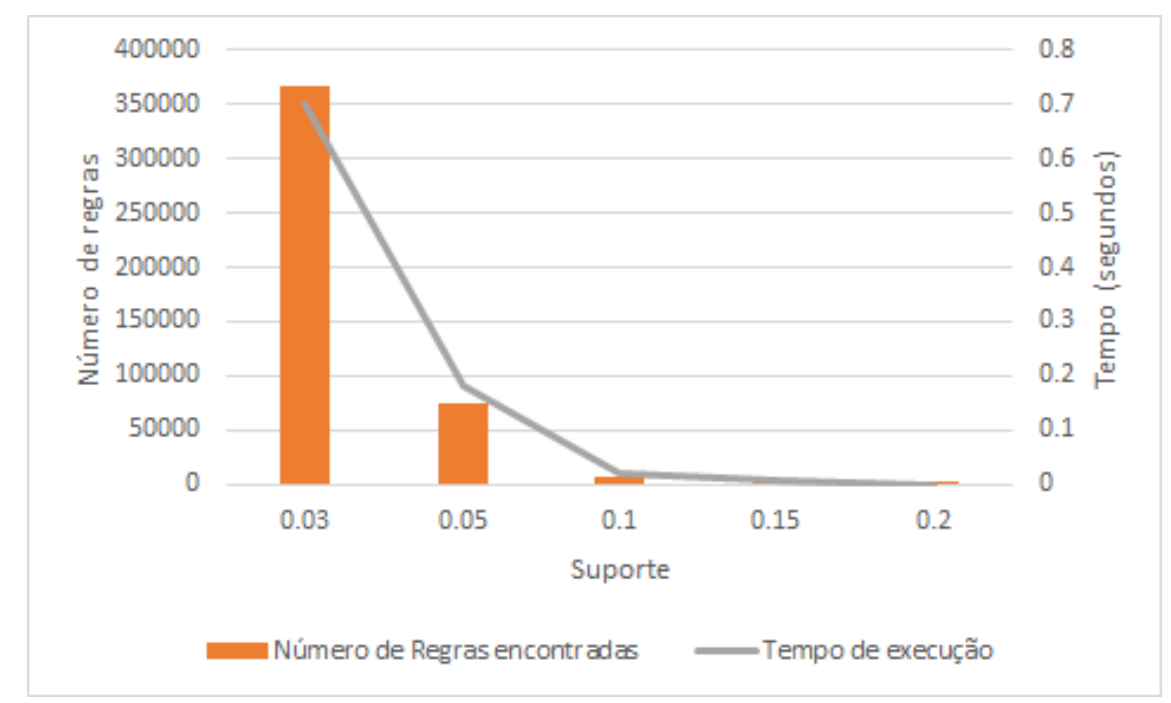

Figura 3.13: Variação do número de regras e tempo de execução para os suportes: 3\%, 5\%, 10\%, $15 \%$ e $20 \%$.

A partir da variação dos parâmetros suporte e confiança, cada regra extraída foi pós-processada através dos índices de avaliação, para valores do suporte maior que 3\%. Alguns consequentes e antecedentes foram fixados, com o propósito de verificar a relação entre determinadas categorias e as variáveis disponíveis. 


\section{Capítulo 4}

\section{Resultados e Discussão}

\subsection{Análise Descritiva dos Dados}

Com base nos dados brutos de tuberculose, de 2001 até 2017, foram notificados cerca de 324218 casos de tuberculose no estado de São Paulo, sendo 277046 casos novos. O ano de 2017 registrou o maior número de casos novos, cerca de 18031. O município de São Paulo registrou os maiores números de casos novos com 6666 em 2002 e 6665 em 2017.

O município de Borá apresentou um crescimento atípico que merece a revisão dos dados cadastrados, pois registrou a taxa acima de 1500 casos por 100 mil habitantes.

A taxa de incidência média no estado de São Paulo foi de 39,59 casos por 100 mil habitantes $(95 \%, \mathrm{IC}=[38,50 ; 40,68])$. A maior taxa ocorreu em $2002 \mathrm{com} 44,92$ casos por 100 mil hab. e a menor em 2016 com 37,38 casos por 100 mil habitantes.

As taxas de incidência dependem das variáveis Casos Novos, Não Sabe e Pós-óbito por TB, conforme o regulamento descrito no DATASUS ([DAT18]), a Figura 4.1 mostra o comportamento dessas variáveis ao longo do tempo.

A categoria Casos Novos varia em torno da média de 16297 casos $(95 \%, \mathrm{IC}=[15965 ; 16629])$. A categoria Não Sabe apresentou uma queda após 2002 quando atingiu 90 casos, seu maior valor. Finalmente, a categoria Pós-óbito, que apresentou um crescimento expressivo após 2011, com mais de 150 casos registrados.

A queda do número da categoria Não Sabe a partir de 2004 pode ter sido devido ao novo sistema TBWEB, implementado próximo a esse ano, o qual permite um acompanhamento do paciente a partir do diagnóstico. No entanto, seria esperado que um maior controle também implicasse na diminuição da categoria Pós-óbito, o que não ocorreu.

O box-plot (Figura 4.2) indica que, apesar da média das taxas de incidências estarem abaixo da incidência no estado, existe um tendência crescente nos dois últimos anos. A taxa de crescimento anual em todo o período analisado foi de $1,7 \%$ e os anos que apresentaram a maior e a menor taxa de incidência média foi em 2017 com 32,23 casos e em 2006 com 22,24 casos por 100 mil hab., 
Tipos de Entrada
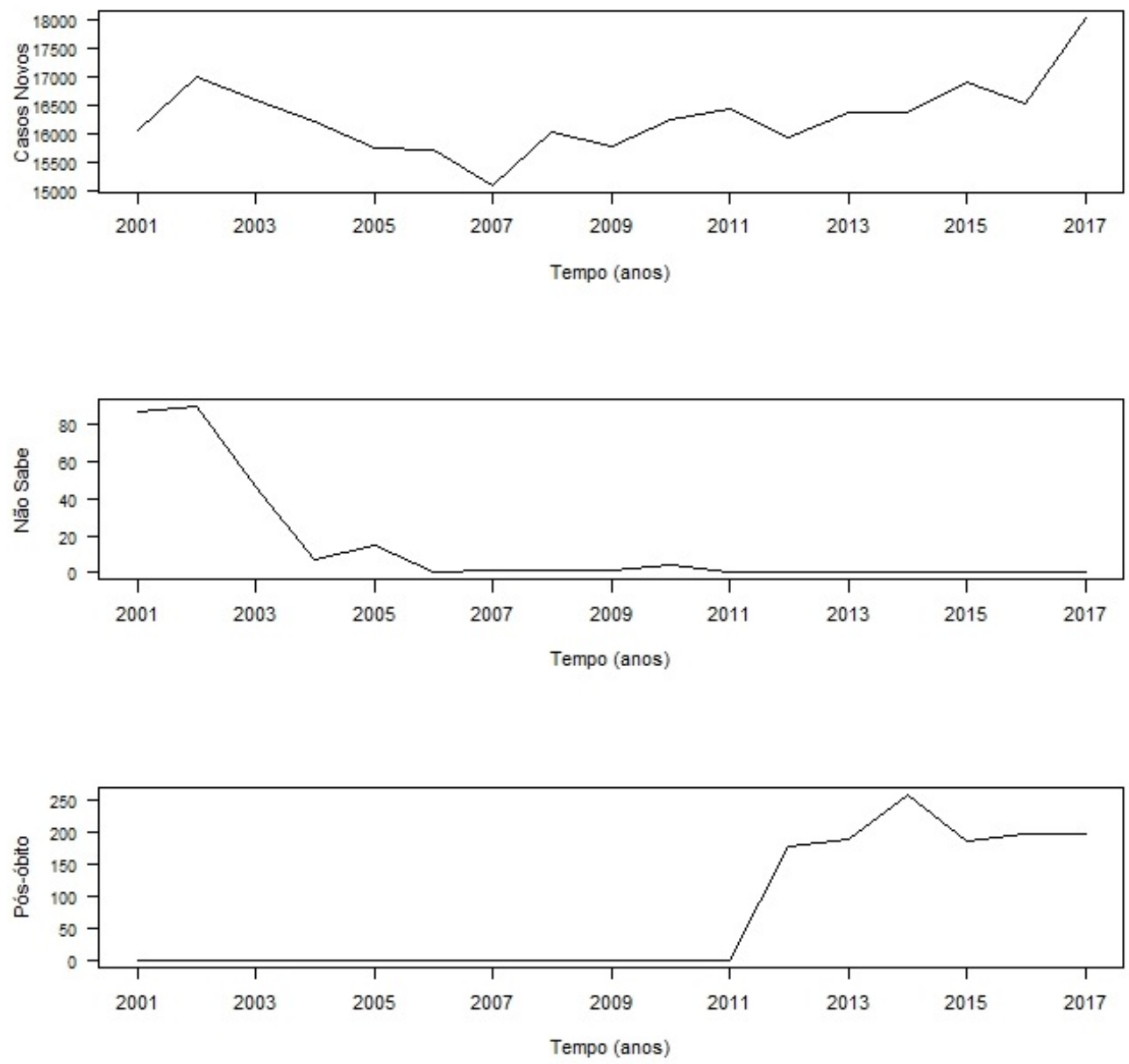

Figura 4.1: Variação dos dados brutos sobre Casos Novos, Não se Sabe e Pós-óbito registrados no período de 2001 até $201 \%$.

respectivamente. A maior taxa de incidência no estado foi no período de 2001 até 2003.

Os vinte municípios que apresentaram as taxas médias de incidência mais elevadas durante todo o período estão representados na Figura 4.3. O primeiro e o terceiro quantil para a média são 9,23 e 24,65 casos por 100 mil habitantes, indicando que 484 municípios possuem suas taxas médias menores que 25 casos por 100 mil habitantes. Apesar da expectativa de que a maioria dos municípios possuem baixos valores de incidência, 356 já tiveram suas taxas maiores que a média nacional de 40 casos por 100 mil habitantes em algum ano no período analisado.

O município de Borá apresentou uma taxa média de incidência altíssima comparada com os outros municípios. Isso aconteceu, pois a taxa de incidência é por 100 mil habitantes, sendo que esse município apresenta, em média, 826 habitantes $(95 \%, \mathrm{IC}=[815 ; 837])$, como houve 127 casos novos de tuberculose em 2005, correspondendo a $15 \%$ da população, o valor da incidência torna-se alto, quando extrapolado para uma população de 100 mil habitantes, chegando a uma taxa de 15431,34 casos por 100 mil hab. como mostra a Figura 4.4). Por esse motivo esse município foi considerado outlier. 


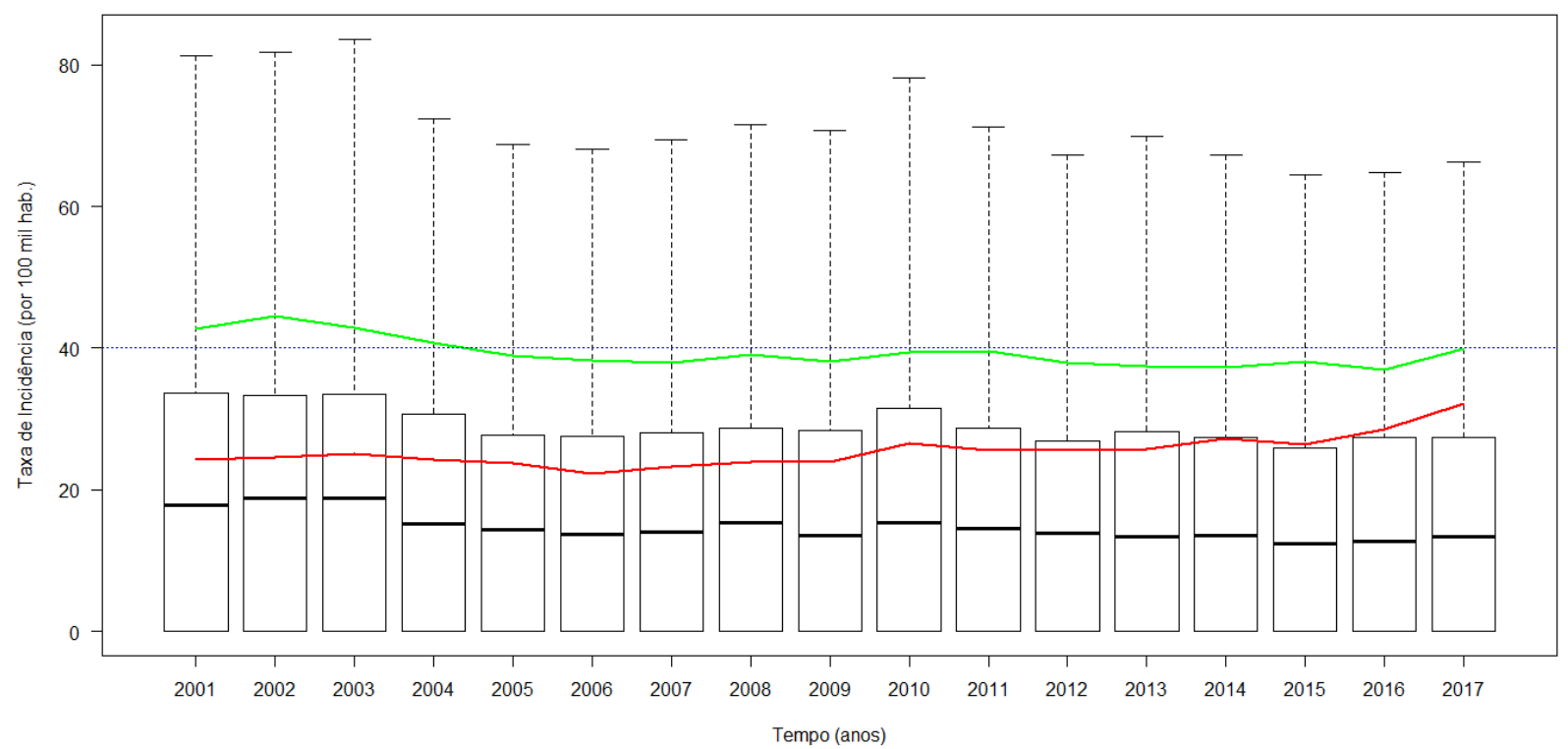

Figura 4.2: Box-plot da taxa de incidência dos municípios do estado de São Paulo com a taxa de incidência média no Brasil (linha tracejada em azul), taxa média de incidência no estado de São Paulo (linha sólida verde) e taxa média de incidência dos municípios (linha sólida vermelha), excluso o município de Borá e sem os outliers.

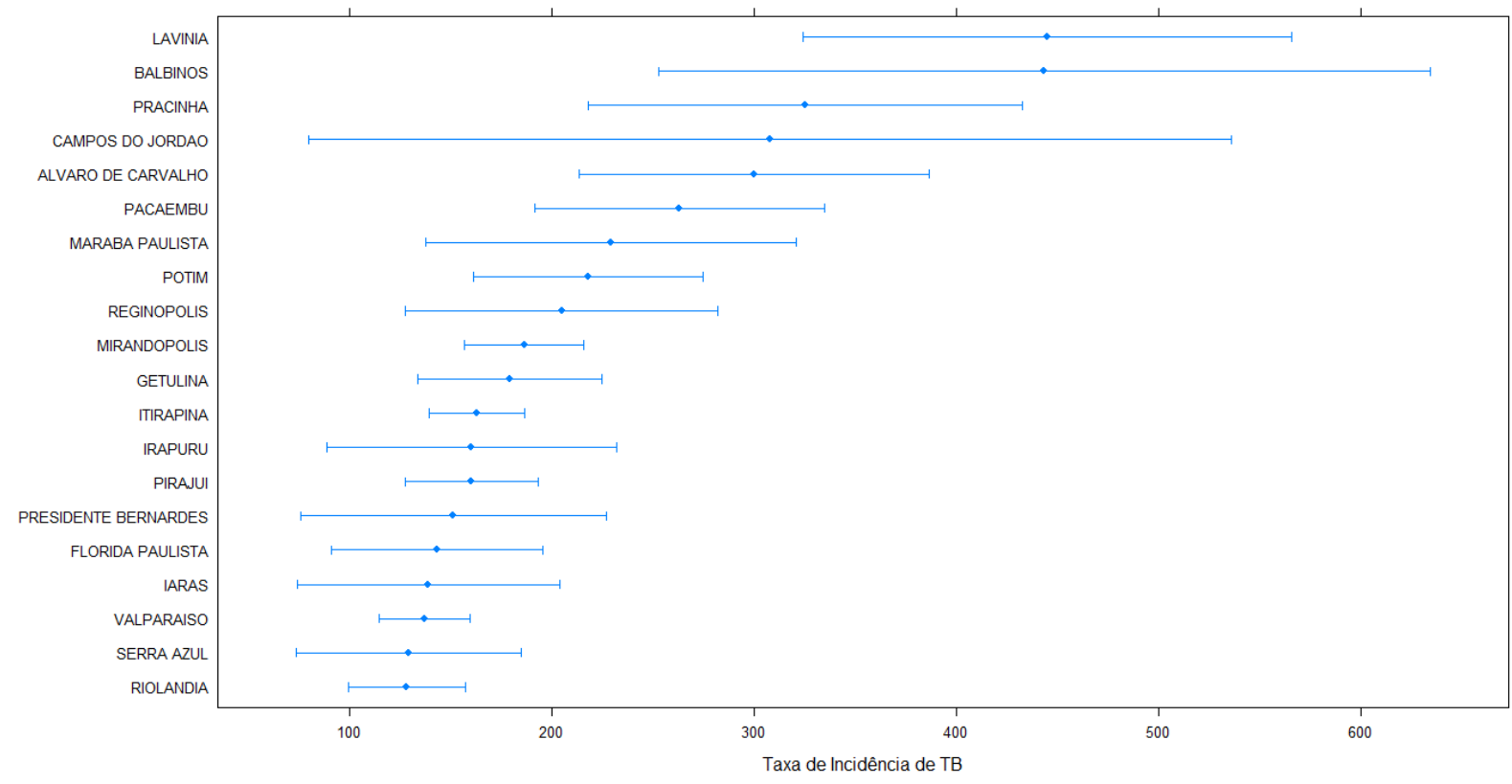

Figura 4.3: Os 20 municípios com as maiores taxas de incidência média registrada no período de 2001 até 2017 .

O município de Campos de Jordão apresentou a maior taxa de incidência em 2001 e foi identificado como outlier através das análises descritivas dos dados TS, esse município apresentou uma queda relativa de $99,19 \%$ no ano de 2017 , sendo que nesse mesmo ano foi registrado uma incidência de 9,71 casos por 100 mil habitantes. 
Taxa anual de íncidência de TB em Borá

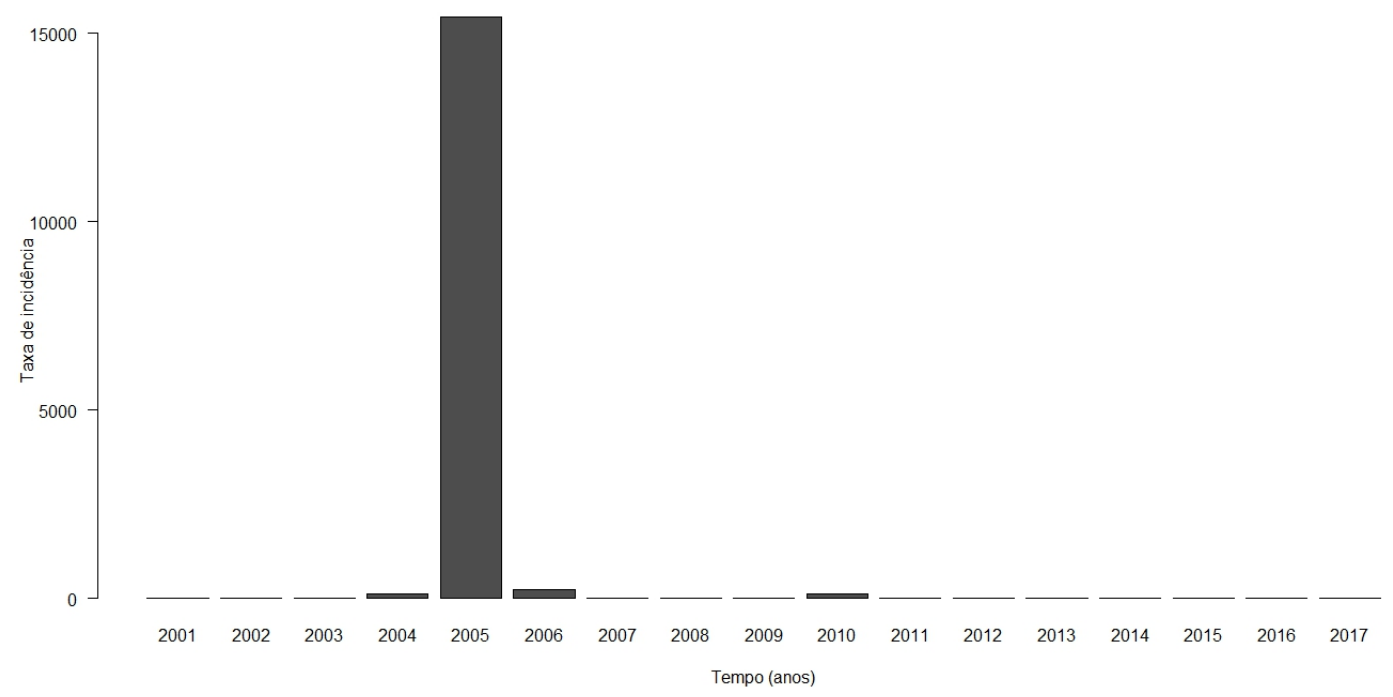

Figura 4.4: Taxa de incidência anual do município de Borá, no período de 2001 até 2017.
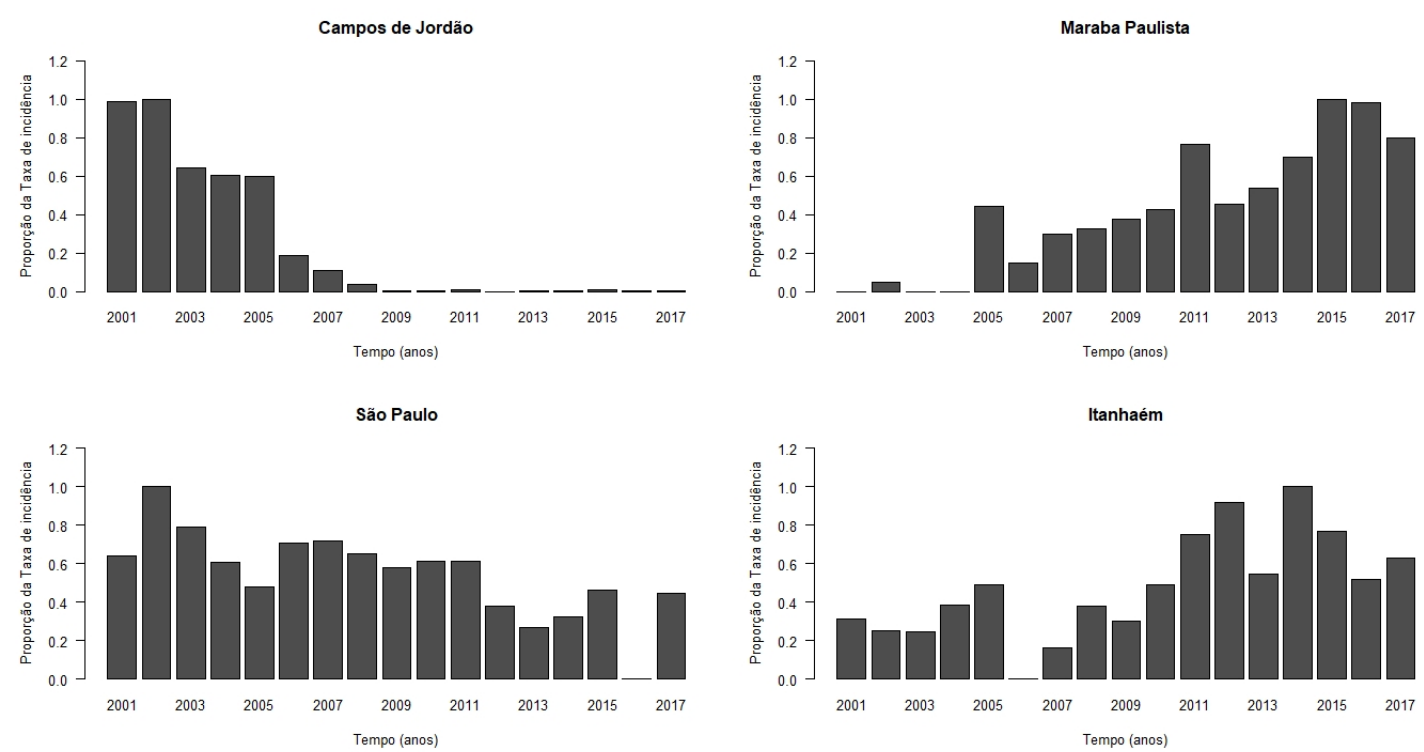

Figura 4.5: Proporção da taxas de incidência em quatro municípios que apresentaram comportamentos diferentes ao longo do tempo.

\subsection{Análise de Agrupamento nos dados TS}

Os municípios do estado de São Paulo apresentaram as mais variadas tendências em relação a doença analisada. A Figura 4.5 mostra alguns exemplos como em Campos de Jordão e Marabá Paulista com tendências decrescente e crescente, respectivamente, e em São Paulo e Itanhaém que oscilaram durante o período analisado, porém com comportamento decrescente e crescente, respectivamente, indicando uma heterogeneidade nas formas da série temporal para as diferentes localidades.

Utilizando os dados TS normalizados pelo método min-max e o algoritmo AGNES parametrizado com a distância entre grupos por average e a métrica adaptada de Spearman (equação 3.10), foram 
identificados dois grupos. A Figura 4.6 mostra os centróides e o box-plot anual de cada grupo. As duas séries temporais encontradas estão inversamente correlacionadas, com correlação linear de $0,83(95 \%, \mathrm{IC}=[-0,94 ;-0,60])$, evidenciando que há uma diferença entre as tendências, sendo que o grupo 1 indica uma tendência decrescente e o grupo 2 crescente.
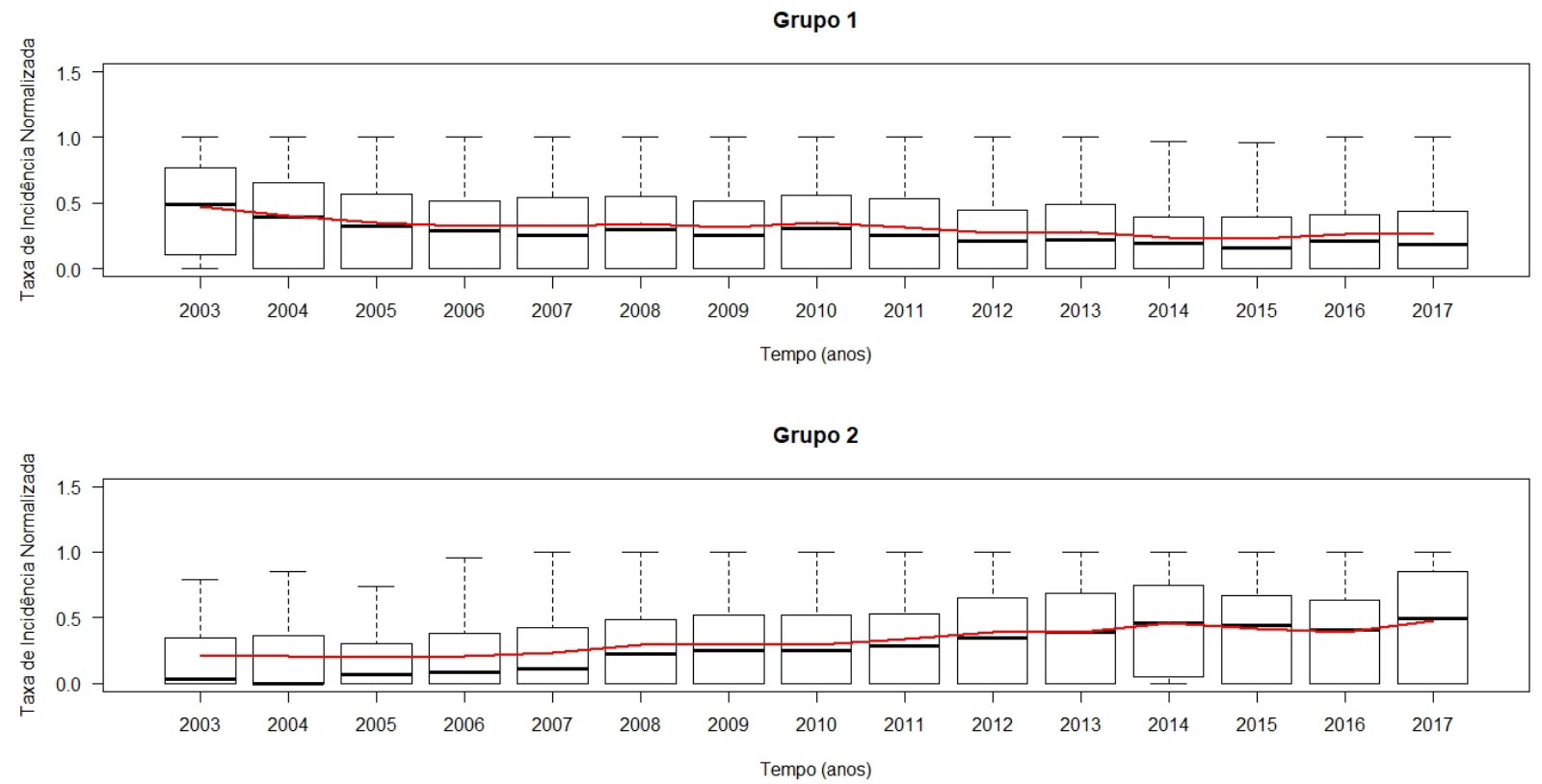

Figura 4.6: Box-plot das taxas de incidência normalizada e agrupadas segundo a metodologia proposta. A linha em vermelho é a média em cada ano.

A Figura 4.7 mostra as localidades agrupadas segundo a metodologia adotada neste trabalho. O grupo rotulado como zero são os municípios Arco-Íris, Buritizal, Cassia dos Coqueiros, Guatapará, Jambeiro, Marinópolis, Monteiro Lobato, Paulistânia, Quadra e Taquaral que não notificaram nenhum caso de tuberculose no período de estudo. As localidades nas mesorregiões de Araçatuba e Ribeirão Preto possui uma porção de municípios no grupo com tendência crescente (Grupo 2).

A Tabela 4.1 mostra em cada grupo a quantidade de municípios, a média do grupo e o intervalo de confiança utilizando o teste $t$ de Student. O resultado indica que aproximadamente $28 \%$ dos municípios possuem uma tendência crescente da incidência de tuberculose no período de 2001 até 2017.

\begin{tabular}{l|ccc}
\hline- & Grupo 0 & Grupo 1 & Grupo 2 \\
\hline \hline $\mathrm{N}^{\circ}$ de Municípios & 10 & 450 & 185 \\
Proporção & $1,55 \%$ & $69,76 \%$ & $28,68 \%$ \\
Média & 0 & 0,33 & 0,30 \\
$\mathrm{IC}$ & {$[0 ; 0]$} & {$[0,29 ; 0,37]$} & {$[0,24 ; 0,35]$} \\
\hline
\end{tabular}

Tabela 4.1: Número de municípios e as médias em cada grupo obtido pela execuçâo do algoritmo de agrupamento hierárquico. 
Clusters dos municípios do estado de São Paulo

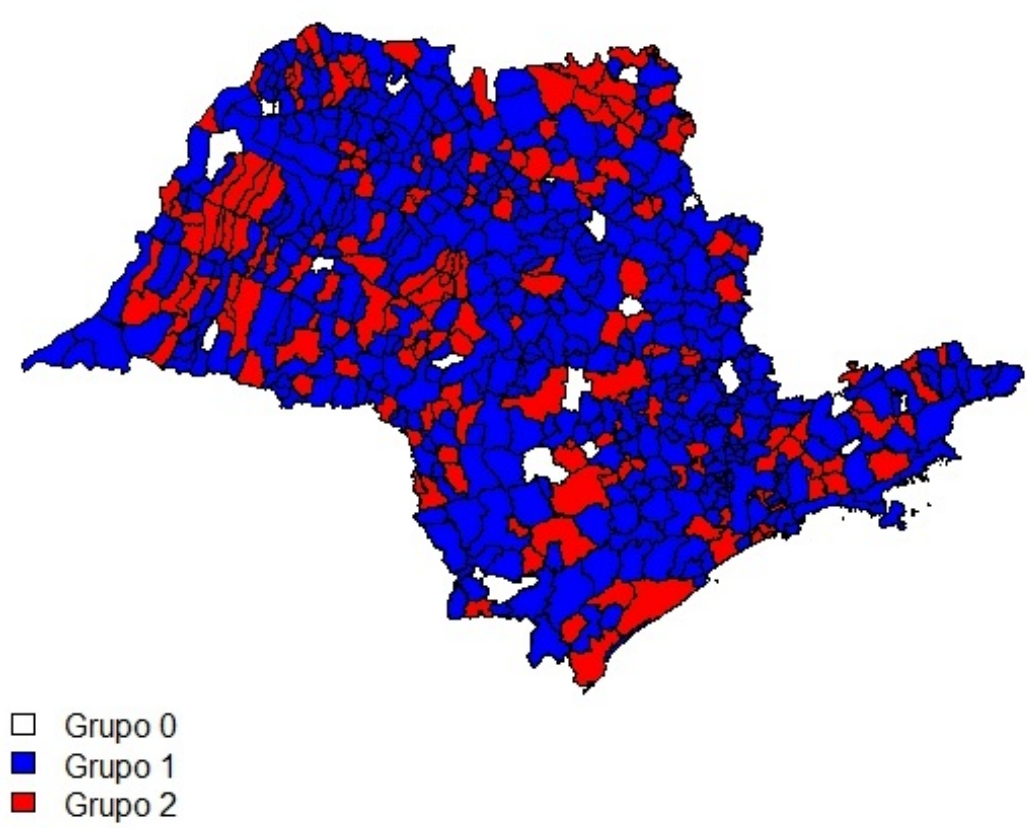

Figura 4.7: Agrupamento dos municípios de São Paulo gerado pelo algoritmo AGNES. 


\subsection{Análise Espacial dos Dados}

Os resultados mostram que durante todo o tempo houve uma dependência espacial significativa no estado (Figura 4.8), porém com os valores baixos nos índices, apresentando o menor e o maior IGM em 2001 com $I=0,113$ e p-valor em $1,34 \times 10^{-3}$ e em 2012 com $I=0,176$ e p-valor igual a $1,10 \times 10^{-10}$, respectivamente.

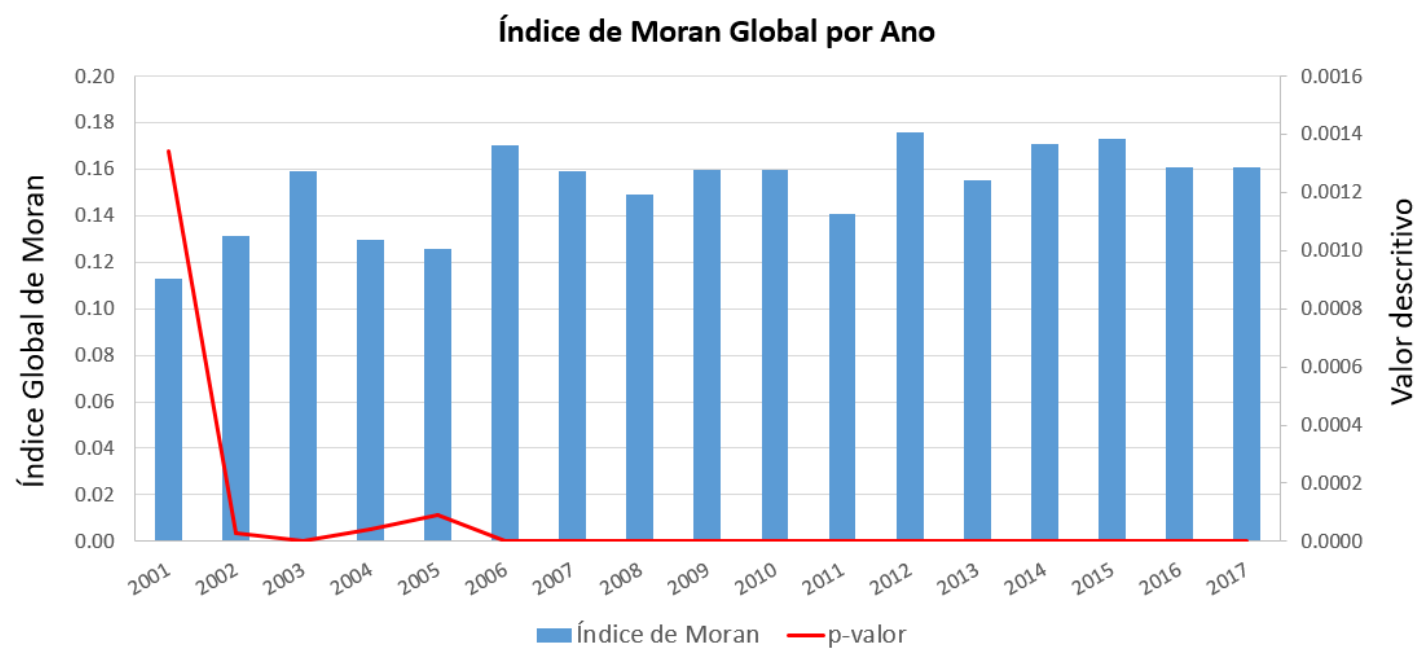

Figura 4.8: Gráfico de barras do indice global de Moran por ano no período analisado e seu valor descritivo obtido pelo método de Monte Carlo com 999 permutações dos municípios.

A Figura 4.9 mostra o mapa com os municípios de São Paulo que estão presentes nos quadrantes do diagrama de Moran cujo o índice local é significativo ( $\mathrm{p}$-valor $<5 \%$ ). Durante todo o período existem regiões que estão no quadrante Q1 (Alto-Alto) indicando que tais municípios presentes nessas regiões possuem valores altos e seus vizinhos também, revelando alto risco de tuberculose. Eles estão localizados próximos da costa litorânea, região de Bauru e em Presidente Prudente e Araçatuba. Já os municípios que estão no Q2 (Baixo-Baixo) variam com o tempo, mas revelando também que os riscos de tuberculose nesses locais são menores, sendo que a moda revela que esses municípios estão localizados na região de Ribeirão Preto.

Ao aplicar o Diagrama de Moran nos dados MI (Figura 4.10), a maior parte dos municípios encontra-se nos quadrantes Q2 (Baixo-Baixo) e Q4 (Baixo-Alto). O mapa construído a partir desse diagrama (Figura 4.11), indica que nas regiões norte e nordeste do estado possuem municípios com valores baixos e seus vizinhos também. Desse forma, é possível identificar grupos de municípios com o mesmo comportamento, gerando um padrão no estado. O índice global de Moran indica uma dependência espacial global de $0,252(95 \%, \mathrm{IC}=[0,211 ; 0,293])$ para os dados MI.

Ainda com base nos dados MI, a média da incidência nos 3 anos analisados é 28,98 casos por 100 mil hab. e o menor e maior valor registrado foi de 0 casos e 871,41 por 100 mil habitantes, respectivamente, sendo que o município de Balbinos apresentou o maior número de casos. Além disso, mais de 73 municípios estiveram acima da média nacional de 40 casos/100 mil habitantes.

Por meio do índice global de Moran, foi possível identificar as dependências espaciais das va- 

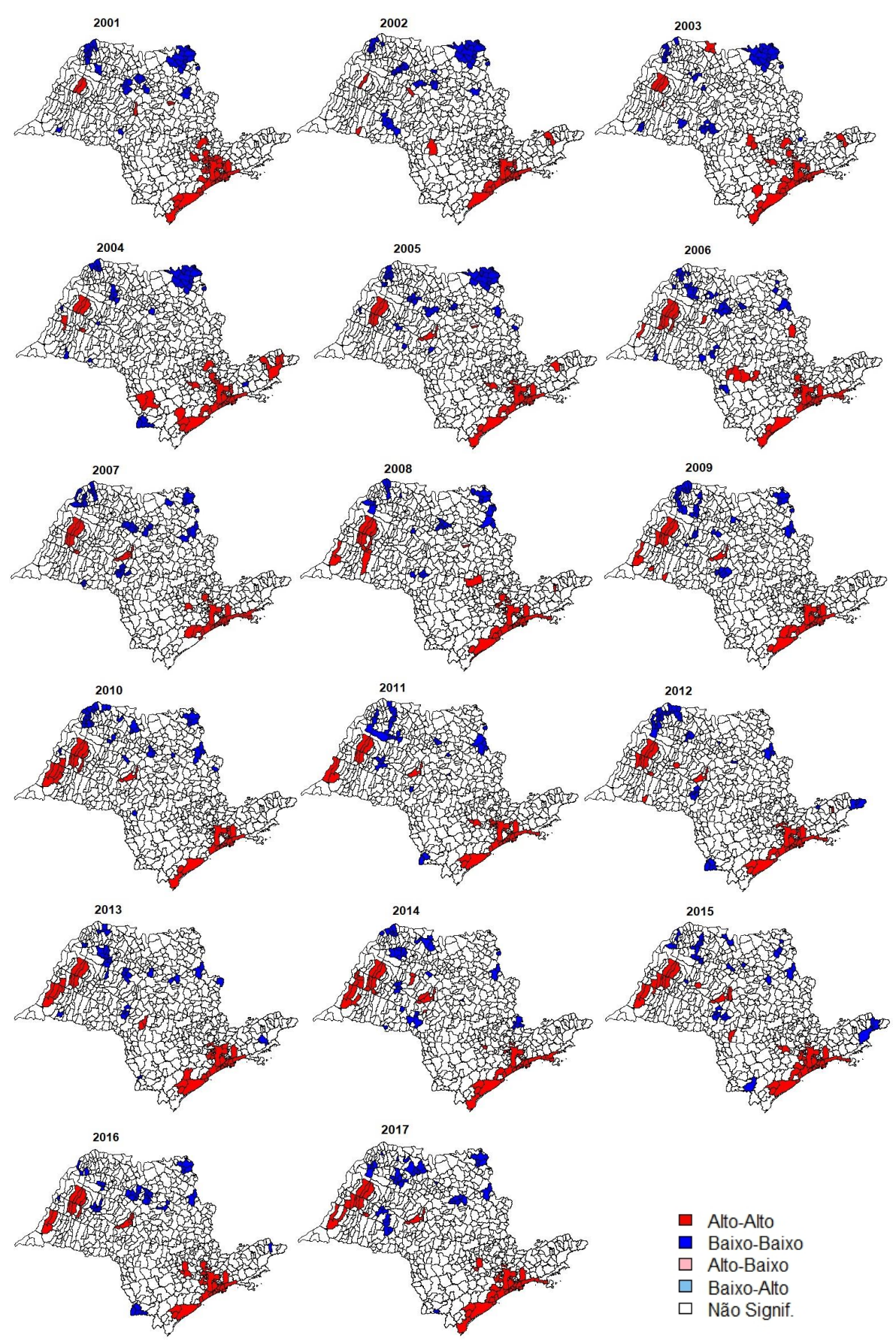

Figura 4.9: Mapa dos municípios de São Paulo com base nas classes do Diagrama de Moran obtidos pelo indice local que apresentaram correlações espaciais significativas ( $p$-valor $<5 \%$ ).

riáveis socioeconômicas na base de dados Socio. A Tabela 4.2 mostra os índices de Moran e os p-valores utilizando o método da pseudossignificância. Para um nível de significância de 5\%, as variáveis como distância ao litoral e presídios, densidade de pessoas por dormitório maior do que 2, taxa de roubo, taxa de técnicos de enfermagem registrados no COREN possuem dependência 
Diagrama de Moran

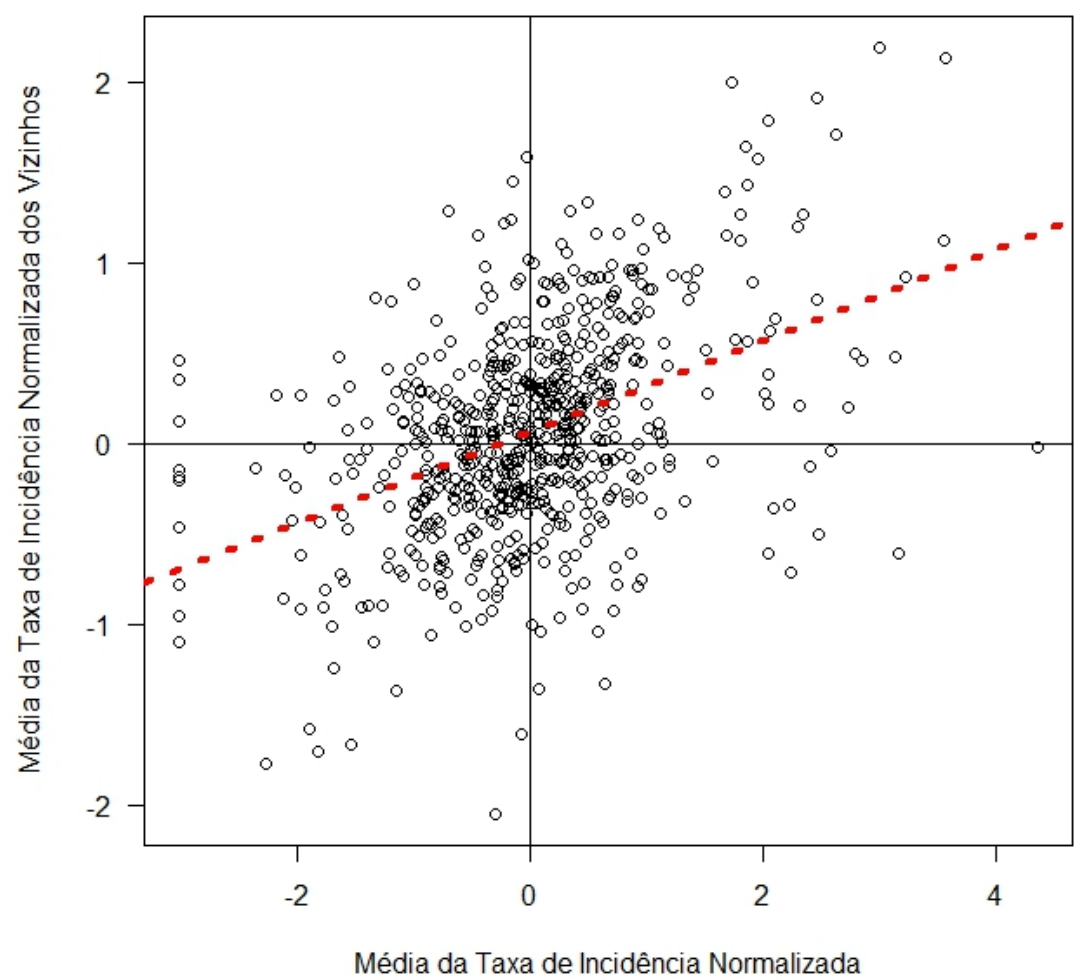

Figura 4.10: Diagrama de Moran da média das taxas de incidência no período de 2001 até 2017. A linha tracejada é a regressão linear feita pela média da taxa de incidência normalizada dos municípios e dos vizinhos.

espacial maiores do que 0,4 .

A única variável que possui dependência espacial negativa é a taxa de enfermeiros cadastrados no COREN, cujo o I de Moran é -0,051 (95\%, IC $=[-0,090 ;-0,011])$. O sinal negativo desse índice indica que, se o município tem valor alto, então seus vizinhos são baixos, ou vice-versa. Ao aplicar o Diagrama de Moran para cada variável socioeconômica (Figuras 4.12 e 4.13) foi possível identificar as regiões com essas dependências espaciais.

A variável que indica a distância ao litoral, possui uma dependência espacial muito forte. Além disso, relacionando com as sumarizações feitas nos dados socioeconômicos, a única variável que possui uma assimetria e curtose negativa é a distância ao litoral, revelando que a distribuição é leptocúrtica e com cauda pesada à direita, ou seja, há mais municípios longe da costa litorânea do que próximo dela.

Quanto a base de dados FX, a distribuição por faixas etárias não é uniforme (Tabela 4.3). A maioria dos municípios tem a categoria Idosos como a faixa etária mais atingida na prevalência média nos 3 últimos anos analisados. Cerca de 15,03\% dos municípios (97 localidades) não notificaram incidência de tuberculose nos últimos três anos. Apenas os municípios Alvinlandia e Patrocínio Paulista tiveram como faixa etária mais atingida a categoria Crianças, cujas taxas médias foram 


\section{Índice de Moran Local da média da Incidência}

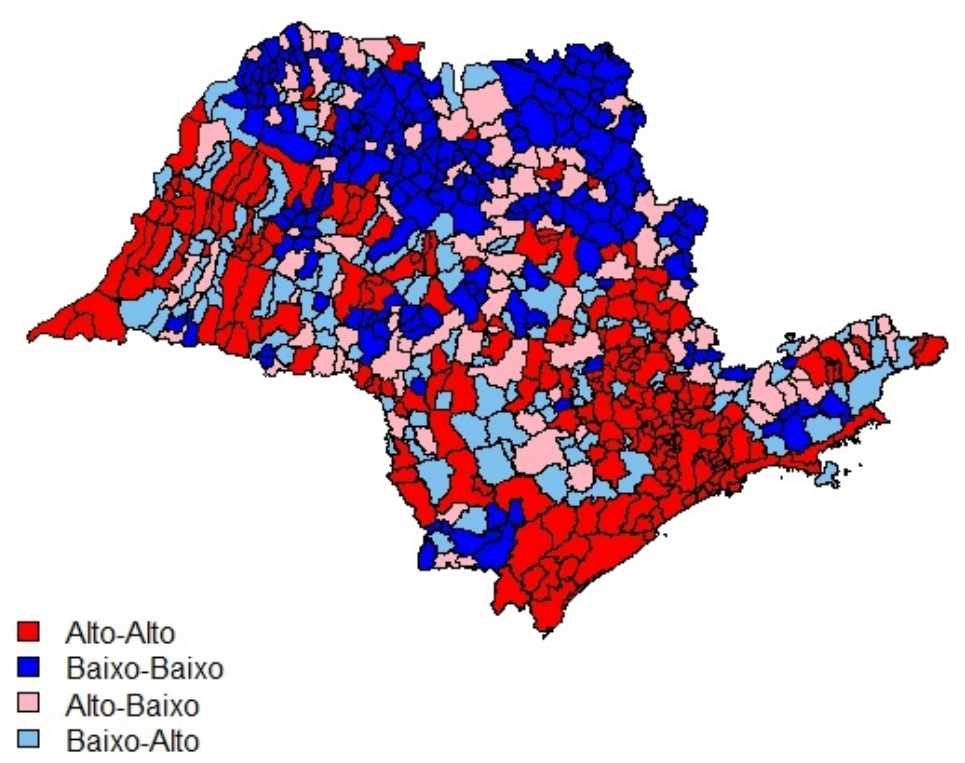

Figura 4.11: Mapa dos municípios que estão localizados nos quadrantes do diagrama de Moran.

\begin{tabular}{|c|c|c|c|c|}
\hline Atributos & I de Moran & Lower I & Upper I & p-valor \\
\hline DR_Desp_e_Lazer & 0,211 & 0,173 & 0,250 & 0 \\
\hline DR_Educacao & 0,099 & 0,064 & 0,133 & 0 \\
\hline$\overline{\mathrm{DR}}$ _Saude & 0,142 & 0,106 & 0,178 & 0 \\
\hline DR_Atencao_Basica & 0,168 & 0,128 & 0,208 & 0 \\
\hline DR_ĀAssitencia_Social & 0,203 & 0,163 & 0,243 & 0 \\
\hline DR_Transporte & 0,152 & 0,114 & 0,190 & 0 \\
\hline V_Homicidio_Doloso & 0,082 & 0,041 & 0,122 & 0 \\
\hline V_Estrupo & 0,235 & 0,192 & 0,278 & 0 \\
\hline $\mathrm{V}_{-}^{-}$Roubos & 0,751 & 0,713 & 0,788 & 0 \\
\hline V_Furtos_e-Roubos & 0,420 & 0,376 & 0,464 & 0 \\
\hline PS_Tecnico_de_Enfermagem & 0,411 & 0,369 & 0,452 & 0 \\
\hline PS_Medicos & 0,240 & 0,199 & 0,281 & 0 \\
\hline PS_Enfermeiros & $-0,051$ & $-0,090$ & $-0,011$ & 0,979 \\
\hline Taxa_de_Abandono_no_EF & 0,197 & 0,154 & 0,24 & 0 \\
\hline Taxa_de_Abandono_no_EM & 0,171 & 0,129 & 0,212 & 0 \\
\hline $\mathrm{ID} H \mathrm{H}$ & 0,248 & 0,203 & 0,292 & 0 \\
\hline Dens_Dormitorio_mais_de_2 & 0,670 & 0,633 & 0,706 & 0 \\
\hline$\overline{\text { Dist_presidio }}$ & 0,824 & 0,799 & 0,849 & 0 \\
\hline Dist_litoral & 0,971 & 0,959 & 0,983 & 0 \\
\hline
\end{tabular}

Tabela 4.2: Índice Global de Moran para as variáveis socioeconômicas.

7,15 por 100 hab. e 1,67 por 100 hab., respectivamente. Para a faixa etária Adolescentes, 23 municípios notificaram essa faixa etária como a mais atingida, sendo que o município de Reginópolis apresentou uma prevalência de 79,03 casos por 100 habitantes nessa categoria. 

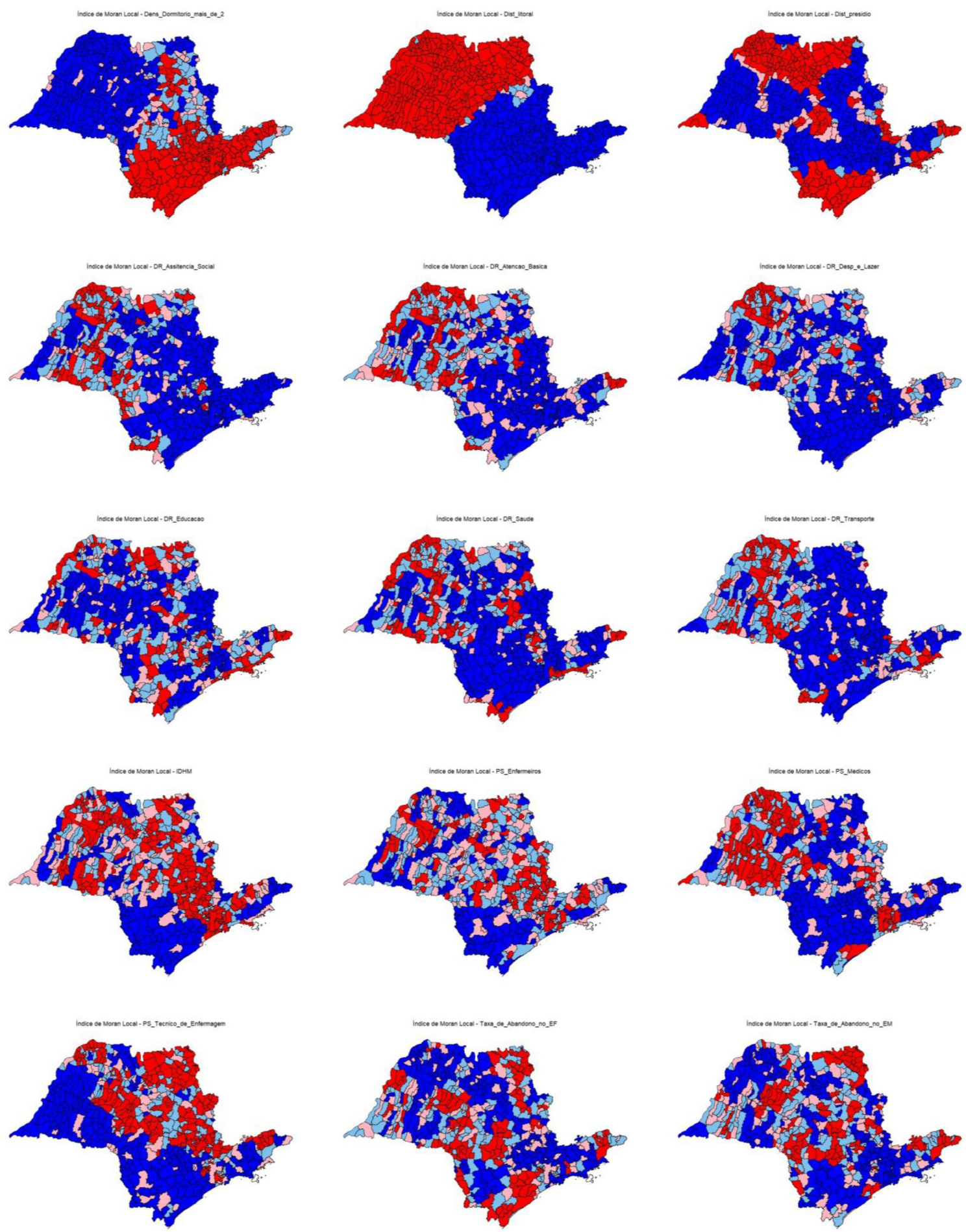

Figura 4.12: Parte 1: Mapa dos municípios de São Paulo após a utilização do método do diagrama de Moran.

\begin{tabular}{l|ccccc}
\hline- & Crianças & Adolescentes & Adultos & Idosos & Nenhum \\
\hline \hline $\mathrm{N}^{\circ}$ de Municípios & 2 & 23 & 80 & 443 & 97 \\
Proporção & $0,31 \%$ & $3,56 \%$ & $12,40 \%$ & $68,68 \%$ & $15,03 \%$ \\
\hline
\end{tabular}

Tabela 4.3: Proporções de municípios segundo as faixas etárias obtidas dos dados FX. 

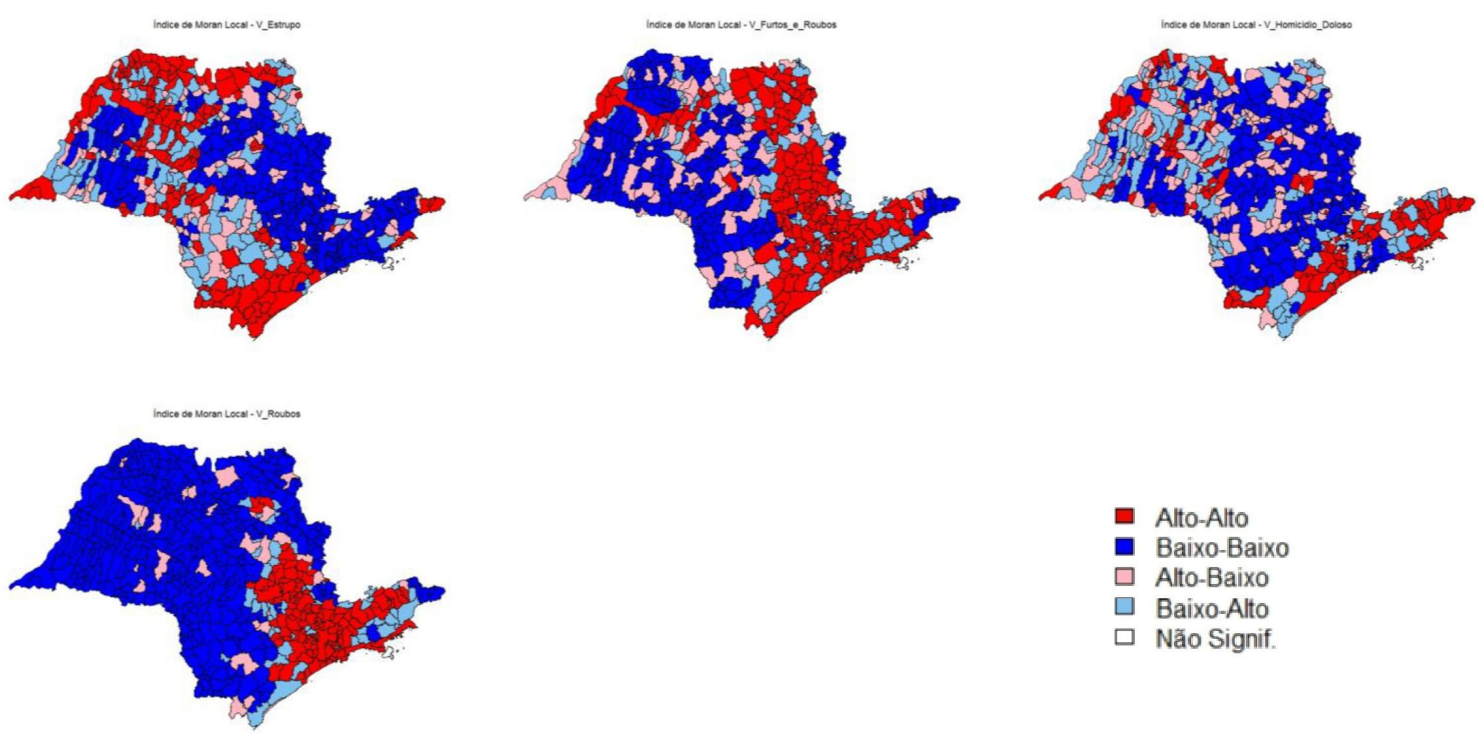

Figura 4.13: Parte 2: Mapa dos municípios de São Paulo após a utilização do método do diagrama de Moran.

Com base na Tabela 4.3, observa-se que as duas faixas etárias que apresentaram a maior quantidade de municípios foram Adultos e Idosos, sendo esse último com cerca de $68 \%$ dos municípios nessa categoria, revelando que a doença atinge, principalmente, os idosos. Os locais que notificaram as maiores prevalências nessas duas faixas etárias foi em Presidente Bernardes com 70,83 casos por 100 hab. e em Balbinos com 647 casos por 100 habitantes. O mapa 4.14 mostra os municípios e as categorias das faixas etárias mais atingidas segundo a prevalência média nos últimos 3 anos analisados.

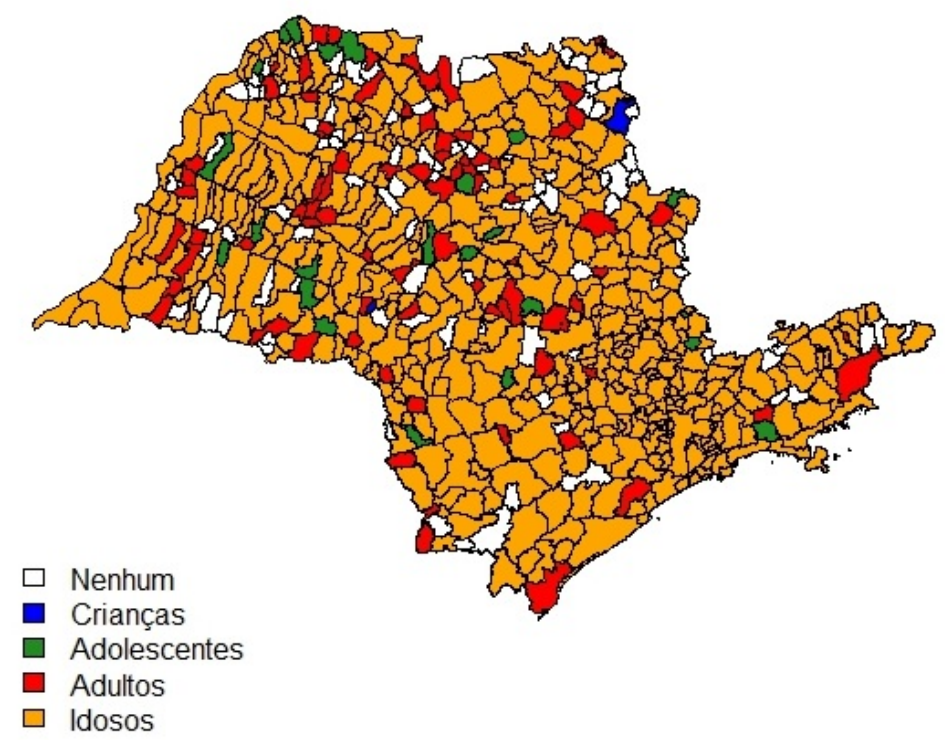

Figura 4.14: Categorias dos dados FX nos municípios de São Paulo. 


\subsection{Extração dos Padrões}

A seguir serão apresentados as regras extraídas no conjunto de dados com o uso do algoritmo APRIORI e utilizando diferentes valores para o suporte e confiança. Além das regras de associação entre as variáveis socioeconômicas, relações entre as variáveis envolvendo a doença e os dados socioeconômicos também foram obtidos. Para cada regra analisada um mapa com as localizações de cada município foi gerado.

\section{Tendência da incidência e MI}

R1:

$$
\begin{gathered}
\{\mathrm{O} 2=\text { Baixo-Baixo, O4=Alto-Alto, } \mathrm{MI}=\text { Alto-Alto }\}=>\{\text { Grupos }=2\} \\
\text { Sup. }=0,03 ; \text { Conf. }=0,90 ; \text { Lift }=3,16 ; \text { Conv. }=7,84 ; \mathrm{RI}=0,03 ; \text { Cont. }=20
\end{gathered}
$$

\section{Municípios pertencentes a regra $\mathrm{R} 1$}

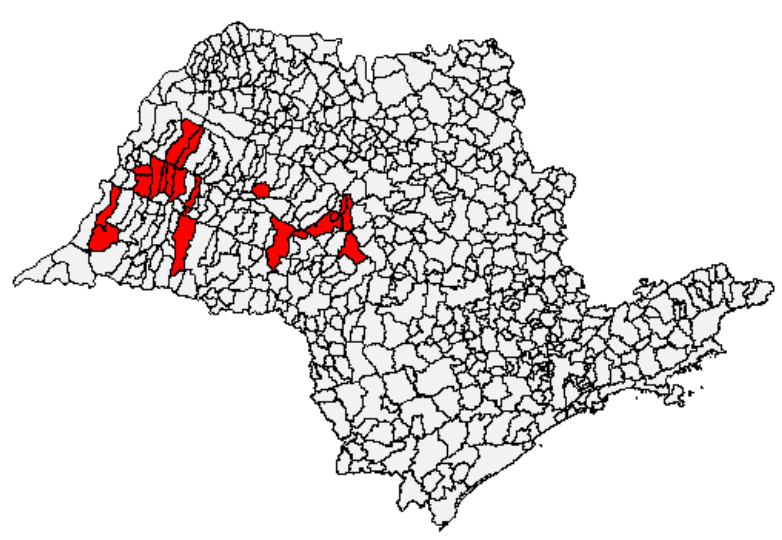

Figura 4.15: Os municípios em destaque pertencem a regra $R 1$.

Essa regra mostra que para os municípios longe da costa litorânea e com baixa densidade de moradores por dormitório, se a taxa de incidência for alta, então a tendência é crescente com $90 \%$ de confiança. Essa regra apresentou o maior valor de conviç̧ão e lift dentre as próximas 5 regras buscadas. A convicção de 7,84 indica que a probabilidade de ocorrer o consequente sem que o antecedente aconteça é de 7,84 vezes menor do que o esperado.

Cerca de 20 municípios se enquadram nesta regra. A Figura 4.15 mostra as localizações espaciais dos municípios pertencentes a regra $\mathrm{R} 1$. 
R2:

$$
\begin{gathered}
\{\mathrm{D} 1=\text { Baixo-Baixo, } \mathrm{S} 1=\text { Baixo-Baixo, Grupos }=2\}=>\{\mathrm{MI}=\text { Alto-Alto }\} \\
\text { Sup. }=0,03 ; \text { Conf. }=0,40 ; \text { Lift }=4,24 ; \text { Conv. }=1,52 ; \mathrm{RI}=0,02 ; \text { Cont }=20
\end{gathered}
$$

\section{Municipios pertencentes a regra $\mathrm{R} 2$}

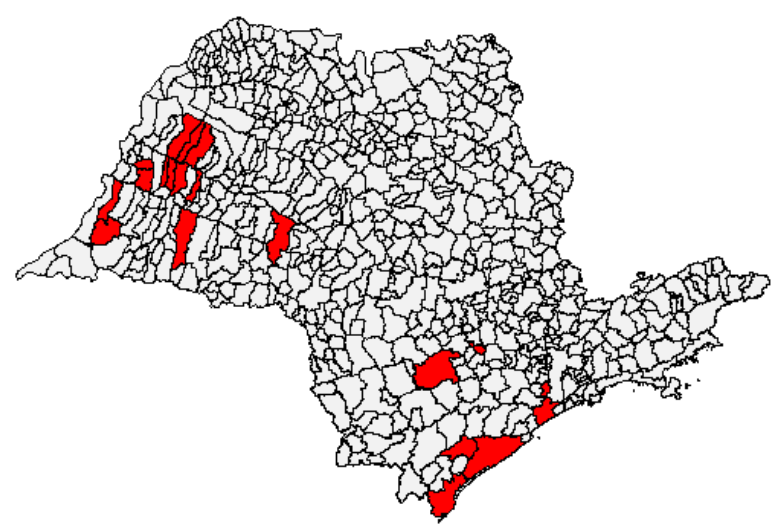

Figura 4.16: Os municípios em destaque pertencem a regra $R 2$.

A regra R2 mostra que se a despesa com laser e desporto for baixo, técnicos de enfermagem registrado no COREN for baixo, com o mesmo padrão ocorrendo aos seus vizinhos e a tendência for crescente na incidência de tuberculose, então a taxa de incidência nos três últimos meses também serão altos. Essa regra possui o lift de 4,24, indicando uma dependência positiva, pois o suporte é 4,24 vezes maior do que o suporte esperado. Contudo, essa regra possui a menor confiança registrada (40\% da confiança). A Figura 4.16 mostra as localizações espaciais dos municípios.

R3:

$\{\mathrm{V} 5=$ Baixo-Baixo, O3=Alto-Alto, $\mathrm{O} 4=$ Alto-Alto, Grupos $=2\} \Rightarrow>$ MI $=$ Baixo-Baixo $\}$ Sup. $=0,06$; Conf. $=0,86 ;$ Lift $=1,39 ;$ Conv. $=2,84 ; \mathrm{RI}=0,02 ;$ Cont. $=39$

R4:

$$
\begin{gathered}
\{\mathrm{D} 16=\text { Baixo-Alto, O3=Alto-Alto, Grupos }=1\}=>\{\mathrm{MI}=\text { Baixo-Baixo }\} \\
\text { Sup. }=0,05 ; \text { Conf. }=1 ; \text { Lift }=1,61 ; \text { Conv. }=\mathrm{NA} ; \mathrm{RI}=0,02 ; \text { Cont } .=36
\end{gathered}
$$

As regras R3 e R4 possuem os mesmos consequentes e, apesar dos antecedentes aparecerem duas categorias diferentes (Grupos=1 e Grupos=2), a regra R4 indicou a maior confiança. Essa última regra diz que se a despesa com assistência social for baixa e o mesmo ocorrer ao seus vizinhos, estando distante dos presídios, caso a tendência da incidência de tuberculose seja decrescente, então a incidência média nos três últimos anos analisados será baixo nesses municípios e nos seus vizinhos. Essas regras possuem RI igual a 0,02, indicando que há uma dependência positiva do antecedente para o consequente. Os municípios presentes nestas regras estão ilustrados nas Figuras 4.17 e 4.18. 
Municípios pertencentes a regra R3

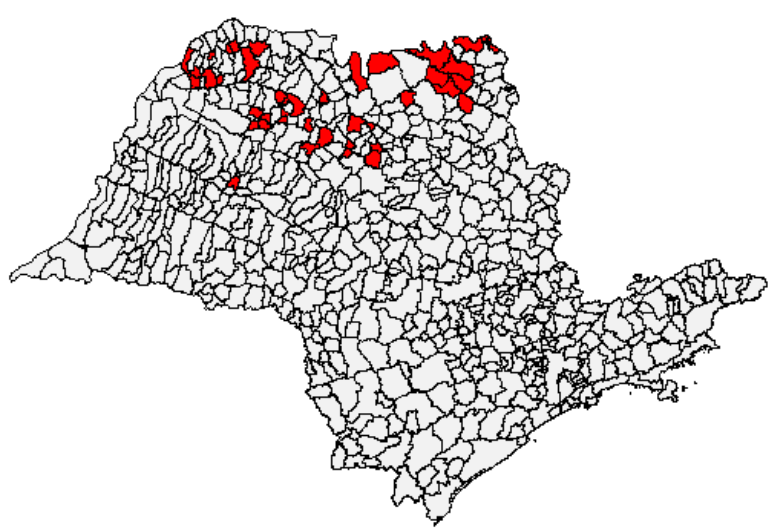

Figura 4.17: Os municípios em destaque pertencem a regra $R 3$.

Municípios pertencentes a regra R4

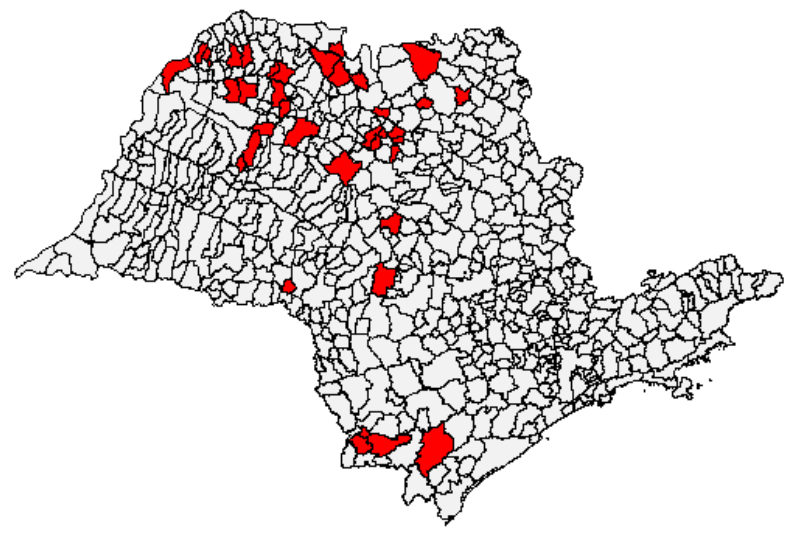

Figura 4.18: Os municipios em destaque pertencem a regra $R 4$. 
R5:

$\{\mathrm{S} 3=$ Alto-Alto, $\mathrm{A} 1=$ Baixo-Baixo, $\mathrm{O} 4=$ Baixo-Baixo, $\mathrm{MI}=$ Baixo-Baixo $\}=>\{$ Grupos $=1\}$

Sup. $=0,03 ;$ Conf. $=1$; Lift $=1,43 ;$ Conv. $=\mathrm{NA} ; \mathrm{RI}=0,01 ;$ Cont.$=22$

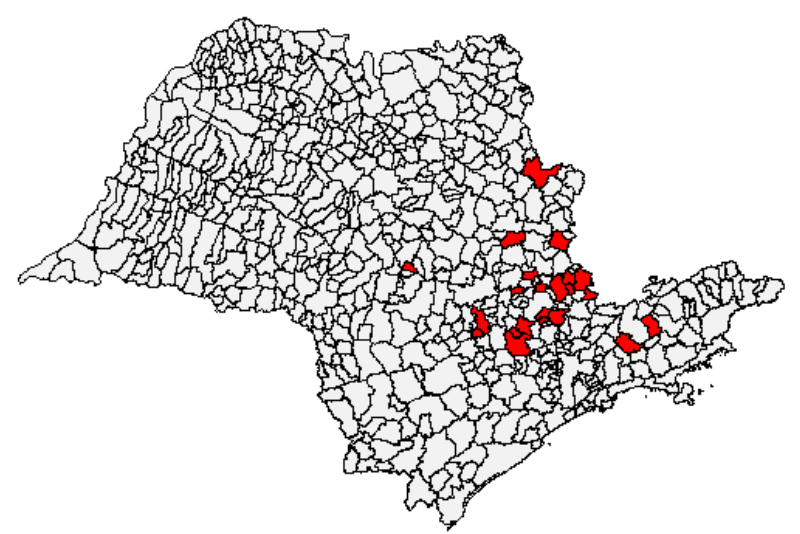

Figura 4.19: Os municípios em destaque pertencem a regra $R 5$.

R6:

$\{$ V3 $=$ Baixo-Baixo, $\mathrm{O} 1=$ Alto-Alto, $\mathrm{MI}=$ Baixo-Baixo $\}=>\{$ Grupos $=1\}$

Sup. $=0,10 ;$ Conf. $=0,85 ;$ Lift $=1,22 ;$ Conv. $=2,01 ; \mathrm{RI}=0,01 ;$ Cont. $=68$

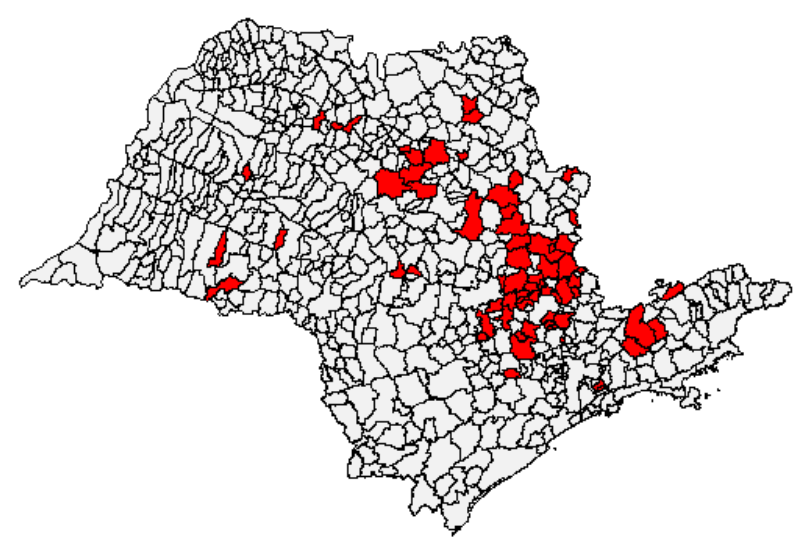

Figura 4.20: Os municípios em destaque pertencem a regra $R 6$.

As regras R5 e R6 foram extraídas fixando o antecedente em MI=Baixo-Baixo obtendo-se os mesmos consequentes. Entretanto, o Lift da regra R5 é maior do que R6, indicando que R5 possui mais independência entre o consequente e o antecedente. Entretanto, a convicção de R5 não 
está definida, pois há uma confiança de 100\% nessa regra, não sendo possível analisar a convicção. Apesar disso, R5 é mais interessante do que R6, porém esta última regra não deixa de mostrar sua importância relacionando as variáveis IDHM e taxa de estupro com a incidência de tuberculose. Os municípios pertencentes a regra R6 estão ilustrados na Figura 4.19.

A regra R5 indica que se o número de enfermeiros registrados no COREN for alto, a taxa de abandono no EF for baixa, possuindo uma taxa de incidência baixa e o mesmo comportamento ocorre aos vizinhos, se estiver longe da costa litorânea, então a tendência da incidência de tuberculose será decrescente (Figura 4.20).

\section{Faixa etária mais atingida e dados socioeconômicos}

R7:

$$
\begin{gathered}
\{\mathrm{D} 9=\text { Baixo-Baixo }\} \Rightarrow\left\{\mathrm{FX}_{-} \max =\mathrm{Idosos}\right\} \\
\text { Sup. }=0,34 ; \text { Conf. }=0,84 ; \mathrm{Lift}=1,22 ; \text { Conv. }=1,98 ; \mathrm{RI}=0,06 ; \text { Cont. }=224
\end{gathered}
$$

\section{Municípios pertencentes a regra $\mathrm{R} 7$}

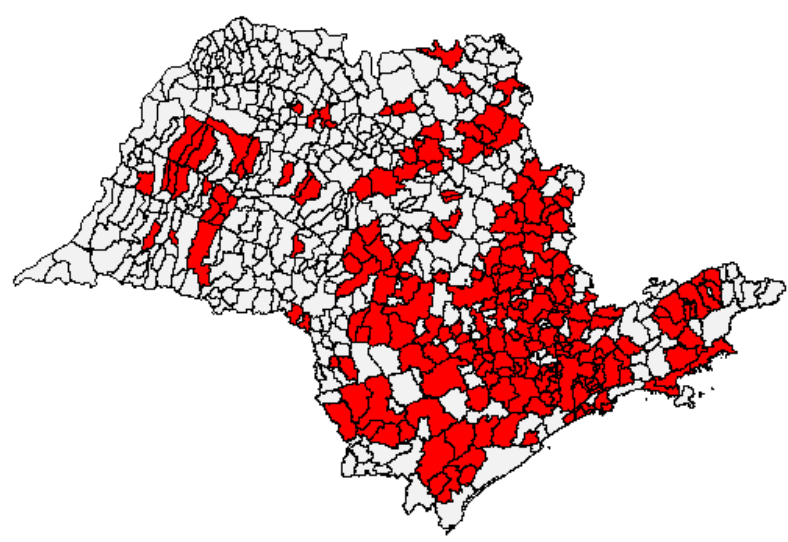

Figura 4.21: Os municipios em destaque pertencem a regra $R \%$.

R8:

$$
\begin{gathered}
\{\mathrm{D} 8=\text { Baixo-Baixo,O2=Alto-Alto, } \mathrm{MI}=\text { Alto-Baixo }\}=>\left\{\mathrm{FX} \_\max =\mathrm{Idosos}\right\} \\
\text { Sup. }=0,03 ; \text { Conf. }=1 ; \text { Lift }=1,45 ; \text { Conv. }=\mathrm{NA} ; \mathrm{RI}=0,01 ; \text { Cont. }=21
\end{gathered}
$$

As duas regras relacionam a faixa etária mais atingida com os dados socioeconômicos e MI. A regra $\mathrm{R} 7$ possui o maior suporte, indicando que em 224 municípios se a despesa com atenção básica foi baixa e o mesmo ocorrer aos seus vizinhos, então a faixa etária mais atingida será os idosos com $84 \%$ de confiança (Figura 4.21). A convicção registrada indica que a probabilidade do consequente ocorrer sem que o antecedente ocorra é quase 2 vezes menor do que o esperado. Contudo, a regra mais forte é R8, indicando que se a despesa com saúde for baixa e a densidade de moradores por 


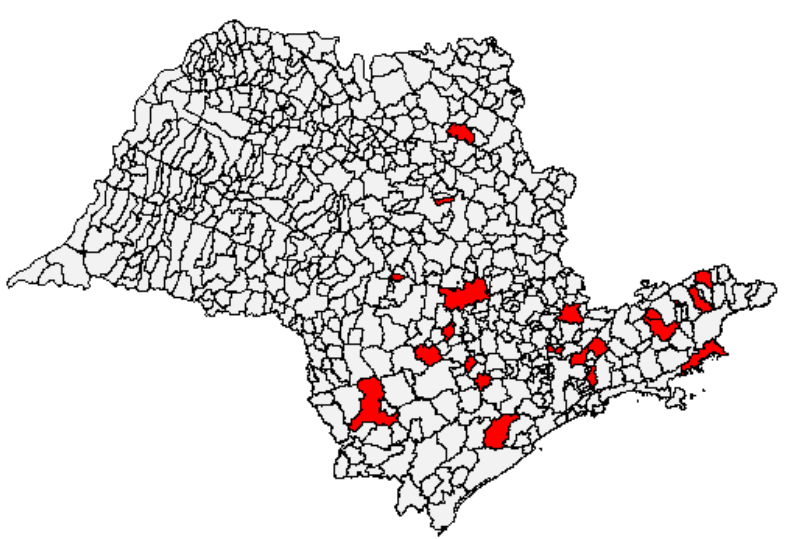

Figura 4.22: Os municípios em destaque pertencem a regra $R 8$.

dormitório for alto, ocorrendo o mesmo aos vizinhos, e a incidência nos três últimos anos for alta, não seguindo o mesmo padrão aos vizinhos, então a faixa etária mais atingida são os idosos com $100 \%$ de confiança nos 21 municípios registrados (Figura 4.22).

\section{Densidade de moradores por dormitório e MI}

R9:

$$
\begin{gathered}
\{\mathrm{D} 8=\text { Baixo-Baixo, } \mathrm{O} 4=\text { Baixo-Baixo, } \mathrm{MI}=\text { Alto-Alto }\}=>\{\mathrm{O} 2=\text { Alto-Alto }\} \\
\text { Sup. }=0,03 ; \text { Conf. }=1 ; \text { Lift }=3,05 ; \text { Conv. }=\mathrm{NA} ; \mathrm{RI}=0,03 ; \text { Cont. }=22
\end{gathered}
$$

Apesar da regra R8 mostrar que as variáveis D8, O2 e MI estão relacionados, a regra R9 apareceu novamente essas categorias, porém com a variável O4 inserida. Essa regra indica que se a despesa com saúde for baixa, a incidência de tuberculose for baixa e o mesmo ocorrer aos seus vizinhos, estando próximos a costa litorânea, então a densidade de moradores por dormitório será alta e os seus vizinhos também, com $100 \%$ de confiança e dependência positiva, pois o suporte da regra é 3,05 vezes maior do que o esperado (Figura 4.23).

\section{IDHM e MI}

R10:

$\{\mathrm{D} 1=$ Baixo-Baixo, D16=Baixo-Baixo, V5=Alto-Alto, O1=Alto-Alto, O2=Alto-Alto, $\mathrm{O} 4=$ Baixo-Baixo $\} \Rightarrow\{\mathrm{MI}=$ Alto-Alto $\}$

$$
\text { Sup. }=0,03 ; \text { Conf. }=0,45 ; \text { Lift }=4,77 \text { Conv. }=1,68 ; \mathrm{RI}=0,02 ; \text { Cont. }=17
$$

Os municípios que se enquadram na regra R10 são aqueles próximos da costa litorânea e que possuem IDHM alto, alta densidade de moradores por dormitório, baixa despesa com assistência 


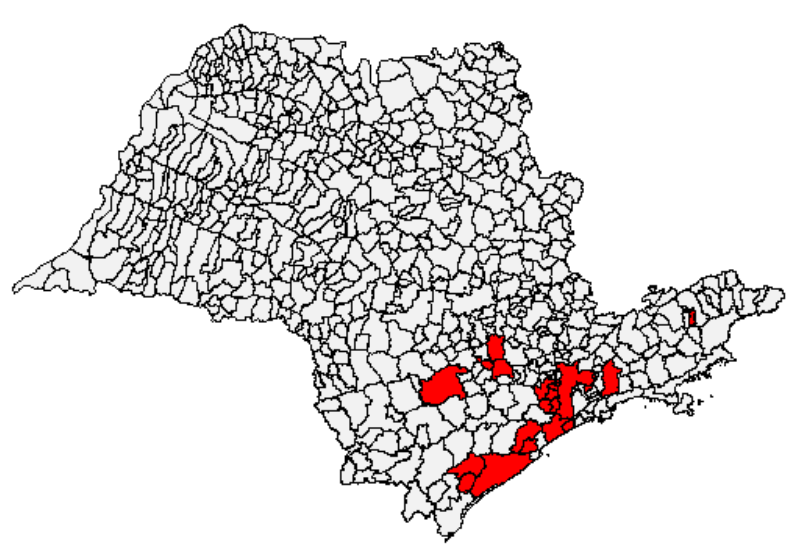

Figura 4.23: Os municipios em destaque pertencem a regra $R 9$.

\section{Municípios pertencentes a regra $\mathrm{R} 10$}

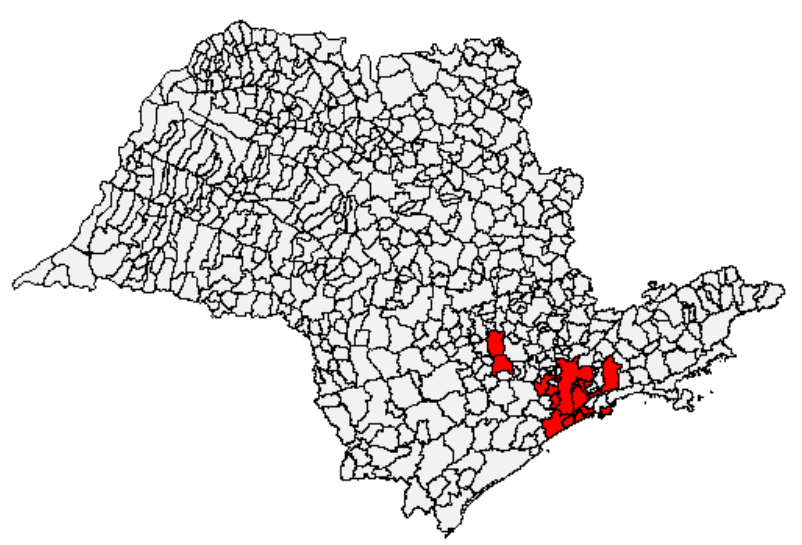

Figura 4.24: Os municipios em destaque pertencem a regra $R 10$.

social e com desporto e lazer, sendo que esse mesmo comportamento ocorre aos seus vizinhos, então a taxa de incidência nessas localidades é alta (Figura 4.24). Cerca de 21 municípios estão nessa regra e a convicção de 1,68 mostra a força de independência entre o antecedente e o consequente.

\section{Litoral, presídios e tendência de tuberculose}

R11:

$$
\begin{gathered}
\{\mathrm{V} 5=\text { Baixo-Baixo, O3=Alto-Alto }\}=>\{\mathrm{MI}=\text { Baixo-Baixo }\} \\
\text { Sup. }=0,26 ; \text { Conf. }=0,90 ; \text { Lift }=1,44 ; \text { Conv. }=3,73 ; \mathrm{RI}=0,08 ; \text { Cont. }=168
\end{gathered}
$$

A regra R11 mostra que as localidades e seus vizinhos com baixa taxa de violência com roubo e distantes dos presídios, teve a média da taxa de incidência baixa, com $90 \%$ de confiança ocorrendo 


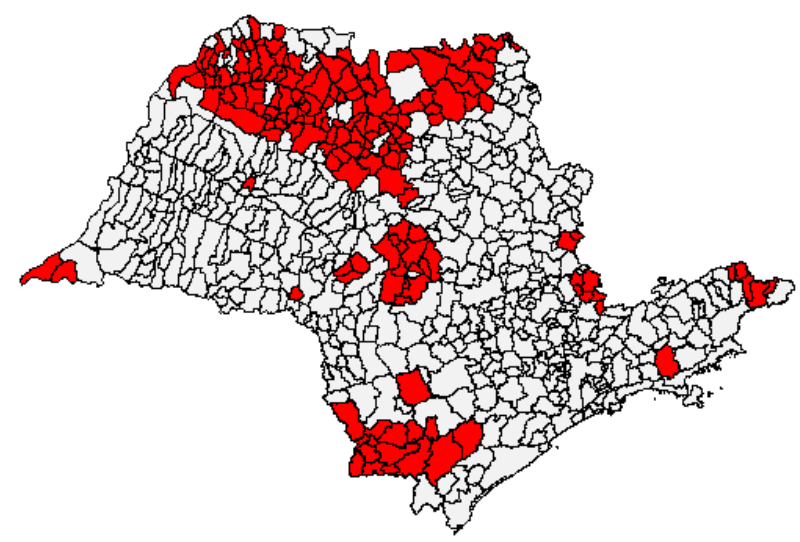

Figura 4.25: Os municipios em destaque pertencem a regra $R 11$.

em 168 municípios do estado de São Paulo (Figura 4.25). A diferença do suporte esperado para o real é de $8 \%$, mostrando que há uma dependência positiva. Além disso, a probabilidade de ocorrer o consequente sem que o antecedente aconteça é de 3,73 vezes maior do que o esperado.

Utilizando os mesmos parâmetros descritos acima, não houve nenhuma regra com os consequentes FX_max=Adolescentes ou FX_max=Crianças, isso porque a frequência relativa com que aparece essas categorias é menor do que $3 \%$.

A Tabela 4.4 possui os 40 municípios classificados como locais de risco utilizando como parâmetro a taxa de incidência nos três últimos anos analisados maiores do que a média nacional e pertencentes ao grupos 2 (tendência crescente). Essa tabela possui uma coluna definida como Regra que indica em quais regras cada município se enquadra. Nessa tabela há a classificação de prioridade (aba Prioritários) de cada município, categorizado como S para Sim e N para Não, segundo o Plano Nacional de Controle de Tuberculose ([dVeS01]). A versão completa dessa tabela está disponível no diretório online do Instituto de Matemática e Estatística da Universidade de São Paulo ([San20]). 


\begin{tabular}{|c|c|c|c|c|c|c|}
\hline ID_Mun & Nom_Mun & MI & Grupos & Risco & Prioritários & Regra \\
\hline 3504701 & "BALBINOS & 871,41 & 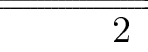 & $\overline{\mathrm{X}}$ & $\overline{\mathrm{N}}$ & 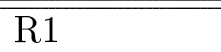 \\
\hline 3526506 & LAVINIA & 713,58 & 2 & $\mathrm{X}$ & $\mathrm{N}$ & $\mathrm{R} 1, \mathrm{R} 2, \mathrm{R} 7$ \\
\hline 3540853 & PRACINHA & 605,02 & 2 & $\mathrm{X}$ & $\mathrm{N}$ & $\mathrm{R} 1$ \\
\hline 3501400 & ALVARO DE CARVALHO & 525,23 & 2 & $\mathrm{X}$ & $\mathrm{N}$ & $\mathrm{R} 1$ \\
\hline 3528700 & MARABA PAULISTA & 495,43 & 2 & $\mathrm{X}$ & $\mathrm{N}$ & $\mathrm{R} 1, \mathrm{R} 2$ \\
\hline 3534906 & PACAEMBU & 477,40 & 2 & $\mathrm{X}$ & $\mathrm{N}$ & $\mathrm{R} 1, \mathrm{R} 2, \mathrm{R} 7$ \\
\hline 3541208 & PRESIDENTE BERNARDES & 393,23 & 2 & $\mathrm{X}$ & $\mathrm{N}$ & \\
\hline 3555109 & TUPI PAULISTA & 301,29 & 2 & $\mathrm{X}$ & $\mathrm{N}$ & $\mathrm{R} 1, \mathrm{R} 2$ \\
\hline 3521606 & IRAPURU & 300,28 & 2 & $\mathrm{X}$ & $\mathrm{N}$ & $\mathrm{R} 1, \mathrm{R} 2, \mathrm{R} 7$ \\
\hline 3517000 & GETULINA & 250,18 & 2 & $\mathrm{X}$ & $\mathrm{N}$ & \\
\hline 3551405 & SERRA AZUL & 249,45 & 2 & $\mathrm{X}$ & $\mathrm{N}$ & \\
\hline 3540754 & POTIM & 244,62 & 2 & $\mathrm{X}$ & $\mathrm{N}$ & $\mathrm{R} 7, \mathrm{R} 8$ \\
\hline 3542503 & REGINOPOLIS & 244,28 & 2 & $\mathrm{X}$ & $\mathrm{N}$ & $\mathrm{R} 1$ \\
\hline 3538907 & PIRAJUI & 238,15 & 2 & $\mathrm{X}$ & $\mathrm{N}$ & $\mathrm{R} 1$ \\
\hline 3516002 & FLORIDA PAULISTA & 211,79 & 2 & $\mathrm{X}$ & $\mathrm{N}$ & $\mathrm{R} 1, \mathrm{R} 2, \mathrm{R} 7$ \\
\hline 3526001 & JUNQUEIROPOLIS & 202,58 & 2 & $\mathrm{X}$ & $\mathrm{N}$ & $\mathrm{R} 1$ \\
\hline 3518503 & GUAREI & 201,82 & 2 & $\mathrm{X}$ & $\mathrm{N}$ & $\mathrm{R} 7, \mathrm{R} 8$ \\
\hline 3530102 & MIRANDOPOLIS & 196,36 & 2 & $\mathrm{X}$ & $\mathrm{N}$ & $\mathrm{R} 1, \mathrm{R} 2, \mathrm{R} 7$ \\
\hline 3544202 & RIOLANDIA & 187,20 & 2 & $\mathrm{X}$ & $\mathrm{N}$ & \\
\hline 3529203 & MARTINOPOLIS & 184,69 & 2 & $\mathrm{X}$ & $\mathrm{N}$ & $\mathrm{R} 1, \mathrm{R} 2$ \\
\hline 3510302 & CAPELA DO ALTO & 178,79 & 2 & $\mathrm{X}$ & $\mathrm{N}$ & $\mathrm{R} 7, \mathrm{R} 8$ \\
\hline 3506300 & BERNARDINO DE CAMPOS & 170,07 & 2 & $\mathrm{X}$ & $\mathrm{N}$ & \\
\hline 3511409 & CERQUEIRA CESAR & 168,79 & 2 & $\mathrm{X}$ & $\mathrm{N}$ & $\mathrm{R} 7$ \\
\hline 3527405 & LUCELIA & 168,70 & 2 & $\mathrm{X}$ & $\mathrm{N}$ & $\mathrm{R} 1, \mathrm{R} 2$ \\
\hline 3554805 & TREMEMBE & 164,86 & 2 & $\mathrm{X}$ & $\mathrm{N}$ & $\mathrm{R} 7, \mathrm{R} 8$ \\
\hline 3523602 & ITIRAPINA & 163,24 & 2 & $\mathrm{X}$ & $\mathrm{N}$ & \\
\hline 3556305 & VALPARAISO & 162,63 & 2 & $\mathrm{X}$ & $\mathrm{N}$ & $\mathrm{R} 2, \mathrm{R} 7$ \\
\hline 3509106 & CAIUA & 130,32 & 2 & $\mathrm{X}$ & $\mathrm{N}$ & $\mathrm{R} 1, \mathrm{R} 2$ \\
\hline 3531100 & MONGAGUA & 126,51 & 1 & $\mathrm{X}$ & $\mathrm{N}$ & $\mathrm{R} 7, \mathrm{R} 9, \mathrm{R} 10$ \\
\hline 3551009 & SAO VICENTE & 121,20 & 2 & $\mathrm{X}$ & S & $\mathrm{R} 7, \mathrm{R} 10$ \\
\hline 3518701 & GUARUJA & 118,35 & 1 & $\mathrm{X}$ & S & $\mathrm{R} 7, \mathrm{R} 10$ \\
\hline 3504404 & AVANHANDAVA & 115,40 & 2 & $\mathrm{X}$ & $\mathrm{N}$ & $\mathrm{R} 7$ \\
\hline 3521002 & IPERO & 115,35 & 2 & $\mathrm{X}$ & $\mathrm{N}$ & $\mathrm{R} 2, \mathrm{R} 7, \mathrm{R} 9$ \\
\hline 3553807 & TAQUARITUBA & 110,57 & 2 & $\mathrm{X}$ & $\mathrm{N}$ & $\mathrm{R} 7$ \\
\hline 3537602 & PERUIBE & 93,38 & 1 & $\mathrm{X}$ & S & \\
\hline 3516408 & FRANCO DA ROCHA & 89,81 & 1 & $\mathrm{X}$ & S & $\mathrm{R} 7, \mathrm{R} 8$ \\
\hline 3522109 & ITANHAEM & 87,97 & 2 & $\mathrm{X}$ & S & $\mathrm{R} 2, \mathrm{R} 9, \mathrm{R} 10$ \\
\hline 3536208 & PARIQUERA-ACU & 87,32 & 1 & $\mathrm{X}$ & $\mathrm{N}$ & $\mathrm{R} 7, \mathrm{R} 9$ \\
\hline 3534609 & OSVALDO CRUZ & 86,92 & 2 & $\mathrm{X}$ & $\mathrm{N}$ & \\
\hline 3529658 & MESOPOLIS & 86,42 & 2 & $\mathrm{X}$ & $\mathrm{N}$ & \\
\hline
\end{tabular}

Tabela 4.4: 40 municípios considerados de risco de tuberculose, após aplicar um limiar de 40 casos por 100 mil hab. para a variável MI e considerar o grupo 2 de tendência. A coluna Prioritários indica $S$ para municípios que foram considerados como prioritários, conforme o PNCT e $N$ caso contrário. Os locais de riscos foram assinalados com $X$. A coluna Regra indica em quais regras esses municípios se enquadram. 


\subsection{Discussão}

Com base nas análises descritas anteriormente, o padrão identificado pela distribuição espacial da média da taxa de incidência nos três últimos anos indica que há maior risco de tuberculose nas regiões da costa litorânea, próximas à capital do estado e próximas de Presidente Prudente, Adamantina e Araçatuba.

Classificamos como municípios de risco aqueles que possuem incidência crescente e o mesmo ocorre em seus vizinhos (regra R1) ou que possuem, isoladamente, incidência crescente e maior que 40 casos por 100 mil habitantes. Os municípios inclusos nesta categoria possuem incidência média nos últimos três anos estudados de 303,66 casos por 100 mil hab. (95\%, [193,52; 413,80]).

O comportamento da série temporal em Campos de Jordão pode ser explicado devido a decentralização dos casos de tuberculose, pois até o início do século os sanatórios nessa localidade concentravam muitos casos de tuberculose ([CdSK02], [Cai19]).

O número de casos brutos registrados em Borá no ano de 2005 trouxeram alguns questionamentos sobre o comportamento da doença nessa localidade e ano. Entretanto, é provável que esse número tenha sido gerado por um erro de digitação ou organização dos dados no Sistema Único de Saúde. Uma análise mais detalhada para investigar o comportamento aberrante nessa localidade é necessário e não foi o objetivo deste trabalho.

Não há dúvidas que a faixa etária mais atingida são os idosos e adultos. Esse padrão foi encontrado pela metodologia adotada neste trabalho (regras R7, R8 e Tabela 4.3) e confirmada com os padrões encontrados nas literaturas ([dVeS14] e [PdAME $\left.\left.{ }^{+} 15\right]\right)$. Há estudos que indicam que essas mesmas faixas etárias predominam na taxa de mortalidade por tuberculose ([Per07]). A despesa com saúde e assistência básica são os fatores socioeconômicos que mais influenciam esta variável e devem ser monitoradas pelos órgãos de controle.

A relação entre densidade de moradores por dormitório maiores do que 2, IDHM e a incidência de tuberculose tem sido estudada por alguns autores ([dLSGSVG ${ }^{+}$07], [adSdSP09], [Siq14] e $\left.\left[\mathrm{PdAME}^{+} 15\right]\right)$. Os padrões obtidos pela metodologia proposta confirmaram essa conjectura, por meio das regras R9 e R10. Estar no mesmo dormitório que uma pessoa infectada aumenta o risco de adquirir a doença na forma latente.

A dependência da taxa de incidência com a distância ao litoral ou presídio destacou-se durante a mineração dos dados (R1, R3, R4, R5, R11 e padrões espaciais). Locais próximos a costa litorânea e com altas taxas de incidência de tuberculose também foram encontrados por Antonio L. Rodrigues Jr. et al. (2006). Neste artigo, o autor discute que o fluxo migratório e o escoamento em direção ao litoral e exterior possa ser responsável pela altas taxas de coinfecção de tuberculose e AIDS ([JNdC06]). Pessoas convivendo em locais próximos a presídios também implicam em maior risco de tuberculose, visto que a condição de saúde das pessoas privadas de liberdade é extremamente precária. Em 2016, o estado de São Paulo apresentou cerca de 14,3\% dos casos de TB associado às pessoas privadas de liberdade ([Min18]). 
Arakawa et al. (2017), indicou que os municípios considerados não prioritários pelo PNCT, localizados no interior de São Paulo e em cidade de pequeno porte, possuem desempenho insatisfatório em relação ao programa de combate à tuberculose ([AMdPA+17]). Esse padrão também foi identificado com o uso da metodologia adotada neste trabalho e os municípios indicados como locais de risco concordaram com esta literatura.

Há municípios que foram considerados como prioritários pelo PNCT em 2004 ([dVeS01]), porém não foram categorizados como locais de risco, pois não há um consenso quanto ao limiar considerado alarmante, referente a incidência de tuberculose. Pelo mesmo argumento, é preciso considerar que áreas de baixo risco podem ser resultado de subnotificações, sendo necessário aprimorar a coleta e a qualidade dos dados ([AYPZ $\left.\left.{ }^{+} 17\right]\right)$. Não cabe, neste trabalho, a discussão dos parâmetros definitivos da doença.

Os resultados aqui obtidos por meio do encadeamento de diversas técnicas de tratamento e mineração de dados, demostraram concordância os conhecimentos do comportamento da tuberculose e sua forma de espalhamento. No entanto, por meio da análise espacial mais detalhada, municípios que não pertencem à lista de prioritários nos órgãos de saúde, possuem tendência crescente da taxa de incidência.

A base de dados foi escolhida levando em consideração a regularidade, qualidade e quantidade dos dados disponíveis. Mas não há nenhuma restrição para inclusão ou substituição de dados. Desde que as etapas de tratamento e consolidação sejam realizadas, a metodologia pode ser utilizada ou mesmo aprimorada com novas regras. 


\section{Capítulo 5}

\section{Conclusões}

\subsection{Considerações Finais}

A tuberculose é uma doença perigosa e que está longe de ser erradicada. Diversas estratégias foram propostas para sua erradicação desde o lançamento do PNCT. Porém, existem localidades e fatores socioeconômicos que impedem o sucesso dessas ações.

Fatores socioeconômicos estão fortemente relacionados com a tuberculose e sua transmissão. Por essa razão, este trabalho procurou identificar padrões entre a doença e os dados socioeconômicos por meio da mineração de dados e análise de dados espaciais.

Uma metodologia foi aplicada para obter as regras e padrões espaciais associando a doença e os atributos socioeconômicos, identificando locais prioritários e com maiores riscos. Relações com a incidência e a tendência de tuberculose também foi identificada através da metodologia proposta.

Todos os objetivos estabelecidos foram alcançados e a principal contribuição deste trabalho é a metodologia proposta para a solução do problema, sendo totalmente flexível (base de dados, a doença e as regras extraídas), que permite identificar correlações entre a doença e os dados socioeconômicos, sendo passíveis de monitoramento ou ser usada como alerta de controle em regiões do estado.

Os principais desafios encontrados durante o tratamento dos dados foram a obtenção de dados consistentes que pudessem ser minerados. Nessa etapa, a escolha da doença não é tão importante, mas sim, a escolha de dados concisos e limpos. Dados faltantes, municípios em duplicidades e sem identificação são fatores que foram encontrados constantemente na análise dos dados.

\subsection{Sugestões para Pesquisas Futuras}

A utilização de setores censitários pode identificar regras diferentes daquelas encontradas neste trabalho, permitindo uma busca mais refinada.

Usar uma base de dados multirrelacional, pois permite concatenar bases de outras fontes e espécies, por exemplo, dados não estruturados. 
Implementação de uma técnica temporal para verificar a mudança das regras ao longo do tempo. Uma proposta seria usar um algoritmo de regras de associação espaço temporal para obter padrões que permitem prever lugares com maiores riscos de tuberculose nos próximos anos.

Criar um índice socioeconômico espaço-temporal, onde valores acima de um limiar tem maior risco da doença nos próximos anos é uma sugestão para trabalhos futuros, pois permite utilizar parte da metodologia como forma de caracterizar esse índice. 


\section{Referências Bibliográficas}

[adSdSP09] Michael Guimar aes de Souza e Eduardo da Silva Pinheiro. Incidência e Distribuição da Tuberculose na cidade de Manaus. Revista Geográfica Acadêmica, $3(1): 36-43,2009.88$

[Ale07] Aretha Barbosa Alencar. Mineração e visualização de coleções de séries temporais. Dissertação de Mestrado, Universidade de São Paulo - São Carlos - ICMC, Brasil, Novembro 2007. 19

[Alm12] Eduardo Almeida. Econometria Espacial Aplicada. Alinea, Campinas, BR, 2012. 32

[Alp04] Ethem Alpaydin. Introduction to Machine Learning. The MIT Press, 2004. 15, 21,65

[AMdPA $\left.{ }^{+} 17\right]$ Tiemi Arakawa, Gabriela Tavares Magnabosco, Rubia Laine de Paula Andrade, Maria Eugenia Firmino Brunello, Aline Aparecida Monroe, Antonio Ruffino Netto, Lucia Marina Scatena e Tereza Cristina Scatena Villa. Programa de controle da tuberculose no contexto municipal: avaliação de desempenho. Revista de Saúde Pública, 51:1-9, 2017. 89

[Ans95] Luc Anselin. Local indicators of spatial association lisa. Geographical Analysis, $27(2): 93-115,1995.35$

[AS95] Rakesh Agrawal e Ramakrishnan Srikant. Mining Generalized Association Rules. Em VLDB '95 Proceedings of the 21th International Conference on Very Large Data Bases, San Francisco, CA, USA, páginas 407-419, 1995. 24

[ASI93] Rakesh Agrawal, Arun Swami e Tomasz Imielinski. Mining association rules between sets of items in large databases. Em Proceedings of the 1993 ACM SIGMOD International Conference on Management of Data, Washington, DC, USA, volume 22, páginas 207-216, 1993. 24

[ASI94] Rakesh Agrawal, Arun Swami e Tomasz Imielinski. Fast Algorithms for Mining Association Rules. Em Proceedings of the 1994 ACM SIGMOD International Conference on Management of Data, Washington, DC, USA, páginas 487-499, 1994. 24,26

[Ass12] Fernando Assunção. Estratégias para tratamento de variáveis com dados faltantes durante o desenvolvimento de modelos preditivos. Dissertação de Mestrado, Instituto de de Matemática e Estatística da USP, Brasil, Junho 2012. 12

[ASW15] Saeed Aghabozorgi, Ali Seyed Shirkhorshidi e Teh Ying Wah. Time-Series clustering - A decade review. Information Systems, 53:16 - 38, 2015. 62

[AYPZ ${ }^{+}$17] Luiz Henrique Arroyo, Mellina Yamamura, Simone Terezinha Protti-Zanatta, Alcione Pereira Biffi Fusco, Pedro Fredemir Palha, Antônio Carlos Vieira Ramos, Severina Alice Uchoa e Ricardo Alexandre Arc $\widetilde{A}^{a}$ ncio. Identificação de áreas de 
risco para a transmissão da tuberculose no município de São Carlos, São Paulo, 2008 a 2013. Epidemiologia e Serviço de Saúde, 26(3):525 - 534, 2017. 89

[Bar12] David Barber. Bayesian Reasoning and Machine Learning. Cambridge, 2012. 15

[Boa16] Global TB Community Advisory Board. Extrapulmonary tb. http://www. tbonline.info/posts/2016/3/31/extrapulmonary-tb/, 2016. Último acesso em 09/12/2018. 3

[BPGR08] Roger S. Bivand, Edzer J. Pebesma e Virgilio Gomez-Rubio. Applied Spatial Data Analysis wich R. Springer, New York, NY, 2008. 32, 37, 56

[BRA90] BRASIL. Estatuto da criança e do adolescente: Lei No 8069, de 13 de julho de 1990. Imprensa Oficial, 1990. 41

[BRA03] BRASIL. Estatuto do idoso: Lei No 10.741, de 1 de outubro de 2003. Imprensa Oficial, 2003. 41

[Cai19] Luiza Caires. Campos do Jordão epidemia de tuberculose deu origem à cidade sanatório que hoje é destino turístico. https://jornal.usp.br/ciencias/ ciencias-humanas/, 2019. Último acesso em 09/01/2020. 88

[Can12] Marcos Daniel Cano. Mineração de regras de associação sequenciais em séries temporais e visualização: Aplicação em dados agrometeorológicos. Dissertação de Mestrado, Instituto de Computação, Universidade de São Carlos, Brasil, 2012. 30

[Car06] Janice Haney Carr. Centers for Disease Control and Prevention. https://phil. cdc.gov/Details.aspx?pid=9997, 2006. Último acesso em 09/12/2018. xiii, 2

[CdS09] Cassio Oliveira Camilo e Joao Carlos da Silva. Mineração de dados: Conceitos, Tarefas, Métodos e Ferramentas. Relatório técnico, Universidade Federal de Goiás, Agosto 2009. 10

[CdSK02] Marcus B. Conde, Gilvan Muzy de Souza e Afrânio L. Kritski. Tuberculose sem medo. Atheneu, 1 edição, 2002. 3, 4, 7, 88

[CN07] Ahlame Douzal Chouakria e Panduranga Naidu Nagabhushan. Adaptive dissimilarity index for measuring time series proximity. Advances in Data Analysis and Classification, 1:5 - 21, 2007. 19

[CVZH97] Peter Cabena, Jaap Verhees, Alessandro Zanasi e Pablo Hadjnian. Discovering Data Mining: From Concept to Implementation. Prentice Hall, 1 edição, 1997. 10

[dAaP18] Secretaria de Administração Penitenciária. Unidades Prisionáis. http://www. sap.sp.gov.br/, 2018. Último acesso em 15/10/2018. 43

[Dan06] Thomas M. Daniel. The history of tuberculosis. Respiratory Medicine, 100(11):1862-1870, 2006. 3

[DAT18] DATASUS. Tuberculose - Casos Confirmados Notificados no Sistema de Informação de Agravos de Notificação - Brasil. http://tabnet.datasus.gov.br/cgi/tabcgi. exe?sinannet/cnv/tubercbr.def, 2018. Último acesso em 11/05/2018. 39, 40, 67

[DD16] Michel Marie Deza e Elena Deza. Encyclopedia of Distances. Springer, 4 edição, 2016. 19 
[dDHnB10] Atlas do Desenvolvimento Humano no Brasil. Ranking - Todo o Brasil. http: //www.atlasbrasil.org.br/2013/ranking, 2010. Último acesso em 20/09/2018. 42, 57

[dGeEI19] Instituto de Geografia e Estatística (IBGE). Estimativa da População. https://www.ibge.gov.br/estatisticas-novoportal/sociais/populacao/ 9103-estimativas-de-populacao.html? =\&t=downloads, 2019. Último acesso em 26/02/2020. 45

[DLR77] Arthur Pentland Dempster, Nan McKenzie Laird e Donald Bruce Rubin. Maximum likelihood from incomplete data via the em algorithm. Journal of the Royal Statistical Society, 39(1B):1 - 38, 1977. 13, 47

[dLSGSVG ${ }^{+}$07] Maria de Lourdes Sperli Geraldes Santos, Silvia Helena Figueiredo Vendramini, Claudia Eli Gazetta, Sonia Aparecida Cruz Oliveira e Tereza Cristina Scatena Villa. Poverty: Socioeconomic Characterization at Tuberculosis. Revista Latino Americana Enfermagem, 15:762 - 767, 2007. 88

[dMdSaP17] Santa Casa de Misericórdia de São Paulo. Hospital São Luiz Gonzaga. $\quad$ http://www.santacasasp.org.br/portal/site/complexo/hospitais/ hospital-sao-luiz-gonzaga, 2017. Último acesso em 10/06/2018. 4

[dMP18] Rodrigo Fernandes de Mello e Moacir Antonelli Ponti. Machine Learning - A Pratical Approach on the Statistical Learning Theory. Springer, Brasil, SP, 2018. 15

[dNadAdNaS18] Sistema de Notificação de Agravos de Notificação (SINAN). Tuberculose. http: //portalsinan.saude.gov.br/tuberculose, 2018. Último acesso em 24/07/2018. 40

[dPeTR1] Rede Brasileira de Pesquisa em Tuberculose REDETB. A História da Tuberculose. http://www.redetb.org/index.php/sobre-a-tuberculose/ a-historia-da-tuberculose, 21-. Último acesso em 20/01/2019. 2

[dS63] Itazil Benício dos Santos. Vida e obra de Manoel de Abreu: O criador da Abreugrafia. Irmãos Pongetti, 1 edição, 1963. 4

[dS16] Pedro Leal Pazzini da Silva. Um Estudo sobre o Agrupamento de Séries Temporais e sua Aplicação em Curvas de Carga Residenciais. Dissertação de Mestrado, Universidade Federal de Minas Gerais, Brasil, 2016. 19

[dSdEdSaP1 ] Secretaria da Saúde do Estado de São Paulo. Tuberculose - Tratamento. http:// www.saude.sp.gov.br/ses/perfil/cidadao/temas-de-saude/tuberculose, 21-? Último acesso em 05/04/2018. 3, 5

[dSJ04] Jarbas Barbosa da Silva Jr. Tuberculose - Guia de Vigilância Epidemiológica. Jornal Brasileiro de Pneumologia, 30(1):57-76, 2004. 3

[dSPB16] Leandro Augusto da Silva, Sarajane Marques Peres e Clodis Boscarioli. Introdução à Mineração de Dados - Com Aplicações em R. Elsevier, 3 edição, 2016. 10, $14,15,21,65$

[dVeS01] Secretaria de Vigilância em Saúde. Programa Nacional de Controle da Tuberculose, 2001. OMG Document 02-06-33. 5, 86, 89

[dVeS06] Secretaria de Vigilância em Saúde. Brasil Livre da Tuberculose - Plano Nacional pelo Fim da Tuberculose como Problema de Saúde Pública no Brasil. Ministério da Saúde, 2 edição, 2006. 5 
[dVeS14] Secretaria de Vigilância em Saúde. O Controle da Tuberculose no Brasil Avanços, inovações e desafios, 2014. 88

[dVeS18] Secretaria de Vigilância em Saúde. Implantação do Plano Nacional pelo Fim da Tuberculose como Problema de Saúde Pública no Brasil: primeiros passos rumo ao alcance das metas. Boletim Epidemiológico, 49(11):1-18, 2018. 5

[eFJR62] Robert Reuven Sokal e F. James Rohlff. The Comparion of Dendograms by Objective Methods. Taxon, 2(11):33-40, 1962. 23

[ELLS11] Brian S. Everitt, Sabine Landau, Morven Leese e Daniel Stahl. Cluster Analysis. Wiley, 5 edição, 2011. 17, 21, 23, 24

[Fil01] Claudio Bertolli Filho. História Social da Tuberculose e do Tuberculoso 19901950. FIOCRUZ, 2001. 3

[FPSS96] Usama Fayyad, Gregory Piatetsky-Shapiro e Padhraic Smyth. From data mining to knowledge discovery in databases. AI Magazine, 17(3):37-54, 1996. xiii, 9

[GC39] João Grieco e Francisco A. Cardoso. A tuberculose em São Paulo. Em Primeiro Congresso Nacional de Tuberculose, páginas 305-329. Revista Paulista de Tisiologia, 1939. 4

[Gon05] Eduardo Corrêa Gonçalves. Regras de Associação e suas Medidas de Interesse Objetivas e Subjetivas. Relatório técnico, Universidade Federal Fluminense, Março 2005. 30

[Gua11] Adalgiza Rosemara Guarnier. Fatores associados ao desfecho dos casos novos de tuberculose no estado de São Paulo em 2008. Dissertação de Mestrado, Faculdade de Ciências Médicas da Santa Casa de São Paulo, Brasil, 2011. 5

[HGDASTMK06] Van Der Heijden GL, Donders AR, Stijnen T e Moons KG. Imputation of missing values is superior to complete case analysis and the missing-indicator method in multivariable diagnostic research: a clinical example. Journal of Clinical Epidemiology, 59(10):1102-1109, 2006. 13

[HGTP07] Miguel Aiub Hijjar, Germano Gerhardt, Gilmário M. Teixeira e Maria José Procópio. Retrospecto do controle da tuberculose no Brasil. Revista de Saúde Pública, $41(1): 50-58,2007.4,5$

[HK06] Jiawei Han e Micheline Kamber. Data Mining: Concepts and Techniques. Morgan Kaufmann, 2 edição, 2006. 10, 12, 13, 14, 21, 24, 47, 57, 65

[HKB11] James Honaker, Gary King e Matthew Blackwell. AMELIA ii: A Program for Missing Data. Journal of Statistical Software, 45(7):1 - 47, 2011. xiii, 14, 49

[HTF01] Trevor Hastie, Robert Tibshirani e Jerome Friedman. The Elements of Statistical Learning. Springer, 2 edição, 2001. 15, 24, 65

[Ize08] Alan Julian Izenman. Modern Multivariate Statistical Techniques: Regression, Classification, and Manifold Learning. Springer Texts in Statistics, 2008. xvii, $15,21,22,23$

[JBBA09] Joseph F. Hair Jr., Willian C. Black, Barry J. Babin e Rolph E. Anderson. Multivariate Data Analysis. Prentice Hall, 7 edição, 2009. 12, 14

[JNdC06] Antonio L. Rodrigues Jr., Antonio Ruffino Netto e Euclides Ayres de Castilho. Distribuição espacial da co-infecção M. tuberculosis/HIV no estado de São Paulo em 1991 a 2001. Revista de Saúde Pública, 40(2):265-270, 2006. 88 
[KR05] Yun Sing Koh e Nathan Rountree. Finding Sporadic Rules Using Apriori-inverse. Em PAKDD '05 Proceedings of the 9th Pacific-Asia conference on Advances in Knowledge Discovery and Data Mining, Hanoi, Vietnam, páginas 97-106, 2005. 30

[Leo00] Carla Leonel. Medicina: Mitos e Verdades. Saúde e Vida, 4 edição, 2000. 2

[LHM99] Bing Liu, Wynne Hsu e Yiming Ma. Mining Association Rules with Multiple Minimum Supports. Em SIGKDD '99 Proceedings of the 2000 ACM SIGMOD international conference on Knowledge discovery and data mining, San Diego, California, USA, páginas 337-341, 1999. 30

[Liu68] C. L. Liu. Introduction to Combinatorial Mathematics. McGraw Hill, 1968. 16

[LL14] Daniel T. Larose e Chantal D. Larose. Discovering Knowledge in Data - An Introduction to Data Mining. Wiley, 2 edição, 2014. 14, 57, 65

[LnFR17] Antonio José Rocha Luzardo, Rafael March Casta neda Filho e Igor Brum Rubim. Análise Espacial Exploratória com o Emprego do Índice de Moran. GEOgraphia, 19(40):162 - 179, 2017. 34, 36

[Mac99] Juceli Maria Maciel. Microbiologia e Parasitologia. Ulbra, 2 edição, 1999. 2

[Mad04] M. Monir Madkour. Tuberculosis. Springer, 1 edição, 2004. xiii, 1, 2, 3

[McC07] Colleen McCue. Data Mining and Predictive Analysis - Intelligence Gathering and Crime Analysis. Elsevier, 2007. 11, 15

[Met06] Jean Metz. Interpretação de clusters gerados por algoritmos de clustering hierárquico. Dissertação de Mestrado, Universidade de São Paulo - São Carlos, Brasil, 2006. 24

[Min18] Ministério da Saúde. Secretaria de Vigilância em Saúde - Departamento de Vigilância das Doenças Transmissíveis, Brasília, DF. Panorama da tuberculose no Brasil: Diagóstico situacional a partir de indicadores epidemiológicos e operacionais, 2018. 88

[Mor50] Patrick Alfred Pierce Moran. Notes on continuous stochastic phenomena. Biometrika, 37:17-23, 1950. 32

[MSH15] John F. Murray, Dean E. Scharaufnagel e Philip C. Hopewell. Treatment of tuberculosis: A historical perspective. ATS Discoveries Series, 12(12):1749 1759, 2015. 3

[Mur04] John F. Murray. A Century of Tuberculosis. American Journal of Respiratory and Critical Care Medicine, 169(11):1181-1186, 2004. 1

[MV14] Pablo Monteiro e José A. Vilar. An R Package for Time Series Clustering. Journal of Statistical Software, 62(1):1 - 43, 2014. 20, 62

[Nun07] Luciana Neves Nunes. Métodos de Imputação de Dados Aplicados na Área da Saúde. Tese de Doutorado, Universidade Federal do Rio Grande do Sul, Faculdade de Medicina, Curso de Epidemiologia., Porto Alegre, 2007. 13

[Org18a] World Health Organization. Global Tuberculosis Report 2018. CC BY-NCSA 3.0 IGO, 2018. xiii, 2, 7, 8

[Org18b] World Health Organization. Tuberculosis. http://www.who.int/en/news-room/ fact-sheets/detail/tuberculosis, 2018. Último acesso em 09/05/2018. 1, 2 
[Pau18] Cidades Paulistas. Litoral Paulista. http://www.cidadespaulistas.com.br/prt/ cnt/mp-litoral.htm, 2018. Último acesso em 15/10/2018. 43

[PdAME ${ }^{+}$15] Alessandra Gonçalves Lisbôa Pereira, Roberto de Andrade Medronho, Claudia Caminha Escosteguy, Luis Iván Ortiz Valencia e Mônica de Avelar Figueiredo Mafra Magalhães. Distribuição espacial e contexto socioeconômico da tuberculose, Rio de Janeiro, Brasil. Revista de Saúde Pública, 49:2 - 9, 2015. 36, 88

[Per07] Edméa Costa Pereira. Mortalidade relacionada a tuberculose no Município de São Paulo 2002 a 2004. Tese de Doutorado, Universidade de São Paulo, Faculdade de Saúde Pública, Curso de Saúde Pública., São Paulo, 2007. 88

[Piv06] Marina Abichabki Pivato. Mineração de Regras de Associação em Dados Georreferenciados. Dissertação de Mestrado, Instituto de Ciências Matemáticas e de Computação, Brasil, 2006. 24

[PS91] G. Piatetsky-Shapiro. Discovery, analysis and presentation of strong rules. Em G. Piatetsky-Shapiro e W. J. Frawley, editors, Knowledge Discovery in Databases, páginas 229-248. AAAI Press, 1991. 31

[Roe18] Pjotr Roelofsen. Time series clustering. Dissertação de Mestrado, Vrije Universiteit Amsterdam, Março 2018. 62

[Rou87] Peter J. Rousseeuw. Silhouettes: a Graphical aid to the Interpretation an Validation of Cluster Analysis. Journal of Computational and Applied Mathematics, 20:53-65, 1987. 23

[Rub76] Donald B. Rubin. Inference and Missing Data. Biometrika, 63(3):581 - 592, 1976. 12,47

[Rub87] Donald B. Rubin. Multiple Imputation for Nonresponse in Surveys. Wiley series in probability and mathematical statistics, 1 edição, 1987. 13, 47, 49

[San03] Marcelo Kaminski Sanches. Aprendizado de máquinas semi-supervisionado: proposta de um algoritmo para rotular exemplos a partir de poucos exemplos rotulados. Dissertação de Mestrado, Instituto de Ciências Matemáticas e de Computação, Brasil, Junho 2003. 15

[San20] Erick Pereira Santos. Bases de Dados. https://www.ime.usp.br/ erickps/ RESULTADOS, 2020. Último acesso em 08/01/2020. 86

[Sau18] Tua Saude. Vacina BCG: para que serve e quando tomar. http://www.tuasaude. com/vacina-contra-tuberculose-bcg, 2018. Último acesso em 16/05/2018. 3

[SBW44] Albert Schatz, Elizabeth Bugle e Selman Abraham Waksman. Streptomycin, a substance exhibiting antibiotic activity against gram-positive and gram-negative bacteria. Proceedings of the Society for Experimental Biology and Medicine, 55(1):66-69, 1944. 3

[SdSdEdP1] Secretaria da Saúde do Estado do Paraná. Tuberculose. http://www.saude.pr. gov.br/modules/conteudo/conteudo.php?conteudo=939/, 21-. Último acesso em 05/04/2018. 3,5

[SEA18] SEADE. Base de dados do SEADE - Sistema Estadual de Análise de Dados. http://www.seade.gov.br/, 2018. Último acesso em 13/01/2018. 42 
[Siq14] Alexandre San Pedro Siqueira. Determinantes socioeconômicos da produção da tuberculose Um estudo no municipio de Itaborai, Região Metropolitana do Rio de Janeiro, no periodo de 2000 a 2011. Tese de Doutorado, Fundação Osvaldo Cruz, Escola Nacional de Saúde Pública., Rio de Janeiro, 2014. 36, 88

[Smi05] Dr. Scott Smith. Isoniazid. https://web.stanford.edu/class/humbio103/ ParaSites2005/Isoniazid/history.html, 2005. Último acesso em 11/05/2018. 3

[SSBD14] Shai Shalev-Shwartz e Shai Ben-David. Understanding Machine Learning from theory to algorithms. Cambridge, 2014. 15

[TKS04] Pang-Ning Tan, Vipin Kumar e Jaideep Srivastava. Selecting the Right Interestingness Measure for Association Patterns. Information Systems, 4:293 - 313, 2004. 30

[TSK06] Pang-Ning Tan, Michael Steinbach e Vipin Kumar. Introduction to Data Mining. Person Education, 2 edição, 2006. xiii, 10, 14, 17, 18, 24, 26, 27, 57, 65

[Ven19] Chris Veness. Calculate distance, bearing and more between latitude and longitude points. https://www.movable-type.co.uk/scripts/latlong.html, 2019. Último acesso em 08/04/2019. 43

[Wik18a] Wikipedia. Formule de Haversine. https://https://fr.wikipedia.org/wiki/ Formule_de_haversine, 2018. Último acesso em 08/04/2019. 43

[Wik18b] Wikipedia. Tuberculosis. https://en.wikipedia.org/wiki/Tuberculosis/, 2018. Último acesso em 10/05/2018. 2

[Wou10] Henry Wouk. Tuberculosis. Marshall Cavendish Benchmark, 1 edição, 2010. xiii, $1,2,3,4$

[Wu70] Chien-Fu Jeff Wu. On the convergence properties of the em algorithm. The Annals of Statistics, 11:95 - 103, 1970. 14

[XW09] Rui Xy e Donald C. Wunsch. Clustering. Wiley, 2 edição, 2009. xiii, 16, 17, 19, $21,22,23$

[Zak01] Mohammed J. Zaki. Spade: An efficient algorithm for mining frequent sequences. Machine Learning, 42:31-60, 2001. 29

[Zik18] Paul Zikopoulos. Unstructured Data Analytics. Wiley, 7 edição, 2018. 10 